\title{
The effect of Lewis acids on the intramolecular Diels-Alder reaction of the furan diene
}

\author{
Christine Rogers and Brian A. Keay' \\ Department of Chemistry, University of Calgary, Calgary, Alta., Canada T2N IN4 \\ Received May 15, 1992
}

\begin{abstract}
Christine Rogers and Brian A. Keay, Can. J. Chem. 70, 2929 (1992).
A general method is described for effecting the intramolecular Diels-Alder reaction of the furan diene in which the side arm connecting the diene to the dienophile contains four carbon atoms. The use of 1.1 equivalents of methylaluminum dichloride at $-78^{\circ} \mathrm{C}$ for $2-8 \mathrm{~h}$ shifts the Diels--Alder equilibrium towards the products and provides the oxatricyclo adducts in good to excellent yield. Catalytic quantities of methylaluminum dichloride ( 10 mol\%) provided a higher quantity of adduct than excess Lewis acid when the enone was substituted with alkyl groups. The scope was extended to include a precursor containing a five carbon atom side arm, and two examples containing acetylenic dienophiles that were activated by a carbonyl moiety on the side arm. Precursors having a four carbon atom side arm provided only oxatricyclo adducts having the side arm syn to the oxygen bridge. The assignment of the stereochemistry of the oxatricyclo adducts is discussed in detail.
\end{abstract}

Christine Rogers et Brian A. Keay. Can. J. Chem. 70, 2929 (1992).

On décrit une méthode générale d'effectuer une réaction intramoléculaire de Diels--Alder avec des diènes furaniques portant une chaîne latérale de quatre atomes de carbone qui relie le diène au diénophile. L'utilisation de 1,1 équivalent de dichlorure de méthylaluminium, à $-78^{\circ} \mathrm{C}$, pendant $2-8 \mathrm{~h}$, déplace l'equilibre de Diels-Alder vers les produits et les adduits oxatricyclo avec des rendements allant de bons à excellents. Lorsqu'on substitue l'énone avec des groupes alkyles, les quantités d'adduit formées lorsqu'on utilise des quantités catalytiques de dichlorure de méthylaluminium (10 mol\%) sont plus grandes que celles obtenues avec un excès d'acide de Lewis. On a étendu le champ d'application à un précurseur contenant une chaîne latérale de cinq atomes de carbone et à deux exemples contenant des diénophiles acétyléniques activés par une portion carbonyle sur la chaîne latérale. Les précurseurs portant une chaîne latérale contenant quatre atomes de carbone ne fournissent que les adduits oxatricyclo dans lesquels la chaîne latérale est syn par rapport au pont oxygène. On discute en détail de l'attribution de la stéréochimie des adduits oxatricyclo.

[Traduit par la rédaction]

The intramolecular Diels-Alder (IMDA) reaction is a widely utilized synthetic strategy for the simultaneous formation of two rings with high stereo- and regio-control (1). The use of a furan moiety as the diene component in the IMDA reaction leads to the creation of an oxygenated cyclohexane ring in a rigid cycloadduct that has potential application for the synthesis of natural products. The intramolecular Diels-Alder reaction of the furan diene (IMDAF) has been studied extensively both when the tether connecting the diene and dienophile consists of three carbon atoms $(1,2)$, and when the tether contains a heteroatom and is either three $(n=1)$ or four $(n=2)$ atoms in length (eq. 1) $(1,3)$. The IMDAF reaction of precursors that have a side arm containing four $(n=2)(1,4)$ or five $(n=3)(1,5)$ carbon atoms have been studied to a lesser extent, and are generally reported to have equilibria that lie toward starting material. Methods employed to overcome the unfavourable equilibrium have included heat (4a), $\beta$-cyclodextrin (6), aqueous solutions $(4 h, 7)$, substituted side arms $(3 g, 3 h, 4 k$, $4 m, 4 n, 5 b, 8)$ and high pressure $(4 b, 4 c, 4 j)$. The success of these methods has been variable. The first four methods have produced increased starting material:adduct (SM:A) ratios for precursors with unsubstituted dienophiles but usually required long reaction times (2-14 days). High pressure $(1.0-1.2 \mathrm{GPa})$ has been successful in overcoming the unfavourable equilibrium with both unsubstituted and substituted dienophiles; however, the isolated yields of the adducts have been poor, the reaction scale is limited, and access to specialized equipment is necessary. We therefore sought

\footnotetext{
${ }^{1}$ Author to whom correspondence may be addressed.
}

a generally applicable method for performing the IMDAF reaction that would overcome these limitations.
[1]

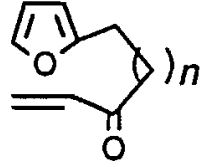

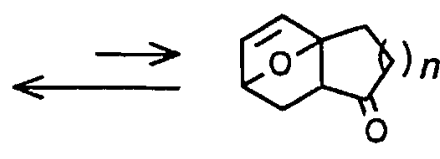

$n=1$ (a $\mathrm{CH}_{2}$ may be substituted by an $\mathrm{S}, \mathrm{O}$, or $\mathrm{N}$ atom) $n=2$ (a $\mathrm{CH}_{2}$ may be substituted by an $\mathrm{O}$ or $\mathrm{N}$ atom)

Lewis acids have been used extensively in the intermolecular Diels-Alder reaction of the furan diene (9) to increase both the regioselectivity and the rate of reaction. Lewis acids have also been used to accelerate the rate of the intramolecular Diels-Alder reaction (10); however, their successful use in the IMDAF reaction $(4 i, 5 a, 11)$ has been limited to only two reports: one employing an internally coordinated magnesium salt (12), and the second an application of zinc iodide (13). The paucity of examples of Lewis acid-mediated IMDAF reactions may be due to competing side reactions such as: (a) the polymerization of the furan ring or the dienophile (14), $(b)$ the susceptibility of the furan ring to Friedel-Crafts-type reactions (14), and (c) the aromatization of the oxatricyclo adducts (15). We recently reported that the IMDAF reaction, in which the side arm contained four or five carbon atoms, is accelerated by the Lewis acid methylaluminum dichloride at low temperatures to provide the oxatricyclo adducts in good to excellent yield (16). We herein provide a full account of this work.

\section{Preparation of IMDAF precursors 1-17}

Precursors 1-8 were prepared as outlined in Scheme 1. Lithiation of either furan 18 or 2-methylfuran 19 under 
<smiles>Pc1ccco1</smiles>

$18 \mathrm{R}=\mathrm{H}$ $19 \mathrm{R}=\mathrm{Me}$

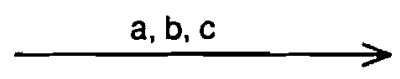

$\overbrace{\mathrm{R}}^{11} \mathrm{P}_{\mathrm{R}_{1}} \mathrm{R}_{\mathrm{R}_{2}}^{\mathrm{O}} \leftarrow$

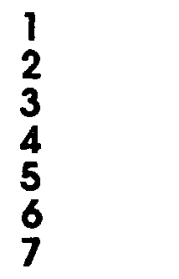

$\mathrm{R}=\mathrm{R}_{1}=\mathrm{R}_{2}=\mathrm{H}$ $\mathrm{R}=\mathrm{R}_{2}=\mathrm{H}, \mathrm{R}_{1}=\mathrm{Me}$ $\mathrm{R}=\mathrm{R}_{1}=\mathrm{H}, \mathrm{R}_{2}=\mathrm{Me}$ $R=H, R_{1}=R_{2}=M e$ $R_{1}=R_{2}=H, R=M e$ $R_{1}=H, R=R_{2}=M e$ $\mathrm{R}_{2}=\mathrm{H}, \mathrm{R}=\mathrm{R}_{1}=\mathrm{Me}$<smiles>[X]CCCc1ccc(P)o1</smiles>

$20 \mathrm{R}=\mathrm{H}, \mathrm{X}=\mathrm{Cl}$ $21 \mathrm{R}=\mathrm{Me}, X=\mathrm{Cl}$ $22 \mathrm{R}=\mathrm{H}, \mathrm{X}=\mathrm{I}$ $23 \mathrm{R}=\mathrm{Me}, \mathrm{X}=1$ d<smiles>[R2]C=C([R7])C(O)CCCc1ccc([R])o1</smiles>

24 25 26 27 28 29 30

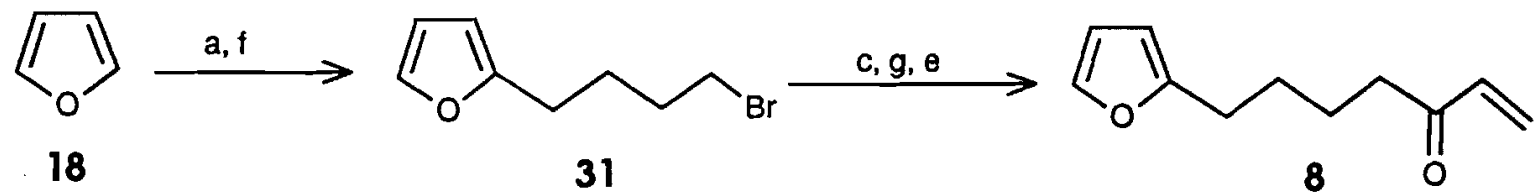

Reagents: (a) 1.2 equiv. $n-B u L i, T H F, 0^{\circ} \mathrm{C}, 1.5 \mathrm{~h}$; (b) 1-bromo-3-chloropropane; (c) Nal, acetone, reflux; (d) 2.2 equiv. $t-B u L i, \mathrm{Et}_{2} \mathrm{O},-78^{\circ} \mathrm{C}$; then, acrolein, methacrolein, crotonaldehyde or tiglic aldehyde; (e) Swern [O]; (f) 1,4-dibromobutane; (g) 2.2 equiv. $\mathrm{t}-\mathrm{BuLi}, \mathrm{Et}_{2} \mathrm{O},-78^{\circ} \mathrm{C}$; then acrolein

SCHEME 1

standard conditions (17) followed by trapping of the resultant anion with 1-bromo-3-chloropropane, afforded alkylated furans $20(63 \%)$ and $\mathbf{2 1}(95 \%)$, respectively. Conversion of the chlorides $\mathbf{2 0}$ and $\mathbf{2 1}$ into the iodides $\mathbf{2 2}$ and $\mathbf{2 3}$ was accomplished in excellent yield under Finkelstein conditions (18). Halogen-metal exchange of the iodides 22 and 23 with tert-butyllithium (19) followed by quenching of the resulting carbanion with acrolein, methacrolein, crotonaldehyde, or tiglic aldehyde provided allylic alcohols 24-30. Swern oxidation (20) of the allylic alcohols 24-30 yielded the IMDAF precursors 1-7 respectively. Furan 8 was prepared by reaction of the anion of furan $\mathbf{1 8}$ with 1,4-dibromobutane to provide bromide 31, which was converted to furan $\mathbf{8}$ by the identical sequence described above (Scheme 1).

The synthesis of IMDAF precursors 9-15 is summarized in Scheme 2. Ismail and Hoffman (21) have reported that Friedel-Crafts alkylation of either furan 18 or 2-methylfuran 19 with 4-methyl-2-oxo-3-pentenenitrile 32 (22) in the presence of aluminum trichloride in benzene provided esters 33 and $\mathbf{3 4}$, respectively, in reasonable yield after the reaction mixture was quenched with methanol. Our attempts to repeat this reaction provided esters $\mathbf{3 3}$ and $\mathbf{3 4}$ in poor yield $(<20 \%)$ due to the competing Friedel-Crafts alkylation of the solvent benzene. Changing the solvent to carbon disulfide provided esters 33 and 34 in 30 and $66 \%$ yield, respectively. The yield of ester $\mathbf{3 3}$ was diminished due the formation of the 2,5-dialkylated product 35. Attempts to prevent formation of 35 by blocking one $\alpha$-site of furan with a tert- butyldimethylsilyl moiety were unsuccessful; the silylated furan did not undergo Friedel-Crafts reactions when subjected to a variety of Friedel-Crafts conditions.

Although Lipshutz and co-workers (23a) reported successful 1,4 additions of the higher-order cyanocuprate of 2 -lithiofuran to $\alpha, \beta$-unsaturated ketones, we were unsuccessful in effecting the same reaction on 3-methyl-2-butenal, methyl 3-methyl-2-butenoate, or dimethyl isopropylidenemalonate. The failure of these reactions may be due to both the low reactivity of the cuprate reagent $(23 b)$, and to the hindered nature of the position to be attacked.

Reduction of esters $\mathbf{3 3}$ and $\mathbf{3 4}$ with lithium aluminum hydride provided alcohols 36 and 37 , respectively, which were converted to the corresponding iodides $\mathbf{3 8}$ and $\mathbf{3 9}$ via the tosylates (18). The iodides $\mathbf{3 8}$ and $\mathbf{3 9}$ were then converted to precursors 9-12 and 13-15 via: $(a)$ halogen-metal exchange, $(b)$ quenching the anion with an $\alpha, \beta$-unsaturated aldehyde to give allylic alcohols $\mathbf{4 0 - 4 6}$, and (c) Swern oxidation.

The acetylenic precursors were prepared by treating the anion formed by halogen-metal exchange of iodide 38 with either 3-(trimethylsilyl)propynal 47 (24) or 2-butynal 48 (Scheme 2) (25). Swern oxidation provided compounds $\mathbf{1 6}$ and $\mathbf{1 7}$ in good yield.

\section{Results and discussion}

Compound 2 was treated with a number of Lewis acids (26) that had been reported to accelerate both inter- $(9 c, 27)$ 
<smiles>CC(C)=CC(=O)O</smiles><smiles>CC(C)=CC(=O)C#N</smiles>

32

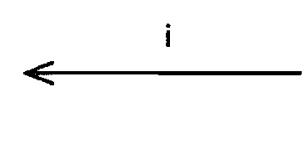

$\mathrm{R}=\mathrm{R}_{1}=\mathrm{R}_{2}=\mathrm{H}$

$$
\mathrm{R}=\mathrm{R}_{2}=\mathrm{H}, \mathrm{R}_{1}=\mathrm{Me}
$$

$\mathrm{R}=\mathrm{R}_{1}=\mathrm{H}, \mathrm{R}_{2}=\mathrm{Me}$

$\mathrm{R}=\mathrm{H}, \mathrm{R}_{1}=\mathrm{R}_{2}=\mathrm{Me}$

$\mathrm{R}_{1}=\mathrm{R}_{2}=\mathrm{H}, \mathrm{R}=\mathrm{Me}$

$\mathrm{R}_{2}=\mathrm{H}, \mathrm{R}=\mathrm{R}_{1}=\mathrm{Me}$

$\mathrm{R}_{1}=\mathrm{H}, \mathrm{R}=\mathrm{R}_{2}=\mathrm{Me}$<smiles>[X]CC(C)(C)c1ccc([R])o1</smiles>

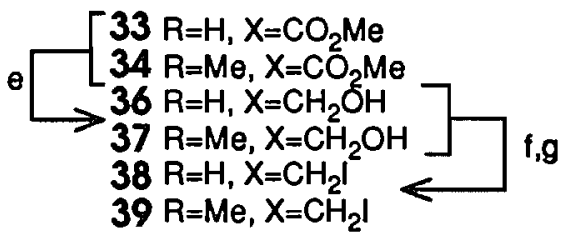<smiles>[R2]C=C([R7])C(O)CCC(C)(C)c1ccc([R])o1</smiles>

40

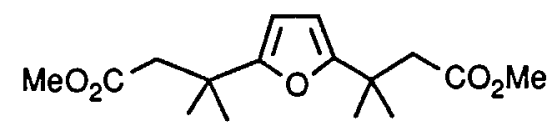

35
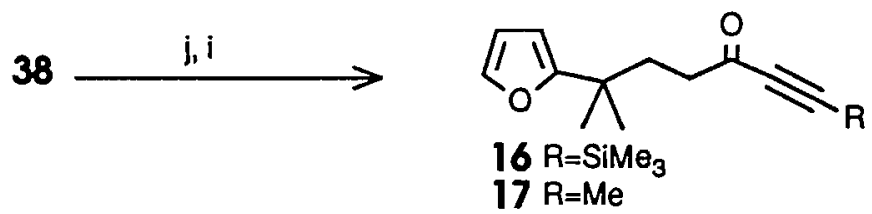

Reagents: (a) oxalyl choride, hexane; (b) $\mathrm{CuCN}, \mathrm{MeCN}$; (c) 0.25 equiv. $\mathrm{AlCl}_{3}, \mathrm{CS}_{2}$, furan 18 or 2-methylfuran 19; (d) $\mathrm{MeOH}$; (e) $\mathrm{LiAlH}_{4}, \mathrm{Et}_{2} \mathrm{O}$; (f) $\mathrm{TsCl}, \mathrm{DMAP}, \mathrm{CH}_{2} \mathrm{Cl}_{2}$; (g) Nal, acetone, reflux; (h) 2.2 equiv. $\mathrm{t}-\mathrm{BuLi}, \mathrm{Et}_{2} \mathrm{O}$, $-78^{\circ} \mathrm{C}$; then acrolein, m..thacrolein, crotonaldehyde, or tiglic aldehyde; (i) Swern [O]; (j) 2.2 equiv.t-BuLi, $\mathrm{Et}_{2} \mathrm{O}$, $-78^{\circ} \mathrm{C}$; then $\mathrm{TMSC} \equiv \mathrm{CCHO}(47)$ or $\mathrm{MeC} \equiv \mathrm{CCHO}(48)$.

SCHEME 2

and intra-molecular $(10 \mathrm{~m}, 13,28)$ Diels-Alder reactions (Table 1). Compound 2 was chosen for this model study since it had previously shown reluctance to undergo the IMDAF reaction when treated with Florisil either in methylene chloride or aqueous $2.0 \mathrm{M} \mathrm{CaCl}_{2}$ solutions (entries 9 and 10) (4h). In each example, 1.1 equivalents of Lewis acid was added to a dilute solution $(0.02-0.03 \mathrm{M})$ of compound 2 in methylene chloride at $-78^{\circ} \mathrm{C}$. Aliquots were removed from the reaction mixture and their ${ }^{1} \mathrm{H}$ NMR spectra were measured. The reaction was stirred at a particular temperature until there was no further change in the starting material to adduct (SM:A) ratio by ${ }^{1} \mathrm{H}$ NMR. The reaction was then warmed another 10 or $15^{\circ} \mathrm{C}$, and the process repeated until a temperature was reached at which decomposition was detected. The aliquots removed from the reaction mixture were quenched with cold $10 \%$ sodium bicarbonate to prevent reequilibration and to neutralize acidic by-products. The SM:A ratios were measured by integration of the ${ }^{1} H$ NMR spectrum of the crude reaction mixture, in particular by comparison of the integral of the vinyl protons of the adduct $\mathbf{5 0}(\delta$ 6.48 and 6.14$)$ and that due to the $\beta$-furan protons of the starting material $2(\delta 6.28$ and 6.00$)$.

The results are summarized in Table 1. Zinc iodide (entry
1) was the least effective Lewis acid examined, which was surprising in light of its proven synthetic utility for accelerating both the intermolecular Diels-Alder reaction of the furan diene $(9 c)$ and an IMDAF reaction in which the side arm contained three carbon atoms (13). Although some adduct was produced within $3 \mathrm{~h}$ at room temperature, the equilibrium still favoured starting material. The ratio of SM:A did not change significantly after $39 \mathrm{~h}$ and decomposition products were detected by 'H NMR. These findings are attributed to the unfavourable heterogeneous nature of the reaction mixture when zinc iodide is used. Tin(IV) chloride (entry 2) provided a favourable SM:A ratio of $24: 76$ within $1 \mathrm{~h}$ at $-78^{\circ} \mathrm{C}$; however, extensive decomposition was also observed (by 'H NMR). The remaining Lewis acids (entries 3-8) provided SM: A ratios favouring adduct after only $2 \mathrm{~h}$ at either -78 or $-50^{\circ} \mathrm{C}$; decomposition was evident at temperatures above $-50^{\circ} \mathrm{C}$ for all Lewis acids examined. The yields of the adduct were in excess of $95 \%$ (based on recovered starting material) after flash chromatography (29), indicating that decomposition below $-50^{\circ} \mathrm{C}$ was insignificant. For ease of handling and reaction work-up, methylaluminum dichloride was chosen as the Lewis acid to continue the study. 
TABLE 1. Effect of various Lewis acids on the IMDAF reaction of compound 2

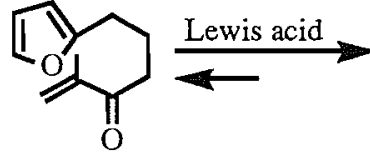

2

\begin{tabular}{|c|c|c|c|}
\hline & Lewis acid & Conditions & 2:50 Ratio \\
\hline 1 & $\mathrm{ZnI}_{2}$ & $\begin{array}{l}1 \mathrm{~h},-78^{\circ} \mathrm{C} \\
3 \mathrm{~h}, \text { r.t. } \\
39 \text { h, r.t. }\end{array}$ & $\begin{array}{c}100: 0 \\
69: 31 \\
65: 35^{a}\end{array}$ \\
\hline 2 & $\mathrm{SnCl}_{4}$ & $1 \mathrm{~h},-78^{\circ} \mathrm{C}$ & $24: 76^{b}$ \\
\hline 3 & $\mathrm{BF}_{3} \cdot \mathrm{Et}_{2} \mathrm{O}$ & $\begin{array}{l}2.5 \mathrm{~h},-78^{\circ} \mathrm{C} \\
3.5 \mathrm{~h},-50^{\circ} \mathrm{C}\end{array}$ & $\begin{array}{l}28: 72 \\
28: 72^{c}\end{array}$ \\
\hline 4 & $\mathrm{TiCl}_{4}: \mathrm{Ti}\left(\mathrm{O}^{\mathrm{i} P r}\right)_{4}$ & $\begin{array}{l}4.5 \mathrm{~h},-78^{\circ} \mathrm{C} \\
2.5 \mathrm{~h},-50^{\circ} \mathrm{C}\end{array}$ & $\begin{array}{l}32.68 \\
32.68^{d}\end{array}$ \\
\hline 5 & $\mathrm{EtAlCl}{ }_{2}$ & $\begin{array}{l}2.5 \mathrm{~h},-78^{\circ} \mathrm{C} \\
2.5 \mathrm{~h},-50^{\circ} \mathrm{C}\end{array}$ & $\begin{array}{l}35: 65 \\
35: 65^{d}\end{array}$ \\
\hline 6 & $\mathrm{MeAlCl}_{2}$ & $\begin{array}{l}2.5 \mathrm{~h},-78^{\circ} \mathrm{C} \\
2.5 \mathrm{~h},-50^{\circ} \mathrm{C}\end{array}$ & $\begin{array}{l}35: 65 \\
35: 65^{d}\end{array}$ \\
\hline 7 & $\mathrm{Et}_{2} \mathrm{AlCl}$ & $2.5 \mathrm{~h},-50^{\circ} \mathrm{C}$ & $35: 65$ \\
\hline 8 & $\mathrm{Me}_{2} \mathrm{AlCl}$ & $2.5 \mathrm{~h},-50^{\circ} \mathrm{C}$ & $32: 68$ \\
\hline 9 & Florisil $/ \mathrm{CH}_{2} \mathrm{Cl}_{2}$ & 14 days, r.t. or $40^{\circ} \mathrm{C}$ & $100: 0$ \\
\hline 10 & $2.0 \mathrm{M} \mathrm{CaCl}_{2}$ & 4 days, r.t. & $50: 50$ \\
\hline
\end{tabular}

"Some decomposition after $64 \mathrm{~h}$ (by 'H NMR).

"Extensive decomposition (by 'H NMR).

'Some decomposition after $3.5 \mathrm{~h}$ (by 'H NMR).

“Decomposition occurred above $-50^{\circ} \mathrm{C}$.

The effect of solvent on the Lewis acid-mediated IMDAF reaction was also examined. Treatment of compound $\mathbf{2}$ in hexane with methylaluminum dichloride resulted in the formation of a gummy precipitate. Both ${ }^{\mathrm{H}} \mathrm{H}$ NMR and TLC indicated that no IMDAF reaction had occurred. Toluene was also unsuitable as a solvent because many unidentified products formed even at $-78^{\circ} \mathrm{C}$. All subsequent reactions were therefore performed in methylene chloride.

The results from treating precursors 1-7 and 9-15 with methylaluminum dichloride at $-78^{\circ} \mathrm{C}$ are summarized in Tables 2 and 3 respectively. Previous results from our lab (4h) using Florisil are also included for comparison purposes. Several observations are noteworthy. The use of 1.1 equivalents of methylaluminum dichloride with precursors $\mathbf{1}, \mathbf{2}$, $\mathbf{5}$, and $\mathbf{6}$ provided their corresponding oxatricyclo adducts in excellent yields. Both compounds $\mathbf{1}$ and $\mathbf{5}$, which have unsubstituted dienophiles, provided adducts $\mathbf{4 9}$ and $\mathbf{5 3}$ in $99 \%$ yield after only $1 \mathrm{~h}$ at $-78^{\circ} \mathrm{C}$. Compounds 2 and $\mathbf{6}$, which were unreactive with Florisil, gave adducts $\mathbf{5 0}$ and $\mathbf{5 4}$ in 63 and $80 \%$ yields, respectively. In contrast, precursors $\mathbf{3}, \mathbf{4}$, and 7, which have substitution at the terminus of the dienophile, had SM: A ratios in favour of starting material. The unreactive nature of these dienophiles in the IMDAF reaction is well precedented in the literature (4i), and may be a result of both increased steric interactions in the transition state as well as increased electron density of the carboncarbon double bond.
The geminally substituted precursors $\mathbf{9}$ and $\mathbf{1 3}$ (Table 3) provided adducts 56 and $\mathbf{6 0}$ in $88 \%$ yield, respectively, upon treatment with Florisil alone after only 1 day at room temperature, and therefore were not treated with methylaluminum dichloride. Compounds 10, 11, 14, and 15 provided varying SM:A ratios when Florisil was employed; however, in all cases adduct was detected and isolated. This finding is in contrast to the results from the corresponding unsubstituted precursors $2,3,6$, and 7 (Table 1) in which no adduct was detected after 6 days. The increased reactivity of the substituted precursors may be due to the gem-dialkyl effect $(3 g, 3 h, 4 m, 8,30)$.

Treatment of compounds $10,11,14$, and 15 with 1.1 equivalents of methylaluminum dichloride provided SM:A ratios in favour of starting material; increased quantities of adduct were formed relative to the results from using Florisil, except for compound 14. The lower SM:A ratios for compounds 10 and 14 (Table 3) when compared to the SM:A ratios for compounds 2 and $\mathbf{6}$ (Table 2) can be explained by unfavourable 1,3-diaxial methyl interactions present in adducts 57 and 61, which are absent in adducts $\mathbf{5 0}$ and $\mathbf{5 4}$. Thus the 1,3-diaxial interactions may be promoting the retro DielsAlder reaction. Compound $\mathbf{1 2}$ provided no adduct with 1.1 equivalents of methylaluminum dichloride.

We then sought a means of overcoming the unfavourable equilibrium for precursors containing more hindered dienophiles. A survey of the literature revealed very few examples in which catalytic quantities of Lewis acids had been employed to accelerate IMDA reactions $(10 h, 10 j, 10 k, 28 c$, 31 ) and no examples involving the IMDAF reaction. Dramatic improvements in the SM:A ratios were observed when compounds 3, 6, 7 (Table 2), 10, 11, and 14 (Table 3) were exposed to 0.1 equivalents of methylaluminum dichloride at $-78^{\circ} \mathrm{C}$. In all cases the SM:A ratios favoured the adducts (SM:A ratios ranged from $40: 60$ to $0: 100$ ) and provided the oxatricyclo adducts in good to excellent yield. The SM:A ratios of compound $\mathbf{4}$ and $\mathbf{1 5}$ improved only slightly when compared to the results from the use of 1.1 equivalents of Lewis acid, and the tiglic precursor $\mathbf{1 2}$ did not react at all. These findings therefore represent the first examples of Lewis acid-catalyzed IMDAF reactions. In addition, these are also the first examples of Lewis acid-catalyzed IMDA reactions involving systems containing an internally activated dienophile.

The greatly improved SM:A ratios obtained when 0.1 equivalents of methylaluminum dichloride was employed, when compared with 1.1 equivalents, were rationalized by considering the effect that the amount of Lewis acid present has on the reaction equilibria. Our IMDAF reactions were shown to be under thermodynamic control: the reaction of pure adduct $\mathbf{5 0}$ with 1.1 equivalents of methylaluminum dichloride at $-78^{\circ} \mathrm{C}$ for $2.5 \mathrm{~h}$ provided the same ratio of compounds $2: 50(35: 65)$ that was obtained when pure precursor 2 was treated under the identical conditions (Scheme 3). The proposed equilibria are illustrated in Scheme 4. In the presence of 1.1 equivalents of Lewis acid the starting material and adduct are essentially complexed with Lewis acid and therefore both the forward and reverse Diels-Alder reactions are accelerated, leading to a thermodynamic ratio of Lewis acid complexed starting material $\mathbf{A}^{*}$ and adduct $\mathbf{B}^{*}$. Treatment of the reaction mixture with a bicarbonate quench at $-78^{\circ} \mathrm{C}$ destroys the Lewis acid and provides, in the case of compound 3, a SM:A ratio of 78:22 (Table 2). This ratio 
TABLE 2. Effect of methylaluminum dichloride on IMDAF reactions

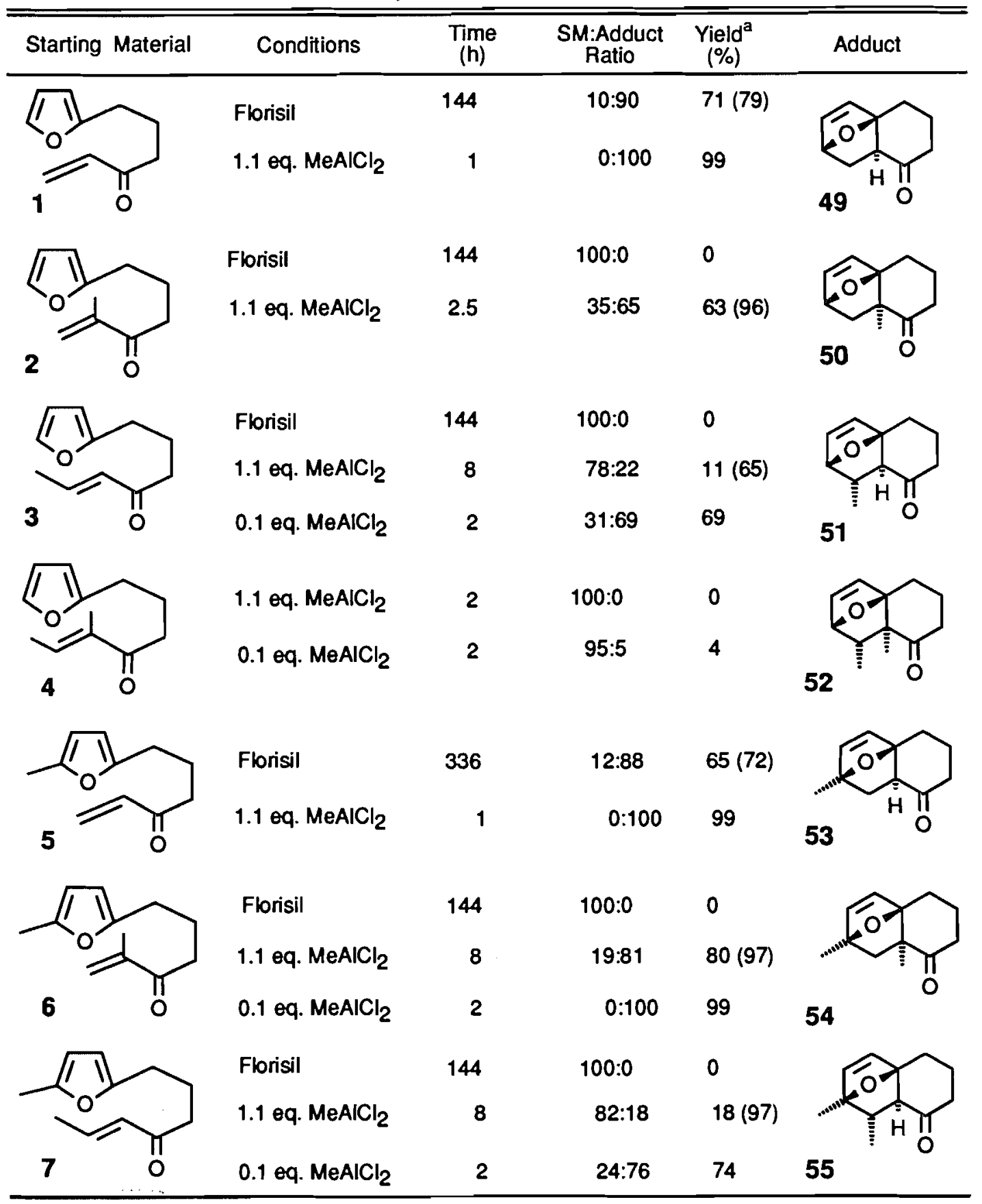

${ }^{a}$ Yield in parentheses is based on recovered starting material.

is therefore indicative of the $\mathbf{A}^{*}: \mathbf{B}^{*}$ ratio in solution, since it has been shown that compound $\mathbf{3}$ and adduct $\mathbf{5 1}$ do not interconvert in the absence of Lewis acid or upon work-up at room temperature. When catalytic quantities of methylaluminum dichloride are employed, the Lewis acid should preferentially complex with the more basic enone in the starting material A (Scheme 4) rather than with the saturated ketone in the adduct $\mathbf{B}$ (32). The dissociation of the Lewis acid from the adduct $\mathbf{B}^{*}$ and complexation with $\mathbf{A}$ thus not only slows the rate of the reverse Diels-Alder reaction but accelerates the forward reaction, leading to a shift in the equilibrium towards adduct. The SM: A ratio for compound 3:51 increased to $31: 69$ (Table 2). with 0.1 equivalents of
Lewis acid. Attempts to probe the nature of the species in solution by ${ }^{1} \mathrm{H}$ NMR studies have been inconclusive to date. Further work is in progress to establish support for the above hypothesis.

The utility of mild Lewis acids in the DMDAF reaction was not limited to either a four carbon atom side arm or dienophiles comprising enones. Compound 8, containing a five carbon atom side arm, provided two oxatricyclo adducts 63 and 64 in a ratio of 84:8 in favour of the endo isomer in addition to unreacted starting material when treated with 0.1 equivalents of methylaluminum dichloride at $-78^{\circ} \mathrm{C}$ for $2 \mathrm{~h}$ (Table 4). The stereochemistry of the major isomer $(63)$ was determined by comparison of the ${ }^{1} \mathrm{H}$ NMR spectrum of 63 
TABLE 3. Effect of $\mathrm{MeAlCl}_{2}$ on the IMDAF reaction of geminally substituted precursors

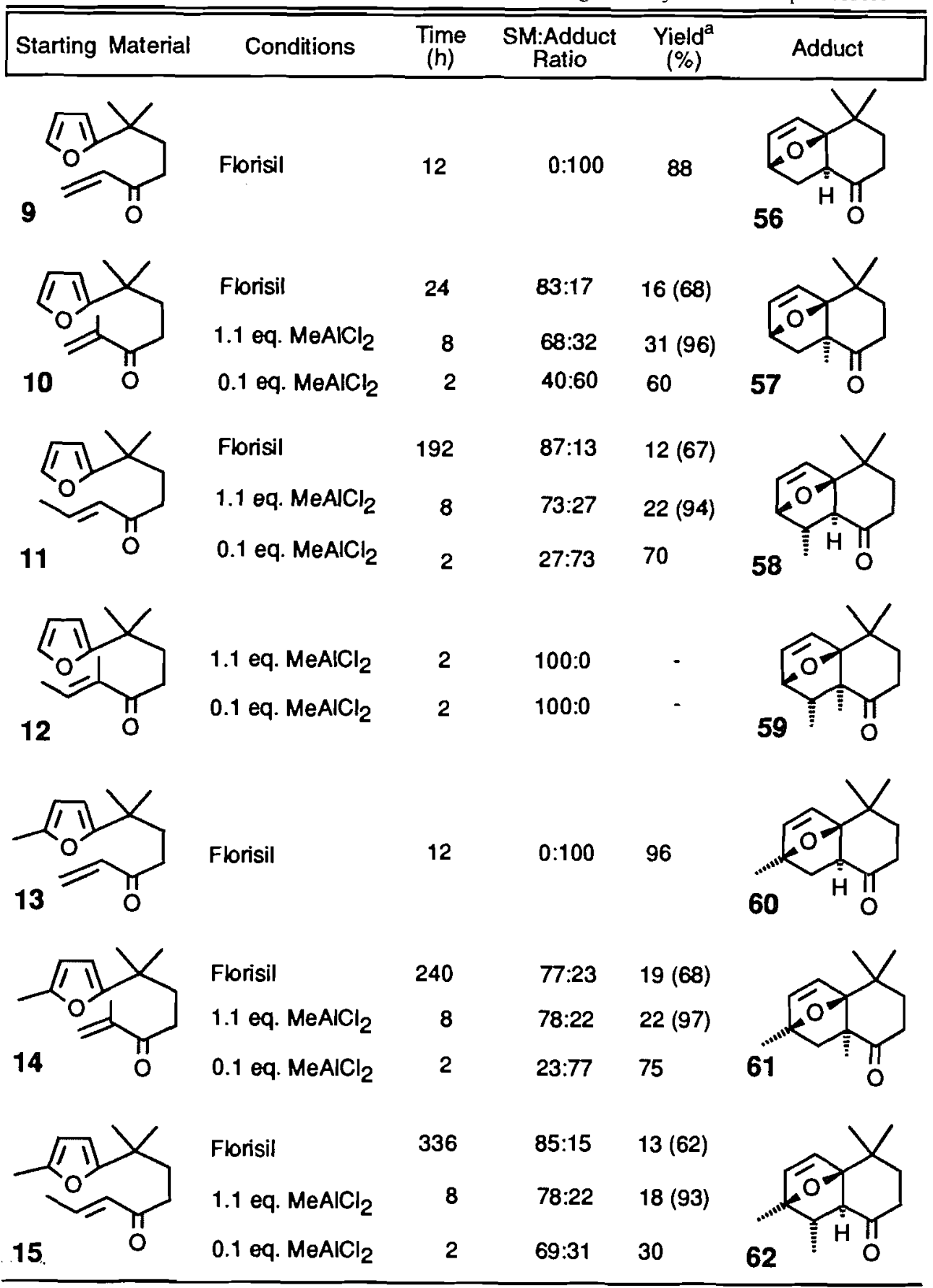

"Yield in parentheses is based on recovered starting material.

with that reported by Harwood and co-workers $(5 a)$. Interestingly, compound 8 has been previously reported to provide adducts 63 and 64 only at $1.2 \mathrm{GPa}$ and in a ratio of $1: 1$ $(5 a)$.

Acetylenic precursors 16 and 17 provided the strained adducts $65(88 \%)$ and $66(97 \%)$, respectively, when treated with 1.1 equivalents of dimethylaluminum chloride at $-50^{\circ} \mathrm{C}$ for $2.5 \mathrm{~h}$ (Scheme 5). The weaker Lewis acid dimethylaluminum chloride (26) was employed to minimize possible side reactions such as aromatization of the adducts. The use of internally activated acetylenic dienophiles in either the IMDA or IMDAF reaction is rare $(1,33)$, and no successful examples have been reported with the IMDAF reaction $(4 l)$. Usually the acetylene has been activated from the terminus by an ester moiety $(1,10)$. Thus, the reactions of compounds 16 and 17 are the first successful IMDA reactions involving internally activated acetylenic dienophiles. Adduct $\mathbf{6 5}$ was easily purified by flash chromatography, while adduct 66 was not as stable and partially isomerized to compound 67, which contains an exocyclic double bond, upon standing.

Stereochemistry of the adducts

The stereochemistry of all the adducts formed in the Lewis acid accelerated IMDAF reactions containing a four-carbon 
<smiles></smiles>

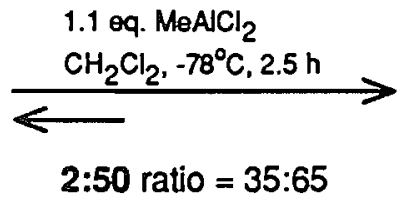

2

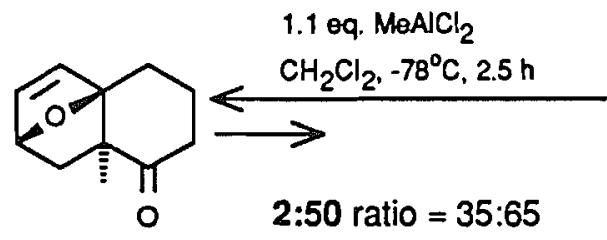

50

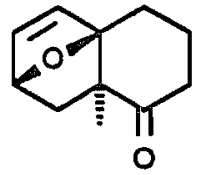

50

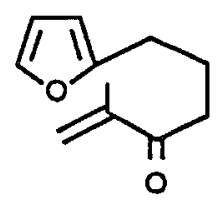

2
SCHEME 3

side arm had the side arm orientated syn with respect to the oxygen bridge resulting from an exo mode of attack. This is not surprising since (1) the calculated $\Delta G^{0}=+12.9 \mathrm{~kJ} / \mathrm{mol}$ (PCModel 4), for the equilibration between the exo adduct 49 and the corresponding endo adduct, favours the exo adduct and (2) the reactions are under thermodynamic control (vide supra). This stereochemistry is consistent with that reported by others in the literature for tethers consisting of four carbon atoms with an internally activated dienophile $(4 a, f)$. The delineation of the stereochemistry in adducts $\mathbf{4 9 - 5 1}$ and 53-55 (Scheme 6) will be described in detail as representative examples. The stereochemistry of the remaining adducts arising from either acrolein-, methacrolein-, or crotonaldehyde-type dienophiles were determined in a similar manner.

The stereochemistry of adducts 49 and $\mathbf{5 3}$ was determined by ${ }^{1} \mathrm{H}$ NMR based on the coupling constants of protons $\mathrm{H}-6 \alpha, \mathrm{H}-7 \alpha$, and $\mathrm{H}-7 \beta$ (Scheme 6 ). The bridge proton in adduct $49 \mathrm{H}-8(\delta 4.91)$ was coupled to both $\mathrm{H}-7 \beta$ ( $3.8 \mathrm{~Hz}$ ) and $\mathrm{H}-9(0.9 \mathrm{~Hz})$. Coupling of $\mathrm{H}-8$ to $\mathrm{H}-7 \alpha$ was not observed since the dihedral angle of $\mathrm{H}-8-\mathrm{C}-8-\mathrm{C}-7-\mathrm{H}-7 \alpha$ was $90^{\circ}$. Proton $\mathrm{H}-7 \beta(\delta 2.49)$ was a multiplet with couplings of $3.0 \mathrm{~Hz}, 3.8 \mathrm{~Hz}$, and $11.8 \mathrm{~Hz}$ to protons $\mathrm{H}-6 \alpha$ $(\delta 2.28), \mathrm{H}-8$, and $\mathrm{H}-7 \alpha(\delta 1.49)$, respectively. The $3.0 \mathrm{~Hz}$ coupling constant observed between $\mathrm{H}-7 \beta$ and $\mathrm{H}-6 \alpha$ was indicative of a $60^{\circ}$ dihedral angle, thereby placing the side arm syn to the oxygen bridge. If the side arm was orientated anti to the oxygen bridge then the $\mathrm{C}-6$ proton would have a dihedral angle of $0^{\circ} \mathrm{C}$ to the $\mathrm{C}-7 \alpha$ proton and a coupling constant of approximately $8 \mathrm{~Hz}$ would be expected (34). The $8.3 \mathrm{~Hz}$ coupling observed between $\mathrm{H}-7 \alpha$ and $\mathrm{H}-6 \alpha$ therefore confirmed our assignment of the relative stereochemistry. Since compound $\mathbf{5 3}$ has a methyl group at $\mathrm{C}-8$, proton $\mathrm{H}-7 \alpha$ was identified by its upfield shift as the doublet of doublets at $\delta 1.61$. This upfield shift was observed in compound 49 for $\mathrm{H}-7 \alpha$, and was probably due to the diamagnetic anisotropy of the carbon-carbon double bond. The coupling constants of $11.8 \mathrm{~Hz}$ to $\mathrm{H}-7 \beta$ and $8.2 \mathrm{~Hz}$ to $\mathrm{H}-6 \alpha$ again confirmed the $s y n$ orientation of the side arm to the oxygen bridge in compound $\mathbf{5 3}$.

The determination of the stereochemistry of the side arm with respect to the oxygen bridge in adducts $\mathbf{5 0}$ and $\mathbf{5 4}$ could not be directly related to coupling constants since a methyl group is attached at C-6 (Scheme 6). If the adducts were formed from the mode of cyclization in which the side arm was orientated exo, the C-6 methyl substituent would be anti (i.e., endo) to the oxygen bridge and be shielded by the C-9 C-10 double bond. The methyl group at C-6 had an upfield chemical shift of $\delta 1.11$ and $\delta 1.08$ for adducts $\mathbf{5 0}$ and 54, respectively, which is indicative of endo orientated methyl groups in oxatricyclo adducts (35). The proton $\mathrm{H}-7 \beta$ was a doublet of doublets at $\delta 2.86$ in compound $\mathbf{5 0}$, due to vicinal coupling with the bridge proton $\mathrm{H}-8(5.1 \mathrm{~Hz})$ and geminal coupling to $\mathrm{H}-7 \alpha(11.8 \mathrm{~Hz})$. Proton $\mathrm{H}-7 \alpha$ coupled only geminally to $\mathrm{H}-7 \beta(11.8 \mathrm{~Hz})$, and was highly shielded at $\delta 1.00$ due to the anisotropy of the carbon-carbon double bond. Similarly, protons $\mathrm{H}-7 \alpha$ and $\mathrm{H}-7 \beta$ appeared as an $\mathrm{AB}$ quartet $(11.8 \mathrm{~Hz})$ at $\delta 1.12$ and $\delta 2.55$, respectively, in compound 54.

The crotonaldehyde-derived adducts $\mathbf{5 1}$ and $\mathbf{5 5}$ contain an additional stereocenter at $\mathrm{C}-7$. Since IMDAF reactions catalyzed by Lewis acids have a concerted mechanism (36), the

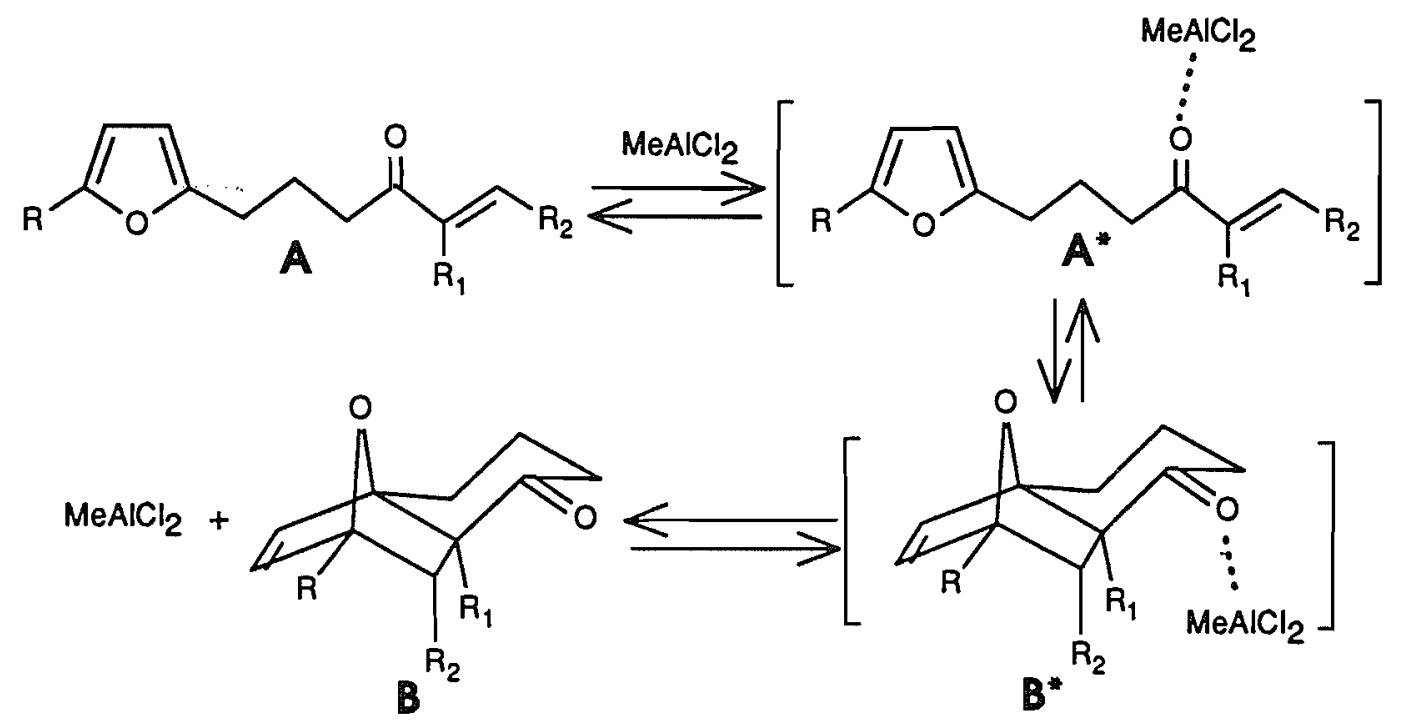

SCHEME 4 
TABLE 4. IMDAF reaction of compound 8

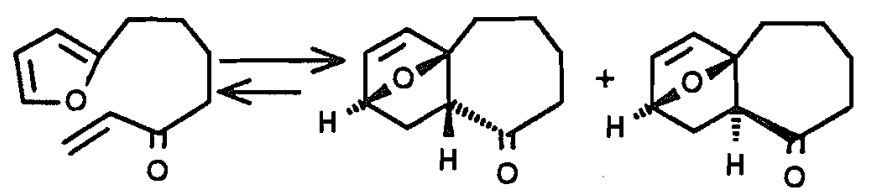

8

63 endo

64 exo

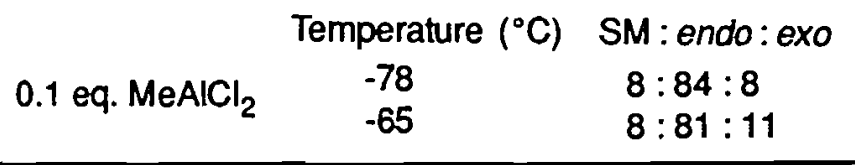

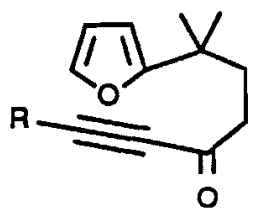

$\frac{1.1 \text { eq. } \mathrm{Me}_{2} \mathrm{AlCl}_{1}-50^{\circ} \mathrm{C}}{2.5 \mathrm{~h}, \mathrm{CH}_{2} \mathrm{Cl}_{2}}>$

\section{$16 \mathrm{R}=\mathrm{SiMe}_{3}$ $17 \mathrm{R}=\mathrm{Me}$}

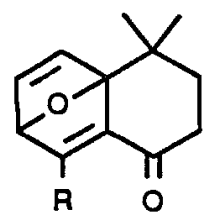

$65 \mathrm{R}=\mathrm{SiMe}_{3}(88 \%)$ $66 \mathrm{R}=\mathrm{Me}(>97 \%)$<smiles>C=C1C2C=CC3(O2)C1C(=O)CCC3(C)C</smiles>

67 methyl group at $\mathrm{C}-7$ should be anti to the oxygen bridge (Scheme 6) if an exo mode of cyclization had occurred. Proton H-8 ( $\delta 4.69)$ in adduct 51 was a doublet of doublets coupled to $\mathrm{H}-9(1.6 \mathrm{~Hz})$ and $\mathrm{H}-7 \beta(4.7 \mathrm{~Hz})$, indicating that the methyl group at C-7 was indeed anti to the oxygen bridge. If the C-7 methyl group was syn to the oxygen bridge, the protons $\mathrm{H}-8$ and $\mathrm{H}-7$ would form a $90^{\circ}$ dihedral angle, and would therefore show no coupling (34). This stereochemical assignment was also supported by the highly shielded chemical shift of the C-7 methyl doublet $(\delta 0.93)$ due to the anisotropy of the C-9-C-10 carbon-carbon double bond. Proton $\mathrm{H}-6$ was a doublet $(4.0 \mathrm{~Hz}, \delta 1.73)$ and coupled to $\mathrm{H}-7 \beta(\delta 2.79)$. The $4.0 \mathrm{~Hz}$ coupling constant was indicative of a $60^{\circ}$ dihedral angle, thereby placing H-6 anti, and the side arm syn, with respect to the oxygen bridge. The stereochemistry of compound $\mathbf{5 5}$ was assigned in a similar manner. The C-7 methyl group appeared as an upfield doublet at $\delta 0.95$, consistent with the moiety being anti to the oxygen bridge. Proton H-6 $(\delta 1.89)$ was a doublet with $4.2 \mathrm{~Hz}$ coupling to $\mathrm{H}-7$, indicating the side arm was syn with respect to the oxygen bridge.

\section{Conclusions}

We have shown that Lewis acids are effective reagents for promoting the IMDAF reaction in which the diene and dienophile are connected by four or five carbon atoms. The reaction conditions are also applicable to acetylenic dienophiles internally activated by a carbonyl moiety. The use of methylaluminum dichloride makes the IMDAF reaction a viable synthetic transformation since: (1) the time for the reaction to reach equilibrium is reduced to a few hours from 4 to 14 days; (2) the isolated yields of adducts are very high, and (3) only one adduct is formed with up to four asymmetric centres of known relative stereochemistry. Synthetic applications of this methodology are currently in progress.

\section{Experimental}

\section{General methods}

Melting points were determined using an Electrothermal melting point apparatus and are uncorrected. Boiling points refer to the air-bath temperature using Kugelrohr distillation apparatus, and are also uncorrected. Infrared spectra were obtained as either thin films (oils) on either $\mathrm{NaCl}$ or $\mathrm{KBr}$ plates, or as $\mathrm{KBr}$ pellets (solids). The infrared spectra were recorded on either a Nicolet 5-DX FT-IR spectrophotometer or a Mattson model 4030 FT-IR.

Nuclear magnetic resonance spectra were obtained on one of three instruments: Bruker AC-300 $\left({ }^{1} \mathrm{H} 300 \mathrm{MHz},{ }^{13} \mathrm{C} 75 \mathrm{MHz}\right)$, Bruker ACE-200 $\left({ }^{1} \mathrm{H} 200 \mathrm{MHz},{ }^{13} \mathrm{C} 50 \mathrm{MHz}\right)$, or a Bruker AM-400 spectrometer $\left({ }^{1} \mathrm{H} 400 \mathrm{MHz}\right)$. Deuterochloroform was used both as the solvent, and the internal standard $\left({ }^{1} \mathrm{H}, \delta 7.27 ;{ }^{13} \mathrm{C}, \delta 77.0\right)$ un-
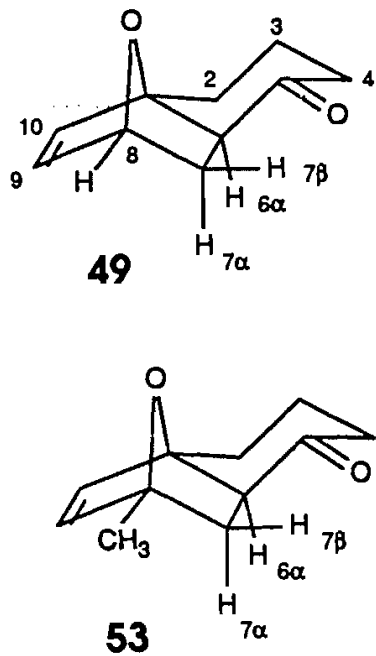

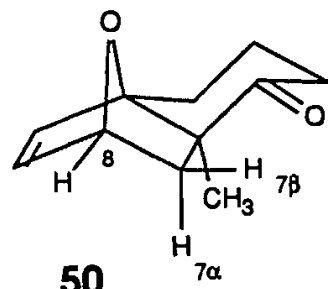

50

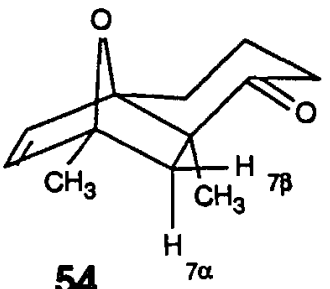

54

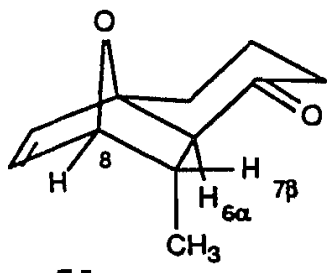

51

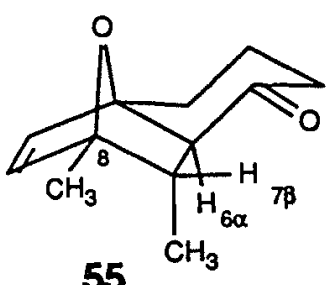

55

SCHEME 6 
less otherwise stated. All 'H NMR spectra listed will have the following format: chemical shift (in ppm), (multiplicity, number of protons, coupling constants $(\mathrm{Hz})$, assignment). The abbreviations used to describe the multiplicities are as follows: $b r=$ broadened, $\mathrm{s}=$ singlet, $\mathrm{d}=$ doublet, $\mathrm{t}=$ triplet, $\mathrm{q}=$ quartet, $\mathrm{qu}=$ quintet, and $A B q$ refers to a quartet due to an $A B$ spin system. The ${ }^{13} \mathrm{C} N M R$ spectra listed will have the following format: chemical shift (in $\mathrm{ppm}$ ), (number of attached protons as determined by DEPT experiments).

Low-resolution mass spectra were recorded using either a Varian $\mathrm{CH} 5$ spectrometer, or a VG 7070 instrument. The data are listed as mass $(m / e)$, (relative intensity, assignment). The spectra were obtained either by the electron-impact (E1) method or field ionization (FI), and the method will only be listed if FI was used. High-resolution mass spectra were recorded on a Kratos MS80 spectrometer. Microanalyses were performed either by Guelph Chemical Laboratories Limited, Guelph, Ontario, or by Mrs. D. Fox, Department of Chemistry, University of Calgary.

Solvents were either dried by standard methods (37), then distilled prior to use, or were purchased as anhydrous solvents in Sure-

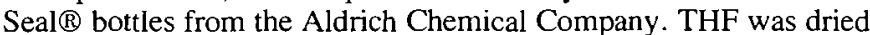
over sodium and benzophenone, as was diethyl ether. Methylene chloride, diisopropyl amine, and triethylamine were dried over $\mathrm{CaH}_{2}$. Methanol was refluxed with $\mathrm{Mg}^{0}$ metal for $12 \mathrm{~h}$, then distilled. DMSO was dried over $\mathrm{NaOH}$. Acetonitrile, DMF, and diethyl ether were purchased as anhydrous solvents, and HPLC-grade acetone was used.

The Lewis acids employed in this study were used as purchased, with no further purification. Boron trifluoride etherate and titanium(IV) chloride were used as neat liquids. The aluminumcontaining reagents were purchased as molar solutions in hexane from Aldrich.

All glassware and syringes were dried in a $120^{\circ} \mathrm{C}$ oven for at least $4 \mathrm{~h}$, then cooled under a stream of $\mathrm{Ar}$, or in a desiccator containing Drierite. Reactions that were sensitive to moisture or atmospheric conditions were performed under an Ar atmosphere.

General procedure 1: conversion of tosylate or ehloride to iodide

The tosylate or chloride $(10 \mathrm{mmol})$ and $\mathrm{Nal}(25 \mathrm{mmol})$ were dissolved in HPLC-grade acetone $(75 \mathrm{~mL})$ and heated to reflux for $24 \mathrm{~h}$ under Ar. The reaction was cooled, and the solvent removed in vacuo. Diethyl ether $(100 \mathrm{~mL})$ and water $(75 \mathrm{~mL})$ were then added to dissolve the crude material. The aqueous layer extracted with $\mathrm{Et}_{2} \mathrm{O}(3 \times 100 \mathrm{~mL})$ and the combined organic layers were dried over $\mathrm{Na}_{2} \mathrm{SO}_{4}$ (anhydrous), filtered, and the solvent removed in vacuo to provide the crude iodide. The iodide was purified either by flash chromatography, or distillation.

\section{General procedure 2: coupling of iodide with} unsaturated aldehydes

The iodide was purified by filtration through basic alumina, then distillation just prior to use. The iodide $(1.0 \mathrm{mmol})$ was dissolved in anhydrous $\mathrm{Et}_{2} \mathrm{O}(10 \mathrm{~mL})$, and the solution was cooled to $-78^{\circ} \mathrm{C}$ in a Dry Ice - acetone bath. tert-Butyllithium $(2.2 \mathrm{mmol})$ was added dropwise to the cooled solution by syringe, and the reaction was stirred for $1 \mathrm{~h}$ at $-78^{\circ} \mathrm{C}$. The $\alpha, \beta$-unsaturated aldehyde was distilled from $\mathrm{Na}_{2} \mathrm{SO}_{4}$ under $\mathrm{Ar}$, and was added neat $(1.5 \mathrm{mmol})$ by syringe to the reaction. The reaction was continued for a further 30 min (or until monitoring by TLC showed no starting material was present), then was quenched at low temperature with saturated $\mathrm{NH}_{4} \mathrm{Cl}(10 \mathrm{~mL})$. After warming to room temperature, the aqueous layer was extracted with $\mathrm{Et}_{2} \mathrm{O}(3 \times 25 \mathrm{~mL})$ and $\mathrm{CH}_{2} \mathrm{Cl}_{2}$ $(2 \times 25 \mathrm{~mL})$. The combined organic layers were dried over $\mathrm{Na}_{2} \mathrm{SO}_{4}$, filtered, and the solvent removed in vacuo to provide the crude coupled product, which was purified by flash chromatography, then distillation.

\section{General procedure 3: Swern oxidation}

The method of Swern (20) was used to oxidize the allylic alcohols to the enones. Oxalyl chloride $(11 \mathrm{mmol})$ was dissolved in dry $\mathrm{CH}_{2} \mathrm{Cl}_{2}(25 \mathrm{~mL})$ and cooled to $-60^{\circ} \mathrm{C}$ (Dry Ice - chloroform) in an Ar-purged 3-neck round-bottom flask equipped with a dropping funnel. Freshly distilled alcohol $(10 \mathrm{mmol})$ was dissolved in dry $\mathrm{CH}_{2} \mathrm{Cl}_{2}(10 \mathrm{~mL})$ and placed in the dropping funnel. DMSO ( $22 \mathrm{mmol}$ ) was added neat (by syringe) to the oxalyl chloride solution, the reaction was stirred for $2 \mathrm{~min}$, and then the alcohol solution was added within $5 \mathrm{~min}$. The mixture was stirred an additional $15 \mathrm{~min}$, then $\mathrm{Et}_{3} \mathrm{~N}$ ( $50 \mathrm{mmol}$ ) was added and stirring was continued for another $5 \mathrm{~min}$ before warming the reaction to room temperature. Water $(50 \mathrm{~mL})$ was added, and the aqueous layer was extracted with $\mathrm{CH}_{2} \mathrm{Cl}_{2}(5 \times 25 \mathrm{~mL})$. The combined organic layers were washed with $5 \% \mathrm{HCl}(15 \mathrm{~mL}), 5 \% \mathrm{Na}_{2} \mathrm{CO}_{3}(15 \mathrm{~mL})$, and water $(15 \mathrm{~mL})$, dried over $\mathrm{Na}_{2} \mathrm{SO}_{4}$, filtered, and the solvent removed in vacuo to provide the crude bad-smelling product, which was purified by flash chromatography, then distilled.

\section{General procedure 4: IMDAF reactions in Florisil}

The freshly distilled enone ( $1 \mathrm{mmol}$ ) was dissolved in dry $\mathrm{CH}_{2} \mathrm{Cl}_{2}$ $(15 \mathrm{~mL})$ and placed in an Ar-purged round-bottom flask. To this was added Florisil (100-200 mesh, 10 weight equivalents to the enone). The reaction was wrapped in foil and stirred at the appropriate temperature for the required length of time. The reaction was then filtered, the Florisil washed well with EtOAc $(20 \mathrm{~mL})$, and the solvent removed in vacuo without external heating to provide the crude product, which could be purified by flash chromatography.

\section{General procedure 5: IMDAF reactions using Lewis acid}

The freshly distilled enone $(0.2 \mathrm{mmol})$ was dissolved in dry $\mathrm{CH}_{2} \mathrm{Cl}_{2}(10 \mathrm{~mL})$, placed in an Ar-purged 3-necked flask, and cooled to the appropriate temperature. Methylaluminum dichloride $(1.0 \mathrm{M}$ in hexane) was added to the cooled enone solution, and the reaction was stirred for the appropriate length of time. The reaction was quenched with $10 \% \mathrm{NaHCO}_{3}(10 \mathrm{~mL})$, and warmed until no frozen material remained. The aqueous layer was extracted with $\mathrm{CH}_{2} \mathrm{Cl}_{2}(4 \times 10 \mathrm{~mL})$, then the organic layer was washed with $\mathrm{H}_{2} \mathrm{O}$ (15 mL), dried over $\mathrm{Na}_{2} \mathrm{SO}_{4}$, filtered, and the solvent removed in vacuo without external heating to provide the crude product, which was purified by flash chromatography.

\section{3-(2-Furyl)-1-chloropropane (20)}

Furan 18 (6.0 mL, $82.5 \mathrm{mmol})$ was dissolved in dry THF ( $140 \mathrm{~mL}$ ) under $\mathrm{Ar}$ and cooled to $-78^{\circ} \mathrm{C}$. Butyllithium $(2.5 \mathrm{M}$ in hexanes, $39.6 \mathrm{mmol}$ ) was added, then the solution was warmed to $0^{\circ} \mathrm{C}$ and stirred for $2 \mathrm{~h}$. The 1-bromo-3-chloropropane $(15.5 \mathrm{~g}$, $98.7 \mathrm{mmol}$ ) was passed through basic alumina, then distilled before being added dropwise to the yellow anion solution. The reaction was stirred at room temperature overnight. The solvent was removed in vacuo, then ether $(100 \mathrm{~mL})$ and saturated $\mathrm{NH}_{4} \mathrm{Cl}$ $(100 \mathrm{~mL})$ were added to the oily residue. The aqueous layer was extracted with ether $(3 \times 100 \mathrm{~mL})$, dried over $\mathrm{Na}_{2} \mathrm{SO}_{4}$, and the solvent removed in vacuo. Distillation under aspirator provided compound 20 as a clear, colourless oil $(9.1 \mathrm{~g}, 63.1 \mathrm{mmol}), 76 \%$ yield; bp $60-70^{\circ} \mathrm{C} / 20$ Torr $(1$ Torr $=133.3 \mathrm{~Pa})$; IR (neat) $\mathrm{cm}^{-1}$ : 2959-2850(C-H), 735(C-Cl); ' $\mathrm{H}$ NMR (200 MHz): 2.12(qu, 2H, $J=7.0 \mathrm{~Hz}), 2.82(\mathrm{t}, 2 \mathrm{H}, J=7.0 \mathrm{~Hz}), 3.57(\mathrm{t}, 2 \mathrm{H}, J=7.0 \mathrm{~Hz})$, $6.05(\mathrm{dd}, 1 \mathrm{H}, J=0.8 \mathrm{~Hz}, J=3.0 \mathrm{~Hz}), 6.30(\mathrm{dd}, 1 \mathrm{H}, J=1.8 \mathrm{~Hz}$, $J=3.0 \mathrm{~Hz}), 7.33(\mathrm{dd}, 1 \mathrm{H}, J=0.8 \mathrm{~Hz}, J=1.8 \mathrm{~Hz}) ;{ }^{13} \mathrm{C} \mathrm{NMR}$ $(50 \mathrm{MHz}): 25.07(\mathrm{t}), 30.86(\mathrm{t}), 44.01(\mathrm{t}), 105.55(\mathrm{~d}), 110.09(\mathrm{~d})$, $141.13(\mathrm{~d}), 154.29(\mathrm{~s})$; mass spectrum: $144\left(15, \mathrm{M}^{\circ+}\right), 81(100$, $\left.\mathrm{M}-\left(\mathrm{CH}_{2}\right)_{2} \mathrm{Cl}\right)$. Exact Mass calcd. for $\mathrm{C}_{7} \mathrm{H}_{9} \mathrm{ClO}$ : 144.0342 ; found: 144.0352

\section{3-(2-(5-Methylfuryl))-1-chloropropane (21)}

2-Methylfuran $19(7.9 \mathrm{~mL}, 80.0 \mathrm{mmol})$ was dissolved in dry THF (140 mL) under Ar and cooled to $-78^{\circ} \mathrm{C}$. Butyllithium $(2.5 \mathrm{M}$ in hexanes, $38.4 \mathrm{mmol}$ ) was added, then the solution was warmed to $0^{\circ} \mathrm{C}$ and stirred for $2 \mathrm{~h}$. The 1-bromo-3-chloropropane $(17.0 \mathrm{~g}$, $108.2 \mathrm{mmol}$ ) was passed through basic alumina, then distilled before being added dropwise to the yellow anion solution. The reaction was stirred at room temperature overnight. The solvent was removed in vacuo, then ether $(100 \mathrm{~mL})$ and saturated $\mathrm{NH}_{4} \mathrm{Cl}$ $(100 \mathrm{~mL})$ were added to the oily residue. The aqueous layer was 
extracted with ether $(3 \times 100 \mathrm{~mL})$, dried over $\mathrm{Na}_{2} \mathrm{SO}_{4}$, and the solvent removed in vacuo. Distillation under aspirator provided chloride 21 as a clear, colourless oil $(12.3 \mathrm{~g}, 77.5 \mathrm{mmol}), 97 \%$ yield, bp $75-85^{\circ} \mathrm{C} / 20$ Torr; IR (neat) $\mathrm{cm}^{-1}$ : 2956-2867(C-H), 653(C-Cl); 'H NMR (200 MHz): $2.10(\mathrm{qu}, 2 \mathrm{H}, J=6.9 \mathrm{~Hz}), 2.27(\mathrm{~s}$, $3 \mathrm{H}), 2.76(\mathrm{t}, 2 \mathrm{H}, J=7.2 \mathrm{~Hz}), 3.58(\mathrm{t}, 2 \mathrm{H}, J=6.5 \mathrm{~Hz}), 5.87(\mathrm{~d}$, $1 \mathrm{H}, J=3.0 \mathrm{~Hz}), 5.92(\mathrm{~d}, 1 \mathrm{H}, J=3.0 \mathrm{~Hz}) ;{ }^{13} \mathrm{C} \mathrm{NMR}(50 \mathrm{MHz})$ : 13.27(q), 25.02(t), 30.87(t), 43.96(t), 105.66(d), 106.02(d), 150.43(s), 152.25(s); mass spectrum: $158\left(11, \mathrm{M}^{++}\right), 95(100, \mathrm{M}-$ $\left.\left(\mathrm{CH}_{2}\right)_{2} \mathrm{Cl}\right)$. Exact Mass calcd. for $\mathrm{C}_{8} \mathrm{H}_{11} \mathrm{OCl}$ : 158.0498; found: 158.0501

\section{3-(2-Furyl)-1-iodopropane (22)}

Using general procedure 1 , chloride $20(9.1 \mathrm{~g}, 63.1 \mathrm{mmol})$ was converted to iodide $22(14.1 \mathrm{~g}, 59.7 \mathrm{mmol})$ in $95 \%$ yield after distillation; bp 20-30 $\mathrm{C} / 0.04$ Torr; IR (neat) $\mathrm{cm}^{-1}$ : 3144-3001(C-H), 2953-2843(C-H); ${ }^{1} \mathrm{H}$ NMR $(200 \mathrm{MHz}): 2.15(\mathrm{qu}, 2 \mathrm{H}, J=$ $7.0 \mathrm{~Hz}), 2.77(\mathrm{t}, 2 \mathrm{H}, J=7.0 \mathrm{~Hz}), 3.20(\mathrm{t}, 2 \mathrm{H}, J=7.0 \mathrm{~Hz})$, $6.06(\mathrm{dd}, 1 \mathrm{H}, J=0.8 \mathrm{~Hz}, J=3.1 \mathrm{~Hz}), 6.29(\mathrm{dd}, 1 \mathrm{H}, J=1.8 \mathrm{~Hz}$, $J=3.1 \mathrm{~Hz}), 7.32(\mathrm{dd}, 1 \mathrm{H}, J=0.8 \mathrm{~Hz}, J=1.8 \mathrm{~Hz}) ;{ }^{13} \mathrm{C} \mathrm{NMR}$ $(50 \mathrm{MHz}): 5.79(\mathrm{t}), 28.57(\mathrm{t}), 31.61(\mathrm{t}), 105.70(\mathrm{~d}), 110.09(\mathrm{~d})$, 141.16(d), 153.92(s); mass spectrum: 236(51, $\left.\mathrm{M}^{*+}\right), 81(100$, $\left.\mathrm{M}-\left(\mathrm{CH}_{2}\right)_{2} \mathrm{I}\right)$. Exact Mass calcd. for $\mathrm{C}_{7} \mathrm{H}_{9} \mathrm{IO}: 235.9697$; found: 235.9704 .

\section{3-(2-(5-Methylfuryl))-1-iodopropane (23)}

Using general procedure 1 , chloride $21(3.75 \mathrm{~g}, 23.6 \mathrm{mmol})$ was converted to the iodide $23(5.60 \mathrm{~g}, 22.4 \mathrm{mmol})$ in $95 \%$ yield after distillation; bp $100^{\circ} \mathrm{C} / 20$ Torr; IR (neat) $\mathrm{cm}^{-1}$ : 3102(C-H), 2942$2845(\mathrm{C}-\mathrm{H})$; 'H NMR $(300 \mathrm{MHz}) ; 2.13(q u, 2 \mathrm{H}, J=6.8 \mathrm{~Hz})$, $2.26(\mathrm{~s}, 3 \mathrm{H}), 2.71(\mathrm{t}, 2 \mathrm{H}, J=6.8 \mathrm{~Hz}), 3.21(\mathrm{t}, 2 \mathrm{H}, J=6.8 \mathrm{~Hz})$, 5.85 and $5.89(\mathrm{ABq}, 2 \mathrm{H}, J=3.0 \mathrm{~Hz}) ;{ }^{13} \mathrm{C}$ NMR $(50 \mathrm{MHz}): 5.88(\mathrm{t})$, $13.42(\mathrm{q}), 28.63(\mathrm{t}), 31.72(\mathrm{t}), 105.78(\mathrm{~d}), 106.28(\mathrm{~d}), 150.50(\mathrm{~s})$, 151,94(s): mass spectrum: $250\left(51, \mathrm{M}^{*+}\right), 95\left(100, \mathrm{M}-\left(\mathrm{CH}_{2}\right)_{2} \mathrm{I}\right)$. Exact Mass calcd. for $\mathrm{C}_{8} \mathrm{H}_{11} \mathrm{IO}$; 249.9855 ; found: 249.9857 .

\section{6-(2-Furyl)-1-hexen-3-ol (24)}

Using general procedure 2 , iodide $22(0.59 \mathrm{~g}, 2.50 \mathrm{mmol})$ was treated with tert-butyllithium $(3.23 \mathrm{~mL}, 5.5 \mathrm{mmol})$ and acrolein $(0.25 \mathrm{~mL}, 3.74 \mathrm{mmol})$ to produce compound $24(0.22 \mathrm{~g}$, $1.32 \mathrm{mmol}$ ) as a clear, colourless oil in $53 \%$ yield after purification by flash chromatography $(5: 1)$ and distillation; bp $60-68^{\circ} \mathrm{C} /$ 0.04 Torr; IR (neat) $\mathrm{cm}^{-1}: 3385(\mathrm{O}-\mathrm{H}) ;{ }^{1} \mathrm{H}$ NMR $(200 \mathrm{MHz}): 1.52-$ 1.84(overlapping $\mathrm{m}, 6 \mathrm{H}), 2.67(\mathrm{t}, 2 \mathrm{H}, J=7.1 \mathrm{~Hz}), 4.13(\mathrm{~m}, 1 \mathrm{H}$, $J=6.2 \mathrm{~Hz}$ ), $5.12(\mathrm{ddd}, 1 \mathrm{H}, J=1.3 \mathrm{~Hz}, J=1.3 \mathrm{~Hz}, J=$ $10.3 \mathrm{~Hz}$ ), 5.23 (ddd, $1 \mathrm{H}, J=1.3 \mathrm{~Hz}, J=1.3 \mathrm{~Hz}, J=17.2 \mathrm{~Hz}$ ), 5.90 (ddd, $1 \mathrm{H}, J=6.2 \mathrm{~Hz}, J=10.3 \mathrm{~Hz}, J=17.2 \mathrm{~Hz}$ ), $6.00(\mathrm{dd}$, $1 \mathrm{H}, J=0.8 \mathrm{~Hz}, J=3.1 \mathrm{~Hz}), 6.28(\mathrm{dd}, 1 \mathrm{H}, J=1.8 \mathrm{~Hz}, J=$ $3.1 \mathrm{~Hz}), 7.30(\mathrm{dd}, 1 \mathrm{H}, J=0.8 \mathrm{~Hz}, J=1.8 \mathrm{~Hz}) ;{ }^{13} \mathrm{C} \mathrm{NMR}$ $(50 \mathrm{MHz}): 23.87(\mathrm{t}), 27.80(\mathrm{t}), 36.39(\mathrm{t}), 72.93(\mathrm{~d}), 104.85(\mathrm{~d})$, $110.05(\mathrm{~d}), \quad 114.72(\mathrm{t}), 140.77(\mathrm{~d}), 141.09(\mathrm{~d}), 155.98(\mathrm{~s})$; mass spectrum: $166\left(9, \mathrm{M}^{*+}\right), \quad 148\left(10, \mathrm{M}-\mathrm{H}_{2} \mathrm{O}\right), 94(100, \mathrm{M}-$ $\left.\mathrm{CH}_{2}=\mathrm{C}(\mathrm{OH}) \mathrm{CH}=\mathrm{CH}_{2}\right)_{\text {. . Exact }}$ Mass calcd. for $\mathrm{C}_{10} \mathrm{H}_{14} \mathrm{O}_{2}$ : 166.0994; found: 166.0994 .

\section{6-(2-Furyl)-2-methyl-1-hexen-3-ol (25)}

Using general procedure 2 , iodide $22(0.51 \mathrm{~g}, 2.18 \mathrm{mmol})$ was treated with tert-butyllithium $(2.82 \mathrm{~mL}, 4.79 \mathrm{mmol})$ and methacrolein $(0.27 \mathrm{~mL}, 3.26 \mathrm{mmol})$ to produce compound $25(0.22 \mathrm{~g}$, $1.20 \mathrm{mmol}$ ) as a clear, colourless oil in $55 \%$ yield after purification by flash chromatography $(5: 1)$ and distillation; bp $130^{\circ} \mathrm{C} /$ 0.1 Torr; IR (neat) $\mathrm{cm}^{-1}: 3397(\mathrm{OH}){ }^{1} \mathrm{H}$ NMR $(200 \mathrm{MHz}): 1.55-$ $1.80(\mathrm{~m}, 5 \mathrm{H}), 1.72(\mathrm{t}, 3 \mathrm{H}, J=1.1 \mathrm{~Hz}, J=1.1 \mathrm{~Hz}), 2.67(\mathrm{t}, 2 \mathrm{H}$, $J=7.2 \mathrm{~Hz}, \mathrm{H}-4), 4.08(\mathrm{br} \mathrm{q}, 1 \mathrm{H}), 4.85(\mathrm{qu}, 1 \mathrm{H}, J=1.6 \mathrm{~Hz})$, $4.95(\mathrm{~m}, 1 \mathrm{H}, J=1.0 \mathrm{~Hz}, J=1.6 \mathrm{~Hz}), 5.99(\mathrm{dd}, 1 \mathrm{H}, J=0.8 \mathrm{~Hz}$, $J=3.1 \mathrm{~Hz}), 6.28(\mathrm{dd}, 1 \mathrm{H}, J=1.8 \mathrm{~Hz}, J=3.1 \mathrm{~Hz}), 7.30(\mathrm{dd}, 1 \mathrm{H}$, $J=0.8 \mathrm{~Hz}, J=1.8 \mathrm{~Hz}) ;{ }^{13} \mathrm{C}$ NMR $(50 \mathrm{MHz}): 17.45(\mathrm{q}), 24.06(\mathrm{t})$, $27.76(\mathrm{t}), 34.22(\mathrm{t}), 75.85(\mathrm{~d}), 104.82(\mathrm{~d}), 110.03(\mathrm{~d}), 111.10(\mathrm{t})$, $140.75(\mathrm{~d}), 147.40(\mathrm{~s}), 156.00(\mathrm{~s})$; mass spectrum: $180\left(10, \mathrm{M}^{++}\right)$, 94(100, $\mathrm{M}-\mathrm{CH}_{2} \mathrm{CH}(\mathrm{OH}) \mathrm{C}(\mathrm{Me})=\mathrm{CH}_{2}$, McLafferty rearr.). Exact Mass calcd. for $\mathrm{C}_{11} \mathrm{H}_{16} \mathrm{O}_{2}$ : 180.1151 ; found: 180.1150 .

\section{(E-7-(2-Furyl)-2-hepten-4-ol (26)}

Using general procedure 2, iodide $22(0.60 \mathrm{~g}, 2.56 \mathrm{mmol})$ was treated with tert-butyllithium $(3.32 \mathrm{~mL}, 5.64 \mathrm{mmol})$ and crotonaldehyde $(0.32 \mathrm{~mL}, 3.84 \mathrm{mmol})$ to produce compound $\mathbf{2 6}$ $(0.31 \mathrm{~g}, 1.72 \mathrm{mmol})$ as a clear, colourless oil in $67 \%$ yield after purification by flash chromatography $(9: 1)$ and distillation; bp 52$68^{\circ} \mathrm{C} / 0.035$ Torr; IR (neat) $\mathrm{cm}^{-1}: 3443(\mathrm{O}-\mathrm{H})$; ' $\mathrm{H}$ NMR $(200 \mathrm{MHz}): 1.47-1.84(\mathrm{~m}, 5 \mathrm{H}), 1.70(\mathrm{dd}, 3 \mathrm{H}, J=0.9 \mathrm{~Hz}, J=$ $6.0 \mathrm{~Hz}), 2.65(\mathrm{t}, 2 \mathrm{H}, J=7.1 \mathrm{~Hz}), 4.06(\mathrm{br} \mathrm{q}, 1 \mathrm{H}, J=6.3 \mathrm{~Hz})$, $5.48(\mathrm{ddq}, 1 \mathrm{H}, J=0.9 \mathrm{~Hz}, J=6.0 \mathrm{~Hz}, J=15.3 \mathrm{~Hz}), 5.67(\mathrm{ddq}$, $1 \mathrm{H}, J=1.5 \mathrm{~Hz}, J=6.8 \mathrm{~Hz}, J=15.3 \mathrm{~Hz}), 5.99(\mathrm{dd}, 1 \mathrm{H}, J=$ $0.8 \mathrm{~Hz}, J=3.1 \mathrm{~Hz}), 6.28(\mathrm{dd}, 1 \mathrm{H}, 1.9 \mathrm{~Hz}, J=3.1 \mathrm{~Hz}), 7.30(\mathrm{dd}$, $1 \mathrm{H}, J=0.8 \mathrm{~Hz}, J=1.9 \mathrm{~Hz}) ;{ }^{13} \mathrm{C} \operatorname{NMR}(50 \mathrm{MHz}): 17.62(\mathrm{q})$, $24.00(\mathrm{t}), 27.81(\mathrm{t}), 36.63(\mathrm{t}), 72.84(\mathrm{~d}), 104.78(\mathrm{~d}), 110.02(\mathrm{~d})$, 126.97(d), 134.11(d), 140.72(d), 156.05(s); mass spectrum: 180(8, $\left.\mathrm{M}^{*+}\right), \quad 162\left(39, \mathrm{M}-\mathrm{H}_{2} \mathrm{O}\right), \quad 94\left(100, \mathrm{M}-\mathrm{CH}_{2} \mathrm{CH}\left(\mathrm{OH}_{2}\right)-\right.$ $\mathrm{CH}=\mathrm{CHMe}$ ). Anal. calcd. for $\mathrm{C}_{11} \mathrm{H}_{16} \mathrm{O}_{2}: \mathrm{C} 73.30, \mathrm{H} 8.95$; found: C 73.10, H 9.02

\section{(E)-7-(2-Furyl)-3-methyl-2-hepten-4-ol (27)}

Using general procedure 2, iodide $22(1.56 \mathrm{~g}, 6.63 \mathrm{mmol})$ was treated with tert-butyllithium $(8.58 \mathrm{~mL}, 14.58 \mathrm{mmol})$ and tiglic aldehyde $(0.96 \mathrm{~mL}, 9.94 \mathrm{mmol})$ to produce compound 27 $(0.58 \mathrm{~g}, 3.00 \mathrm{mmol})$ as a clear, colourless oil in $45 \%$ yield after purification by flash chromatography $(7: 1)$ and distillation; bp 72$78^{\circ} \mathrm{C} / 0.1$ Torr; IR (neat) $\mathrm{cm}^{-1}: 3365(\mathrm{O}-\mathrm{H}) ;{ }^{1} \mathrm{H}$ NMR $(200 \mathrm{MHz})$ : $1.49(\mathrm{br}, \mathrm{s}, 1 \mathrm{H}), 1.50-1.80(\mathrm{~m}, 10 \mathrm{H}), 2.65(\mathrm{t}, 2 \mathrm{H}, J=5.0 \mathrm{~Hz})$, 4.01 (br m, 1H), 5.47(br q, $1 \mathrm{H}), 5.99(\mathrm{dd}, 1 \mathrm{H}, J=0.7 \mathrm{~Hz}, J=$ $3.1 \mathrm{~Hz}), 6.28(\mathrm{dd}, 1 \mathrm{H}, J=1.9 \mathrm{~Hz}, J=3.1 \mathrm{~Hz}), 7.30(\mathrm{dd}, 1 \mathrm{H}$, $J=0.7 \mathrm{~Hz}, J=1.9 \mathrm{~Hz})$; mass spectrum: $194\left(23, \mathrm{M}^{++}\right), 176(42$, $\left.\mathrm{M}-\mathrm{H}_{2} \mathrm{O}\right), 94\left(100, \mathrm{M}-\mathrm{CH}_{2} \mathrm{CH}(\mathrm{OH}) \mathrm{C}(\mathrm{Me})=-\mathrm{CHMe}\right)$. Anal. calcd. for $\mathrm{C}_{12} \mathrm{H}_{18} \mathrm{O}_{2}: \mathrm{C} 74.19, \mathrm{H} 9.34$; found: $\mathrm{C} 73.91, \mathrm{H} 9.32$.

\section{6-(2-(5-Methylfuryl))-I-hexen-3-ol (28)}

Using general procedure 2, iodide $23(0.59 \mathrm{~g}, 2.34 \mathrm{mmol})$ was treated with tert-butyllithium $(3.03 \mathrm{~mL}, 5.15 \mathrm{mmol})$ and acrolein $(0.24 \mathrm{~mL}, 3.51 \mathrm{mmol})$ to produce compound $28(0.25 \mathrm{~g}$, $1.40 \mathrm{mmol}$ ) as a clear, colourless oil in $60 \%$ yield after purification by flash chromatography $(5: 1)$ and distillation; bp $130-135^{\circ} \mathrm{C} /$ 12 Torr; IR (neat) $\mathrm{cm}^{-1}: 3400(\mathrm{C}-\mathrm{O}), 1021(\mathrm{C}-\mathrm{O})$; 'H NMR (200 MHz): 1.53-1.81(overlapping $\mathrm{m}, 6 \mathrm{H}), 2.26(\mathrm{~s}, 1 \mathrm{H}), 2.61(\mathrm{t}$, $2 \mathrm{H}, J=7.2 \mathrm{~Hz}), 4.13(\mathrm{br}, \mathrm{m}, 1 \mathrm{H}, J=6.2 \mathrm{~Hz}), 5.12(\mathrm{dt}, 1 \mathrm{H}, J=$ $1.3 \mathrm{~Hz}, J=1.3 \mathrm{~Hz}, J=10.3 \mathrm{~Hz}), 5.23(\mathrm{dt}, 1 \mathrm{H}, J=1.3 \mathrm{~Hz}$, $J=1.3 \mathrm{~Hz}, J=17.2 \mathrm{~Hz}), 5.85(\mathrm{ABq}, 2 \mathrm{H}), 5.87(\mathrm{ddd}, 1 \mathrm{H}, J=$ $6.2 \mathrm{~Hz}, J=10.3 \mathrm{~Hz}, J=17.2 \mathrm{~Hz}) ;{ }^{13} \mathrm{C}$ NMR $(50 \mathrm{MHz}): 13.41$ (q), $23.96(\mathrm{t}), 27.85(\mathrm{t}), 36.42(\mathrm{t}), 72.93(\mathrm{~d}), 105.40(\mathrm{~d}), 105.73(\mathrm{~d})$, $114.73(\mathrm{t}), 141.10(\mathrm{~d}), 150.16(\mathrm{~s}), 154.08(\mathrm{~s})$; mass spectrum: $180(31$, $\left.\mathrm{M}^{++}\right), 162\left(7, \mathrm{M}-\mathrm{H}_{2} \mathrm{O}\right), 108\left(100, \mathrm{M}-\mathrm{CH}_{2}=\mathrm{C}(\mathrm{OH}) \mathrm{CH}=\mathrm{CH}_{2}\right)$. Anal. calcd. for $\mathrm{C}_{11} \mathrm{H}_{16} \mathrm{O}_{2}: \mathrm{C} 73.30, \mathrm{H} \mathrm{8.95}$; found: $\mathrm{C} 73.08$, H 9.21 .

\section{6-(2-(5-Methylfuryl))-2-methyl-1-hexen-3-ol (29)}

Using general procedure 2, iodide $23(0.52 \mathrm{~g}, 2.07 \mathrm{mmol})$ was treated with tert-butyllithium $(2.67 \mathrm{~mL}, 4.55 \mathrm{mmol})$ and methacrolein $(0.26 \mathrm{~mL}, 3.10 \mathrm{mmol})$ to produce compound $29(0.28 \mathrm{~g}$, $1.45 \mathrm{mmol}$ ) as a clear, colourless oil in $70 \%$ yield after purification by flash chromatography $(5: 1)$ and distillation; bp $69-77^{\circ} \mathrm{C} /$ 0.04 Torr; IR (neat) $\mathrm{cm}^{-1}: 3405(\mathrm{O}-\mathrm{H})$; ' $\mathrm{H}$ NMR (200 MHz): 1.55$1.70(\mathrm{~m}, 5 \mathrm{H}), 1.72(\mathrm{dd}, 3 \mathrm{H}, J=0.9 \mathrm{~Hz}, J=1.2 \mathrm{~Hz}), 2.25(\mathrm{~s}, 3 \mathrm{H})$, $2.61(\mathrm{t}, 2 \mathrm{H}, 7.0 \mathrm{~Hz}), 4.08(\mathrm{br} \mathrm{t}, 1 \mathrm{H}, J=7.5 \mathrm{~Hz}), 4.84(\mathrm{~m}, 1 \mathrm{H}, J=$ $1.7 \mathrm{~Hz}), 4.95(\mathrm{~m}, 1 \mathrm{H}, J=0.8 \mathrm{~Hz}, J=1.7 \mathrm{~Hz}), 5.85(\mathrm{ABq}, 2 \mathrm{H})$ ${ }^{13} \mathrm{C}$ NMR (50 MHz): 13.42(q), 17.44(q), 24.19(t), 27.84(t), 34.35(t), 75.66(d), 105.40(d), 105.79(d), 110.99(t), 147.48(s), 150.13(s), 154.16(s); mass spectrum: $194\left(14, \mathrm{M}^{++}\right), 108(100, \mathrm{M}$ $\left.-\mathrm{CH}_{2} \mathrm{CH}(\mathrm{OH}) \mathrm{C}(\mathrm{Me})=\mathrm{CH}_{2}\right)$. Anal. calcd. for $\mathrm{C}_{12} \mathrm{H}_{18} \mathrm{O}_{2}: \mathrm{C} 74.19$, H 9.34; found: C 73.82, H 9.38.

\section{(E)-7-(2-(5-Methylfuryl))-2-hepten-4-ol (30)}

Using general procedure 2, iodide $23(0.79 \mathrm{~g}, 3.18 \mathrm{mmol})$ was treated with tert-butyllithium $(4.10 \mathrm{~mL}, 6.99 \mathrm{mmol})$ and crotonaldehyde $(0.53 \mathrm{~mL}, 6.35 \mathrm{mmol})$ to produce compound $\mathbf{3 0}$ 
$(0.52 \mathrm{~g}, 2.66 \mathrm{mmol})$ as a clear, colourless oil in $84 \%$ yield after purification by flash chromatography $(9: 1)$ and distillation; bp 72$78^{\circ} \mathrm{C} / 0.1$ Torr; IR (neat) $\mathrm{cm}^{-1}: 3417(\mathrm{O}-\mathrm{H}) ;{ }^{1} \mathrm{H}$ NMR $(200 \mathrm{MHz})$ : $1.50-1.70(\mathrm{~m}, 5 \mathrm{H}), 1.70(\mathrm{dd}, 3 \mathrm{H}, J=0.9 \mathrm{~Hz}, J=6.3 \mathrm{~Hz}), 2.25(\mathrm{~s}$, $3 \mathrm{H}), 2.58(\mathrm{t}, 2 \mathrm{H}, J=7.5 \mathrm{~Hz}), 4.06(\mathrm{br} \mathrm{m}, 1 \mathrm{H}), 5.46(\mathrm{ddq}, 1 \mathrm{H}, J=$ $1.2 \mathrm{~Hz}, J=6.8 \mathrm{~Hz}, J=15.3 \mathrm{~Hz}), 5.68(\mathrm{ddq}, 1 \mathrm{H}, J=0.9 \mathrm{~Hz}$, $J=5.9 \mathrm{~Hz}, J=15.3 \mathrm{~Hz}), 5.85(\mathrm{ABq}, 2 \mathrm{H}) ;{ }^{13} \mathrm{C}$ NMR $(50 \mathrm{MHz})$ : 13.46(q), $\quad 17.82(\mathrm{q}), \quad 24.12(\mathrm{t}), \quad 27.88(\mathrm{t}), \quad 36.68(\mathrm{t}), \quad 72.88(\mathrm{~d})$, 105.35(d), 105.72(d), 126.91(d), 134.14(d), 150.12(s), 154.20(s); mass spectrum: $194\left(16, \mathrm{M}^{*+}\right), 178\left(36, \mathrm{M}-\mathrm{H}_{2} \mathrm{O}\right), 108(100, \mathrm{M}-$ $\mathrm{CH}_{2} \mathrm{CH}(\mathrm{OH}) \mathrm{CH}=\mathrm{CHMe}$ ). Anal. calcd. for $\mathrm{C}_{12} \mathrm{H}_{18} \mathrm{O}_{2}: \mathrm{C} 74.19, \mathrm{H}$ 9.34; found: C 73.86, H 9.29.

\section{6-(2-Furyl)-1-hexen-3-one (1)}

Compound $24(0.19 \mathrm{~g}, 1.18 \mathrm{mmol})$ was oxidized according to general procedure 3 to provide compound $1(0.18 \mathrm{~g}, 1.10 \mathrm{mmol})$ as a clear, colourless oil in $93 \%$ yield after purification by flash chromatography $(20: 1)$ and distillation; bp $50-55^{\circ} \mathrm{C} / 0.08$ Torr. The ${ }^{1} \mathrm{H}$ NMR spectrum always contained $>10 \%$ bridged adduct 49 , therefore compound 1 was not analyzed but treated with either Florisil or methylaluminum dichloride to provide adduct 49.

\section{6-(2-Furyl)-2-methyl-1-hexen-3-one (2)}

Compound $25(0.65 \mathrm{~g}, 3.58 \mathrm{mmol})$ was oxidized according to general procedure 3 to provide compound $2(0.60 \mathrm{~g}, 3.36 \mathrm{mmol})$ as a clear, colourless oil in $94 \%$ yield after purification by flash chromatography $(20: 1)$ and distillation; bp $58-60^{\circ} \mathrm{C} / 0.045 \mathrm{Torr}$; $\mathrm{JR}$ (neat) $\mathrm{cm}^{-1}: 1677(\mathrm{C}=\mathrm{O})$; ${ }^{1} \mathrm{H}$ NMR $(200 \mathrm{MHz}): 1.87(\mathrm{dd}$, $3 \mathrm{H}, J=0.8 \mathrm{~Hz}, J=1.4 \mathrm{~Hz}), 1.97(\mathrm{qu}, 2 \mathrm{H}, J=7.3 \mathrm{~Hz}), 2.67(\mathrm{t}$, $2 \mathrm{H}, J=7.3 \mathrm{~Hz}), 2.72(\mathrm{t}, 2 \mathrm{H}, J=7.3 \mathrm{~Hz}), 5.75(\mathrm{dq}, 1 \mathrm{H}, J=$ $0.8 \mathrm{~Hz}, J=1.4 \mathrm{~Hz}), 5.92(\mathrm{~m}, 1 \mathrm{H}, J=1.4 \mathrm{~Hz}), 6.00(\mathrm{dd}, 1 \mathrm{H}, J=$ $0.8 \mathrm{~Hz}, J=3.0 \mathrm{~Hz}), 6.28(\mathrm{dd}, 1 \mathrm{H}, J=2.0 \mathrm{~Hz}, J=3.0 \mathrm{~Hz})$, $7.30(\mathrm{dd}, 1 \mathrm{H}, J=0.8 \mathrm{~Hz}, J=2.0 \mathrm{~Hz}) ;{ }^{13} \mathrm{C} \operatorname{NMR}(50 \mathrm{MHz})$ : $17.55(\mathrm{q}), \quad 22.80(\mathrm{t}), 27.27(\mathrm{t}), 36.44(\mathrm{t}), \quad 105.18(\mathrm{~d}), \quad 110.06(\mathrm{~d})$, $124.27(\mathrm{t}), \quad 140.91(\mathrm{~d}), 144.53(\mathrm{~s}), 155.46(\mathrm{~s}), 199.52(\mathrm{~s})$; mass spectrum: $178\left(21, \mathrm{M}^{+}\right), 94\left(100, \mathrm{M}-\mathrm{CH}_{2}=\mathrm{C}(\mathrm{OH}) \mathrm{C}(\mathrm{Me})=\mathrm{CH}_{2}\right.$, McLafferty rearr.). Exact Mass calcd. for $\mathrm{C}_{11} \mathrm{H}_{14} \mathrm{O}_{2}$ : 178.0994; found: 178.0998 .

\section{(E)-7-(2-Furyl)-2-hepten-4-one (3)}

Compound $26(0.27 \mathrm{~g}, 1.48 \mathrm{mmol})$ was oxidized according to general procedure 3 to provide compound $3(0.23 \mathrm{~g}, 1.29 \mathrm{mmol})$ as a clear, colourless oil in $88 \%$ yield after purification by flash chromatography (20:1) and distillation; bp $60-70^{\circ} \mathrm{C} / 0.05$ Torr; IR (neat) $\mathrm{cm}^{-1}: 1672$ and $1696(\mathrm{C}=0)$; ' $\mathrm{H}$ NMR $(200 \mathrm{MHz}): 1.89(\mathrm{dd}$, $3 \mathrm{H}, J=1.6 \mathrm{~Hz}, J=6.8 \mathrm{~Hz}), 1.96(\mathrm{qu}, 2 \mathrm{H}, J=7.2 \mathrm{~Hz}), 2.57(\mathrm{t}$, $2 \mathrm{H}, J=7.2 \mathrm{~Hz}), 2.66(\mathrm{t}, 2 \mathrm{H}, J=7.2 \mathrm{~Hz}), 6.00(\mathrm{dd}, 1 \mathrm{H}, J=$ $0.6 \mathrm{~Hz}, J=3.1 \mathrm{~Hz}), 6.11(\mathrm{dq}, 1 \mathrm{H}, J=1.6 \mathrm{~Hz}, J=15.7 \mathrm{~Hz})$, $6.28(\mathrm{dd}, 1 \mathrm{H}, J=1.9 \mathrm{~Hz}, J=3.0 \mathrm{~Hz}), 6.83(\mathrm{dq}, 1 \mathrm{H}, J=6.8 \mathrm{~Hz}$, $J=15.7 \mathrm{~Hz}), 7.30(\mathrm{dd}, 1 \mathrm{H}, J=0.6 \mathrm{~Hz}, J=1.9 \mathrm{~Hz}) ;{ }^{13} \mathrm{C} \mathrm{NMR}$ $(50 \mathrm{MHz}): 18.09(\mathrm{q}), 22.50(\mathrm{t}), 27.26(\mathrm{t}), 38.92(\mathrm{t}), 105.18(\mathrm{~d})$, 110.04 (d), 131.9l(d), 140.89(d), 142.31(d), 155.44(s), 199.74(s); mass spectrum (FI): $178\left(100, \mathrm{M}^{*+}\right)$. Anal. calcd. for $\mathrm{C}_{11} \mathrm{H}_{14} \mathrm{O}_{2}: \mathrm{C}$ 74.13, H 7.92; found: C 74.18, H 7.98.

\section{(E)-7-(2-Furyl)-3-methyl-2-hepten-4-one (4)}

Compound $27(0.69 \mathrm{~g}, 3.55 \mathrm{mmol})$ was oxidized according to general procedure 3 to provide compound $4(0.62 \mathrm{~g}, 3.24 \mathrm{mmol})$ as a clear, colourless oil in $91 \%$ yield after purification by flash chromatography $(20: 1)$ and distillation; bp $60-70^{\circ} \mathrm{C} / 0.045 \mathrm{Torr}$; IR (neat) $\mathrm{cm}^{-1}: 1666(\mathrm{C}=\mathrm{O})$; ' $\mathrm{H}$ NMR $(200 \mathrm{MHz}): 1.77(\mathrm{~d}, 3 \mathrm{H}$, $J=1.1 \mathrm{~Hz}), 1.84(\mathrm{~d}, 3 \mathrm{H}, J=6.9 \mathrm{~Hz}), 1.96(\mathrm{qu}, 2 \mathrm{H}, J=7.2 \mathrm{~Hz})$, 2.66 and 2.68 (overlapping t, $4 \mathrm{H}, J=7.2 \mathrm{~Hz}$ ), 5.99(dd, $1 \mathrm{H}, J=$ $0.7 \mathrm{~Hz}, J=3.1 \mathrm{~Hz}), 6.27(\mathrm{dd}, 1 \mathrm{H}, J=1.8 \mathrm{~Hz}, J=3.1 \mathrm{~Hz})$, $6.69(\mathrm{qq}, 1 \mathrm{H}, J=1.1 \mathrm{~Hz}, J=6.9 \mathrm{~Hz}), 7.29(\mathrm{dd}, 1 \mathrm{H}, J=0.7 \mathrm{~Hz}$, $J=1.8 \mathrm{~Hz}) ;{ }^{13} \mathrm{C}$ NMR $(50 \mathrm{MHz}): 10.78(\mathrm{q}), 14.51(\mathrm{q}), 22.92(\mathrm{t})$, 27.19(t), 35.90(t), 104.94(d), 109.90(d), 136.88(d), 138.02(s), 140.68(d), 155.40(s), 200.94(s); mass spectrum (FI): 192(51, $\left.\mathrm{M}^{+}\right)$, $94(100-\mathrm{CH}=\mathrm{C}(\mathrm{OH}) \mathrm{C}(\mathrm{Me})=\mathrm{CHMe}$, McLafferty rearrangement). Anal. calcd. for $\mathrm{C}_{12} \mathrm{H}_{16} \mathrm{O}_{2}: \mathrm{C} 74.97, \mathrm{H}$ 8.39; found: C $74.85, \mathrm{H} 8.43$.
6-(2-(5-Methylfuryl)-1-hexen-3-one (5)

Compound $28(0.24 \mathrm{~g}, 1.34 \mathrm{mmol})$ was oxidized according to general procedure 3 to provide compound $5(0.15 \mathrm{~g}, 0.84 \mathrm{mmol})$ as a clear, colourless oil in $63 \%$ yield after purification by flash chromatography $(20 ; 1)$ and distillation; bp $60-70^{\circ} \mathrm{C} / 0.04$ Torr; IR (neat) $\mathrm{cm}^{-1}: 1684(\mathrm{C}=\mathrm{O}) ;{ }^{1} \mathrm{H}$ NMR $(200 \mathrm{MHz}): 1.96(\mathrm{qu}, 2 \mathrm{H}, J=$ $7.5 \mathrm{~Hz}), 2.25(\mathrm{~s}, 3 \mathrm{H}), 2.59-2.70(\mathrm{t}, 2 \mathrm{H}$ each, $J=7.5 \mathrm{~Hz}), 5.85(\mathrm{~m}$, $2 \mathrm{H}), 6.19(\mathrm{dd}, 1 \mathrm{H}, J=1.5 \mathrm{~Hz}, J=16.5 \mathrm{~Hz}), 6.38(\mathrm{dd}, 1 \mathrm{H}, J=$ $10.1 \mathrm{~Hz}, J=16.5 \mathrm{~Hz}) ;{ }^{13} \mathrm{C}$ NMR $(50 \mathrm{MHz}): 13.42(\mathrm{q}), 22.33(\mathrm{t})$, $27.24(\mathrm{t}), 38.57(\mathrm{t}), 105.74(\mathrm{~d}), 105.86(\mathrm{~d}), 127.87(\mathrm{t}), 136.50(\mathrm{~d})$, 150.36(s), 153.36(s), 200.34(s); mass spectrum: 178(13, $\left.\mathrm{M}^{+}\right)$, $108\left(100, \mathrm{M}-\mathrm{CH}_{2}=\mathrm{C}(\mathrm{OH}) \mathrm{CH}=\mathrm{CH}_{2}\right.$, McLafferty rearrangement)

\section{6-(2-(5-Methylfuryl))-2-methyl-1-hexen-3-one (6)}

Compound $29(0.28 \mathrm{~g}, 1.45 \mathrm{mmol})$ was oxidized according to general procedure 3 to provide compound $6(0.27 \mathrm{~g}, 1.42 \mathrm{mmol})$ as a clear, colourless oil in $98 \%$ yield after purification by flash chromatography $(20: 1)$ and distillation; bp $60-68^{\circ} \mathrm{C} / 0.045 \mathrm{Torr}$; IR (neat) $\mathrm{cm}^{-1}: 1680(\mathrm{C}=\mathrm{O}) ;{ }^{\mathrm{H}} \mathrm{H}$ NMR $(200 \mathrm{MHz}): 1.87(\mathrm{dd}, 3 \mathrm{H}$, $J=0.7 \mathrm{~Hz}, J=1.3 \mathrm{~Hz}), 1.94(\mathrm{qu}, 2 \mathrm{H}, J=7.3 \mathrm{~Hz}), 2.24(\mathrm{~s}, 3 \mathrm{H})$, $2.61(\mathrm{t}, 2 \mathrm{H}, J=7.3 \mathrm{~Hz}), 2.73(\mathrm{t}, 2 \mathrm{H}, J=7.3 \mathrm{~Hz}), 5.75(\mathrm{~m}, 1 \mathrm{H}$, $J=0.7 \mathrm{~Hz}, J=1.4 \mathrm{~Hz}), 5.84(\mathrm{ABq}, 2 \mathrm{H}), 5.93(\mathrm{~m}, 1 \mathrm{H}, J=$ $1.3 \mathrm{~Hz}, J=1.4 \mathrm{~Hz}) ;{ }^{13} \mathrm{C}$ NMR $(50 \mathrm{MHz}): 13.41(\mathrm{q}), 17.54(\mathrm{q})$, $22.95(\mathrm{t}), 27.37(\mathrm{t}), 36.52(\mathrm{t}), 105.78(\mathrm{~d}), 124.19(\mathrm{t}), 144.57(\mathrm{~s})$, $150.33(\mathrm{~s}), 153.56(\mathrm{~s}), 201.61(\mathrm{~s})$; mass spectrum: 192(62, $\left.\mathrm{M}^{++}\right)$, $108\left(100, \mathrm{M}-\mathrm{CH}_{2}=\mathrm{C}(\mathrm{OH}) \mathrm{C}(\mathrm{Me})=\mathrm{CH}_{2}\right.$, McLafferty rearrangement). Exact Mass calcd. for $\mathrm{C}_{12} \mathrm{H}_{16} \mathrm{O}_{2}: 192.1150$; found: 192.1158 .

\section{(E)-7-(2-(5-Methylfuryl))-2-hepten-4-one (7)}

Compound $30(0.30 \mathrm{~g}, 1.54 \mathrm{mmol})$ was oxidized according to general procedure 3 to provide compound $7(0.24 \mathrm{~g}, 1.23 \mathrm{mmol})$ as a clear, colourless oil in $80 \%$ yield after purification by flash chromatography $(20: 1)$ and distillation; bp $70-82^{\circ} \mathrm{C} / 0.045$ Torr; IR (neat) $\mathrm{cm}^{-1}: 1695-1670(\mathrm{C}=\mathrm{O}) ;{ }^{1} \mathrm{H}$ NMR $(200 \mathrm{MHz}): 1.89(\mathrm{dd}$, $3 \mathrm{H}, J=2.5 \mathrm{~Hz}, J=7.5 \mathrm{~Hz}), 1.95(\mathrm{qu}, 2 \mathrm{H}, J=7.0 \mathrm{~Hz}), 2.25(\mathrm{~s}$, $3 \mathrm{H}), 2.55(\mathrm{t}, 2 \mathrm{H}, J=7.0 \mathrm{~Hz}), 2.60(\mathrm{t}, 2 \mathrm{H}, J=7.0 \mathrm{~Hz}), 5.84(\mathrm{ABq}$, $2 \mathrm{H}), 6.11(\mathrm{dq}, 1 \mathrm{H}, J=1.6 \mathrm{~Hz}, J=15.8 \mathrm{~Hz}), 6.83(\mathrm{dq}, 1 \mathrm{H}, J=$ $6.8 \mathrm{~Hz}, J=15.8 \mathrm{~Hz}) ;{ }^{13} \mathrm{C}$ NMR $(50 \mathrm{MHz}): 13.39(\mathrm{q}), 18.08(\mathrm{q})$, $22.59(\mathrm{t}), 27.38(\mathrm{t}), 38.96(\mathrm{t}), 105.74(\mathrm{~d}), 105.78(\mathrm{~d}), 131.90(\mathrm{~d})$, 142.24(d), 150.28(s), 153.49(s), 199.88(s); mass spectrum: 192(10, $\left.\mathrm{M}^{++}\right), 108\left(100, \mathrm{M}-\mathrm{CH}_{2}=\mathrm{C}(\mathrm{OH}) \mathrm{CH}=\mathrm{CHMe}\right.$, McLafferty rearrangement). Exact Mass calcd. for $\mathrm{C}_{12} \mathrm{H}_{16} \mathrm{O}_{2}$ : 192.1151; found: 192.1138.

\section{1-Bromo-4-(2-furyl)butane (31)}

Furan $18(5.0 \mathrm{~mL}, 68.7 \mathrm{mmol})$ was dissolved in dry THF $(80 \mathrm{~mL})$ in an Ar-purged flask and cooled to $-78^{\circ} \mathrm{C}$ in a Dry Ice acetone bath. To this solution was added $n$-butyllithium $(20.0 \mathrm{~mL}, 50.0 \mathrm{mmol})$, then the reaction was stirred at room temperature for $1 \mathrm{~h}$. Freshly distilled 1,4-dibromobutane $(10.0 \mathrm{~g}$, $46.3 \mathrm{mmol}$ ) was added to the orange anion, and the reaction was stirred overnight. Saturated $\mathrm{NH}_{4} \mathrm{Cl}(50 \mathrm{~mL})$ was used to quench the reaction. The aqueous layer was extracted with ether $(3 \times$ $100 \mathrm{~mL}$ ), then the combined organic layers were dried over $\mathrm{Na}_{2} \mathrm{SO}_{4}$, filtered, and the solvent removed in vacuo. The crude product was distilled using a fractionating column under aspirator pressure, collecting the fraction boiling from 95 to $100^{\circ} \mathrm{C}$ to give compound $31(2.54 \mathrm{~g}, 12.5 \mathrm{mmol})$ in $25 \%$ yield; IR (neat) $\mathrm{cm}^{-1}: 2943-$ 2864(C-H); 'H NMR (200 MHz): 1.67-2.08(m, 4H), 2.68(t, $2 \mathrm{H}$, $J=7.1 \mathrm{~Hz}), 3.43(\mathrm{t}, 2 \mathrm{H}, J=7.1 \mathrm{~Hz}), 6.02(\mathrm{dd}, 1 \mathrm{H}, J=0.8 \mathrm{~Hz}$, $J=3.0 \mathrm{~Hz}), 6.30(\mathrm{dd}, 1 \mathrm{H}, J=1.7 \mathrm{~Hz}, J=3.0 \mathrm{~Hz}), 7.33(\mathrm{dd}, 1 \mathrm{H}$, $J=0.8 \mathrm{~Hz}, J=1.7 \mathrm{~Hz}) ;{ }^{13} \mathrm{C}$ NMR $(50 \mathrm{MHz}): 26.56(\mathrm{t}), 27.00(\mathrm{t})$, $32.04(\mathrm{t}), 33.34(\mathrm{t}), 105.03(\mathrm{~d}), 110.06(\mathrm{~d}), 140.88(\mathrm{~d}), 155.38(\mathrm{~s})$; mass spectrum: $202\left(6, \mathrm{M}^{+}\right), 81\left(100, \mathrm{M}-\left(\mathrm{CH}_{2}\right)_{3} \mathrm{Br}\right)$. Exact Mass calcd. for $\mathrm{C}_{8} \mathrm{H}_{11} \mathrm{BrO}$ : 201.9993 ; found: 201.9982 .

\section{7-(2-Furyl)-1-hepten-3-one (8)}

General procedure 1 was used to convert bromide $31(3.55 \mathrm{~g}$, $17.5 \mathrm{mmol})$ to the corresponding iodide $(4.16 \mathrm{~g}, 16.6 \mathrm{mmol})$ in $95 \%$ yield after distillation; bp $60-68^{\circ} \mathrm{C} / 0.08$ Torr; IR (neat) $\mathrm{cm}^{-1}$ : 
2936-2860(C-H); ${ }^{1} \mathrm{H}$ NMR (200 MHz): 1.64-2.01(m, 4H), 2.68(t, $2 \mathrm{H}, J=7.5 \mathrm{~Hz}), 3.21(\mathrm{t}, 2 \mathrm{H}, J=7.5 \mathrm{~Hz}), 6.02(\mathrm{dd}, 1 \mathrm{H}, J=$ $0.7 \mathrm{~Hz}, J=3.1 \mathrm{~Hz}$ ) 6.29 (dd, $1 \mathrm{H}, J=1.7 \mathrm{~Hz}, J=3.1 \mathrm{~Hz}$ ), $7.31(\mathrm{dd}, 1 \mathrm{H}, J=0.7 \mathrm{~Hz}, J=1.7 \mathrm{~Hz}) ;{ }^{13} \mathrm{C}$ NMR $(200 \mathrm{MHz})$ : $6.50(\mathrm{t}), \quad 26.80(\mathrm{t}), \quad 28.87(\mathrm{t}), \quad 32.78(\mathrm{t}), \quad 105.03(\mathrm{~d}), \quad 110.06(\mathrm{~d})$, 140.88(d), 155.35(s); mass spectrum: 250(22, $\left.\mathrm{M}^{+}\right), 123(30, \mathrm{M}-\mathrm{I})$, $81\left(100, \mathrm{M}-\left(\mathrm{CH}_{2}\right)_{3} \mathrm{I}\right)$. Exact Mass calcd. for $\mathrm{C}_{8} \mathrm{H}_{1 \mathrm{I}} \mathrm{IO}: 249.9851$; found: 249.9851 .

Using general procedure 2, the above iodide $(0.58 \mathrm{~g}, 2.32 \mathrm{mmol})$ was treated with tert-butyllithium $(3.0 \mathrm{~mL}, 5.09 \mathrm{mmol})$ and acrolein $(185 \mu \mathrm{L}, 2.78 \mathrm{mmol})$ to produce the corresponding allylic alcohol $(0.29 \mathrm{~g}, 1.61 \mathrm{mmol})$ as a clear, colourless oil in $69 \%$ yield after purification by flash chromatography $(7: 1)$ and distillation; bp $50-60^{\circ} \mathrm{C} / 0.05 \mathrm{Torr}$; 'H NMR $(200 \mathrm{MHz}): 1.39-1.75(\mathrm{~m}, 7 \mathrm{H})$, $2.64(\mathrm{t}, 2 \mathrm{H}, J=7.2 \mathrm{~Hz}), 4.11(\mathrm{br}, \mathrm{m}, 1 \mathrm{H}), 5.11(\mathrm{dt}, 1 \mathrm{H}, J=$ $1.2 \mathrm{~Hz}, J=1.2 \mathrm{~Hz}, J=10.3 \mathrm{~Hz}), 5.22(\mathrm{dt}, 1 \mathrm{H}, J=1.2 \mathrm{~Hz}, J=$ $1.2 \mathrm{~Hz}, J=17.2 \mathrm{~Hz}$ ), $5.91(\mathrm{ddd}, 1 \mathrm{H}, J=6.2 \mathrm{~Hz}, J=10.3 \mathrm{~Hz}$, $J=17.2 \mathrm{~Hz}), 5.99(\mathrm{dd}, 1 \mathrm{H}, J=0.7 \mathrm{~Hz}, J=3.0 \mathrm{~Hz}), 6.28(\mathrm{dd}$, $1 \mathrm{H}, J=1.9 \mathrm{~Hz}, J=3.0 \mathrm{~Hz}), 7.30(\mathrm{dd}, 1 \mathrm{H}, J=0.7 \mathrm{~Hz}, J=$ $1.9 \mathrm{~Hz}$ ).

The above allylic alcohol (133.9 $\mathrm{mg}, 0.74 \mathrm{mmol})$ was oxidized according to general procedure 3 to provide compound 8 $(113.3 \mathrm{~g}, 0.64 \mathrm{mmol})$ as a clear, colourless oil in $86 \%$ yield after purification by flash chromatography (20:1) and distillation; bp $80^{\circ} \mathrm{C} / 0.06 \mathrm{Torr} ;{ }^{1} \mathrm{H}$ NMR (200 MHz): $1.56-1.83$ (overlapping $\mathrm{m}$ ), 2.50-2.76(overlapping $\mathrm{m}$ ), 5.83(dd, $1 \mathrm{H}, J=2.5 \mathrm{~Hz}, J=$ $10.1 \mathrm{~Hz}), 5.98(\mathrm{dd}, 1 \mathrm{H}, J=0.7 \mathrm{~Hz}, J=3.0 \mathrm{~Hz}), 6.20(\mathrm{~d}$ of $\mathrm{ABq}$, $1 \mathrm{H}, J=2.5 \mathrm{~Hz}, J=17.5 \mathrm{~Hz}), 6.25(\mathrm{dd}, 1 \mathrm{H}, J=1.8 \mathrm{~Hz}, J=$ $3.0 \mathrm{~Hz}), 6.37(\mathrm{~d}$ of $\mathrm{ABq}, 1 \mathrm{H}, J=10.1 \mathrm{~Hz}, J=17.5 \mathrm{~Hz}), 7.30(\mathrm{dd}$, $1 \mathrm{H}, J=0.7 \mathrm{~Hz}, J=1.8 \mathrm{~Hz}$ ).

\section{4-Methyl-2-oxo-3-pentenenitrile (32)}

Freshly distilled acid chloride of 3-methyl-2-butenoic acid $(2.19 \mathrm{~g}, 18.5 \mathrm{mmol})$ was dissolved in dry acetonitrile $(15 \mathrm{~mL})$ under Ar. To this solution was added anhydrous $\mathrm{CuCN}$ ( 2 equiv.), and the suspension was heated to reflux (oil bath, $90^{\circ} \mathrm{C}$ ) for $30 \mathrm{~min}$, during which time a clear, brown solution was formed. The solution was cooled to room temperature, and the solvent was removed using a rotary evaporator (no heat), followed by an Ar back-purge. The desired compound was then distilled from the remaining brown solid, along with some acetonitrile. Conversion was confirmed by ${ }^{1} \mathrm{H}$ NMR, then the acyl cyanide 32 was used directly for the next step, bp $70-80^{\circ} \mathrm{C} / 20$ Torr; ${ }^{1} \mathrm{H}$ NMR (200 MHz): 2.07 and $2.30(\mathrm{~s}$, $3 \mathrm{H}$ each $), 6.25(\mathrm{~s}, 1 \mathrm{H})$.

\section{Methyl 3-(2-furyl)-3-methylbutanoate (33)}

A modification of the method of Ismail and Hoffmann (21) was used for the preparation of furan 33. The acyl cyanide $32(3.52 \mathrm{~g}$, $32.3 \mathrm{mmol})$ was dissolved in $\mathrm{CS}_{2}(120 \mathrm{~mL})$ under $\mathrm{Ar}$ and cooled in an ice bath. To this solution was added $\mathrm{AlCl}_{3}(1.08 \mathrm{~g}$, $8.10 \mathrm{mmol}$ ), resulting in a gummy precipitate. After stirring for $1 \mathrm{~h}$ at room temperature; furan $18(12.0 \mathrm{~mL}, 165.0 \mathrm{mmol})$ was added neat, and the reaction was stirred for $24 \mathrm{~h}$, after which time anhydrous methanol $(100 \mathrm{~mL})$ was added to the solution. The reaction was then stirred for an additional $24 \mathrm{~h}$. Evaporation of the $\mathrm{CS}_{2}$, followed by removal of methanol in vacuo, resulted in a viscous brown material. The crude product was taken up in ether $(200 \mathrm{~mL})$ and water $(100 \mathrm{~mL})$ followed by washing with $10 \%$ $\mathrm{Na}_{2} \mathrm{CO}_{3}(2 \times 100 \mathrm{~mL})$, then water $(2 \times 100 \mathrm{~mL})$, dried $\left(\mathrm{Na}_{2} \mathrm{SO}_{4}\right)$, and the ether removed in vacuo. Distillation of the crude brown oil provided 33 (27\% from 3-methyl-2-butenoic acid), bp $40-44^{\circ} \mathrm{C} /$ 0.03 Torr (lit. (21) bp $40^{\circ} \mathrm{C} / 0.03$ Torr); IR (neat) $\mathrm{cm}^{-1}: 1738$ $(\mathrm{C}=\mathrm{O}), 1077(\mathrm{C}-\mathrm{O}) ;{ }^{1} \mathrm{H}$ NMR $(200 \mathrm{MHz}) ; 1.40(\mathrm{~s}, 6 \mathrm{H}), 2.61(\mathrm{~s}$, $2 \mathrm{H}), 3.58(\mathrm{~s}, 3 \mathrm{H}), 6.01(\mathrm{dd}, 1 \mathrm{H}, J=0.8 \mathrm{~Hz}, J=3.2 \mathrm{~Hz}), 6.26(\mathrm{dd}$, $1 \mathrm{H}, J=1.9 \mathrm{~Hz}, J=3.2 \mathrm{~Hz}), 7.32(\mathrm{dd}, 1 \mathrm{H}, J=0.8 \mathrm{~Hz}, J=$ $1.9 \mathrm{~Hz}) ;{ }^{13} \mathrm{C}$ NMR $(50 \mathrm{MHz}): 2 \times 28.70(\mathrm{q}), 34.96(\mathrm{~s}), 45.73(\mathrm{t})$, 51.11(q), 103.12(d), 108.80(d), 140.66(d), 161.27(s), 171.58(s); mass spectrum: $182\left(58, \mathrm{M}^{*+}\right), 109\left(100, \mathrm{M}-\mathrm{CH}_{2} \mathrm{CO}_{2} \mathrm{Me}\right)$. Exact Mass calcd. for $\mathrm{C}_{10} \mathrm{H}_{14} \mathrm{O}_{3}$ : 182.0942; found: 182.0935 .

\section{Methyl 3-(2-(5-methylfuryl))-3-methylbutanoate (34)}

The acyl cyanide $32(1.82 \mathrm{~g}, 16.7 \mathrm{mmol})$ was dissolved in $\mathrm{CS}_{2}$ $(60 \mathrm{~mL})$ under Ar and cooled in an ice bath. To this solution was added $\mathrm{AlCl}_{3}(0.56 \mathrm{~g}, 4.2 \mathrm{mmol})$, resulting in a gummy precipitate. After stirring for $1 \mathrm{~h}$ at room temperature, 2-methylfuran 19 $(7.0 \mathrm{~mL}, 70.5 \mathrm{mmol})$ was added neat, and the reaction was stirred for $24 \mathrm{~h}$, after which time anhydrous methanol $(70 \mathrm{~mL})$ was added to the crimson solution. The reaction was then stirred for an additional $24 \mathrm{~h}$. Evaporation of the $\mathrm{CS}_{2}$, followed by removal of methanol in vacuo, resulted in a viscous brown material. The crude product was taken up in ether $(200 \mathrm{~mL})$ and water $(100 \mathrm{~mL})$, followed by washing with $10 \% \mathrm{Na}_{2} \mathrm{CO}_{3}(2 \times 100 \mathrm{~mL})$, then water $(2 \times 100 \mathrm{~mL})$, dried $\left(\mathrm{Na}_{2} \mathrm{SO}_{4}\right)$, and the ether removed in vacuo. Distillation of the crude brown oil provided $34(66 \%)$, bp 100$120^{\circ} \mathrm{C} / 15$ Torr; IR (neat) $\mathrm{cm}^{-1}: 1738(\mathrm{C}=\mathrm{O}), 1385,1365\left(\mathrm{CMe}_{2}\right)$ $1198(\mathrm{C}-\mathrm{O})$; ${ }^{1} \mathrm{H}$ NMR $(200 \mathrm{MHz}): 1.37(\mathrm{~s}, 6 \mathrm{H}), 2.25(\mathrm{~d}, 3 \mathrm{H}, J=$ $0.75 \mathrm{~Hz}), 2.58(\mathrm{~s}, 2 \mathrm{H}), 3.60(\mathrm{~s}, 3 \mathrm{H}), 5.87(\mathrm{~d}$ of $\mathrm{ABq}, 2 \mathrm{H}, J=$ $0.8 \mathrm{~Hz}, J=3.1 \mathrm{~Hz}) ;{ }^{13} \mathrm{C} \mathrm{NMR}(50 \mathrm{MHz}): 13.43(\mathrm{q}), 2 \times 26.69(\mathrm{q})$, 34.89(s), 45.82(6), 71.08(q), 103.64(d), 105.57(d), 150.33(s), $159.568 \mathrm{~s}), 171.77(\mathrm{~s})$; mass spectrum: $196\left(53, \mathrm{M}^{++}\right), 123(100$, $\mathrm{M}-\mathrm{CH}_{2} \mathrm{CO}_{2} \mathrm{Me}$ ). Anal. calcd. for $\mathrm{C}_{11} \mathrm{H}_{16} \mathrm{O}_{3}: \mathrm{C} 67.32, \mathrm{H} 8.22$; found: $\mathrm{C} 66.84, \mathrm{H} 8.18$

\section{3-(2-Furyl)-3-methyl-1-butanol (36)}

To $\mathrm{LiAlH}_{4}(0.51 \mathrm{~g}, 9.3 \mathrm{mmol})$, suspended in ether $(25 \mathrm{~mL})$ at $0^{\circ} \mathrm{C}$ under $\mathrm{Ar}$, was added dropwise a solution of freshly distilled $33(1.42 \mathrm{~g}, 7.8 \mathrm{mmol})$ in ether $(8 \mathrm{~mL})$. The reaction was warmed to room temperature and stirred for $12 \mathrm{~h}$. After cooling to $0^{\circ} \mathrm{C}$, the reaction was quenched using water $(0.5 \mathrm{~mL}), 15 \% \mathrm{NaOH}$ $(0.5 \mathrm{~mL})$, then water $(1.5 \mathrm{~mL})$. The white mixture was then filtered through Celite, and the solvent removed in vacuo to provide a clear, colourless oil. The oil was purified by flash chromatography $(1: 1)$, then distilled to yield compound $36(92 \%)$, bp $48^{\circ} \mathrm{C} /$ 0.07 Torr; IR (neat) $\mathrm{cm}^{-1}$ : $3333(\mathrm{OH}), 1385,1363\left(\mathrm{CMe}_{2}\right), 1077$, $1059(\mathrm{C}-\mathrm{O}) ;{ }^{1} \mathrm{H}$ NMR $(200 \mathrm{MHz}): 1.30(\mathrm{~s}, 6 \mathrm{H}), 1.51(\mathrm{~s}, 1 \mathrm{H})$, $1.89(\mathrm{t}, 2 \mathrm{H}, J=7.2 \mathrm{~Hz}), 3.54(\mathrm{t}, 2 \mathrm{H}, J=7.2 \mathrm{~Hz}), 5.99(\mathrm{dd}, 1 \mathrm{H}$, $J=0.9 \mathrm{~Hz}, J=3.2 \mathrm{~Hz}), 6.28(\mathrm{dd}, 1 \mathrm{H}, J=1.5 \mathrm{~Hz}, J=3.2 \mathrm{~Hz})$ 7.32(dd, $1 \mathrm{H}, J=0.9 \mathrm{~Hz}, J=1.5 \mathrm{~Hz}) ;{ }^{13} \mathrm{C} \mathrm{NMR}(50 \mathrm{MHz}): 2 \times$ $27.20(\mathrm{q}), 34.57(\mathrm{~s}), 44.61(\mathrm{t}), 59.92(\mathrm{t}), 103.24(\mathrm{~d}), 108.61(\mathrm{~d})$, 140.64(d), 163.32(s); mass spectrum: 154(35, $\left.\mathrm{M}^{*+}\right), 109(100$, $\left.\mathrm{M}-\left(\mathrm{CH}_{2}\right)_{2} \mathrm{OH}\right)$. Anal. calcd. for $\mathrm{C}_{9} \mathrm{H}_{11} \mathrm{O}_{2}: \mathrm{C} 70.09, \mathrm{H} 9.15$; found: C 69.66, H 9.00.

\section{3-(2-(5-Methylfuryl))-3-methyl-1-butanol (37)}

Compound $34(2.03 \mathrm{~g}, 10.4 \mathrm{mmol})$ was reduced to alcohol 37 (1.76 g, $10.1 \mathrm{mmol}$ ) by the method described for compound 36 in $97.5 \%$ yield after distillation, bp $60-70^{\circ} \mathrm{C} / 0.1$ Torr; IR (neat) $\mathrm{cm}^{-1}: 3334(\mathrm{OH}), 1384,1365\left(\mathrm{CMe}_{2}\right), 1059,1021(\mathrm{C}-\mathrm{O}) ;{ }^{1} \mathrm{H} \mathrm{NMR}$ $(200 \mathrm{MHz}): 1.28(\mathrm{~s}, 6 \mathrm{H}), 1.38(\mathrm{br} \mathrm{s}, 1 \mathrm{H}), 1.87(\mathrm{t}, 2 \mathrm{H}, J=7.1 \mathrm{~Hz})$, $2.26(\mathrm{~d}, 3 \mathrm{H}, J=0.8 \mathrm{~Hz}), 3.58(\mathrm{t}, 2 \mathrm{H}, J=7.1 \mathrm{~Hz}), 5.85(\mathrm{ABq}, 2 \mathrm{H})$; ${ }^{13} \mathrm{C}$ NMR(50 MHz): 13.45(q), $2 \times 27.22(\mathrm{q}), 34.36(\mathrm{~s}), 44.55(\mathrm{t})$, 59.97(t), 103.62(d), 105.51(d), 150.31(s), 160.46(s); mass spectrum: $168\left(60, \mathrm{M}^{++}\right), 123\left(100, \mathrm{M}-\left(\mathrm{CH}_{2}\right)_{2} \mathrm{OH}\right)$. Anal. calcd. for $\mathrm{C}_{10} \mathrm{H}_{16} \mathrm{O}_{2}$ : C 71.39, $\mathrm{H}$ 9.59; found: C 71.37, H 9.43.

\section{3-(2-Furyl)-1-iodo-3-methylbutane (38)}

Distilled compound $36(1.05 \mathrm{~g}, 6.80 \mathrm{mmol})$ was dissolved in dry $\mathrm{CH}_{2} \mathrm{Cl}_{2}(20 \mathrm{~mL})$, and cooled in an ice bath. To this solution was added $p$-toluenesulfonyl chloride $(2.59 \mathrm{~g}, 13.6 \mathrm{mmol})$ and DMAP $(1.66 \mathrm{~g}, 13.6 \mathrm{mmol})$, and the reaction was stirred for $12 \mathrm{~h}$. The mixture was poured into water $(50 \mathrm{~mL})$, then the organic layer was washed with $5 \% \mathrm{HCl}(2 \times 50 \mathrm{~mL})$, and water $(3 \times 50 \mathrm{~mL})$, then dried over $\mathrm{Na}_{2} \mathrm{SO}_{4}$. The solvent was removed in vacuo to yield a viscous clear oil, which was then purified by flash chromatography $(7: 1)$ to provide the tosylate of compound $36(2.03 \mathrm{~g}$, $6.58 \mathrm{mmol}$ ) in $98 \%$ yield. This decomposed when stored at room temperature; IR (neat) $\mathrm{cm}^{-1}: 3093-3035(\mathrm{C}-\mathrm{H}), 1364$, $1187\left(\mathrm{SO}_{2}\right.$ st. $) ;{ }^{1} \mathrm{H}$ NMR $(200 \mathrm{MHz}): 1.25(\mathrm{~s}, 6 \mathrm{H}), 1.97(\mathrm{t}, 2 \mathrm{H}, J=$ $7.3 \mathrm{~Hz}), 2.45(\mathrm{~s}, 3 \mathrm{H}), 3.93(\mathrm{t}, 2 \mathrm{H}, J=7.3 \mathrm{~Hz}), 5.91(\mathrm{dd}, 1 \mathrm{H}, J=$ $0.7 \mathrm{~Hz}, J=3.3 \mathrm{~Hz}), 6.22(\mathrm{dd}, 1 \mathrm{H}, J=1.8 \mathrm{~Hz}, J=3.3 \mathrm{~Hz}$ ), $7.25(\mathrm{~d}, 1 \mathrm{H}, J=0.7 \mathrm{~Hz}, J=1.8 \mathrm{~Hz}), 7.33$ and $7.74\left(\mathrm{AA}^{\prime} \mathrm{XX}^{\prime}, 4 \mathrm{H}\right)$; 
${ }^{13} \mathrm{C} \mathrm{NMR}(50 \mathrm{MHz}): 21.56(\mathrm{q}), 2 \times 27.02(\mathrm{q}), 34.61(\mathrm{~s}), 40.34(\mathrm{t})$, 87.95(t), 103.78(d), 109.76(d), 127.64(d), 129.71(d), 133.40(s), 144.52(s), $141.05(\mathrm{~d}), 160.92(\mathrm{~s})$; mass spectrum: $308\left(6, \mathrm{M}^{++}\right)$, $109\left(100, \mathrm{M}-\left(\mathrm{CH}_{2}\right)_{2} \mathrm{OTs}\right)$.

General procedure 1 was used to convert the above tosylate $(2.00 \mathrm{~g}, 6.5 \mathrm{mmol})$ to iodide $38(1.56 \mathrm{~g}, 5.91 \mathrm{mmol})$ in $91 \%$ yield after distillation, as a clear, colourless oil; bp $54-56^{\circ} \mathrm{C} / 0.06 \mathrm{Torr}$; IR (neat) $\mathrm{cm}^{-1}: 1386,1364\left(\mathrm{CMe}_{2}\right) ;{ }^{1} \mathrm{H}$ NMR $(200 \mathrm{MHz}): 1.28(\mathrm{~s}$, $6 \mathrm{H}), 2.26(\mathrm{~m}, 2 \mathrm{H}), 2.96(\mathrm{~m}, 2 \mathrm{H}), 6.00(\mathrm{dd}, 1 \mathrm{H}, J=0.7 \mathrm{~Hz}, J=$ $3.2 \mathrm{~Hz}), 6.27(\mathrm{dd}, 1 \mathrm{H}, J=1.8 \mathrm{~Hz}, J=3.2), 7.33(\mathrm{dd}, 1 \mathrm{H}, J=$ $0.7 \mathrm{~Hz}, J=1.8 \mathrm{~Hz}) ;{ }^{13} \mathrm{C} \mathrm{NMR}(50 \mathrm{MHz}): 0.35(\mathrm{t}), 2 \times 26.41(\mathrm{q})$, 38.14(s), 47.22(t), 103.92(d), 109.78(d), 141.13(d), 160.82(s); mass spectrum: $264\left(36, \mathrm{M}^{+}\right), 109\left(100, \mathrm{M}-\left(\mathrm{CH}_{2}\right)_{2} \mathrm{I}\right)$. Anal. calcd. for $\mathrm{C}_{9} \mathrm{H}_{13} \mathrm{IO}$ : $\mathrm{C} 40.93, \mathrm{H} 4.96$; found: $\mathrm{C} 40.93, \mathrm{H} 4.98$.

\section{3-(2-(5-Methylfuryl))-1-iodo-3-methylbutane (39)}

The tosylate of alcohol $37(2.70 \mathrm{~g}, 8.37 \mathrm{mmol})$ was prepared by the same method as above, in $87 \%$ yield after purification by flash chromatography $(9: 1)$ as a white, crystalline solid; mp $45-46^{\circ} \mathrm{C}$; IR $(\mathrm{KBr}) \mathrm{cm}^{-1}: 3087-3035(\mathrm{C}-\mathrm{H}), 1358$ and $1191\left(\mathrm{SO}_{2}\right)$; ${ }^{1} \mathrm{H} \mathrm{NMR}$ $(200 \mathrm{MHz}): 1.22(\mathrm{~s}, 6 \mathrm{H}), 1.94(\mathrm{t}, 2 \mathrm{H}, J=7.3 \mathrm{~Hz}), 2.20(\mathrm{~s}, 3 \mathrm{H})$, $2.46(\mathrm{~s}, 3 \mathrm{H}), 3.95(\mathrm{t}, 3 \mathrm{H}, J=7.3 \mathrm{~Hz}), 5.77(\mathrm{ABq}, 2 \mathrm{H}), 7.33$ and 7.75(AA'XX' $4 \mathrm{H}) ;{ }^{13} \mathrm{C}$ NMR (50 MHz): $13.41(\mathrm{q}), 21.55(\mathrm{q}), 2 \times$ 27.05(q), 34.45(s), 40.29(t), 66.14(t), 104.37(d), 105.54(d), 127.64(d), 129.70(d), 133.24(s), 144.49(s), 150.52(s), 159.07(s); mass spectrum: $322\left(6, \mathrm{M}^{\circ+}\right), 123\left(100, \mathrm{M}-\left(\mathrm{CH}_{2}\right)_{2} \mathrm{OTs}\right)$.

General procedure 1 was used to convert the above tosylate $(2.70 \mathrm{~g}, 8.37 \mathrm{mmol})$ to iodide $39(2.09 \mathrm{~g}, 7.51 \mathrm{mmol})$ in $90 \%$ yield after distillation, as a clear, colourless oil; bp $40-44^{\circ} \mathrm{C} / 0.04 \mathrm{Torr}$; IR (neat) $\mathrm{cm}^{-1}: 1385,1366\left(\mathrm{CMe}_{2}\right) ;{ }^{1} \mathrm{H}$ NMR $(200 \mathrm{MHz}): 1.25(\mathrm{~s}$, $6 \mathrm{H}), 2.23(\mathrm{~m}, 2 \mathrm{H}), 2.26(\mathrm{~d}, 3 \mathrm{H}, J=0.8 \mathrm{~Hz}), 2.99(\mathrm{~m}, 2 \mathrm{H}), 5.84(\mathrm{~m}$, 2H); ${ }^{13} \mathrm{C}$ NMR (50 MHz): 0.71(t), 13.52(q), 26.42(q), 37.98(s), 47.19(t), 104.50(d), 105.56(d), 150.53(s), 158.95(s); mass spectrum: $278\left(30, \mathrm{M}^{*+}\right), 123\left(100, \mathrm{M}-\left(\mathrm{CH}_{2}\right)_{2} \mathrm{I}\right)$. Exact Mass calcd. for $\mathrm{C}_{10} \mathrm{H}_{15} \mathrm{IO}$ : 278.0168; found: 278.0153 .

\section{6-(2-Furyl)-6-methyl-1-hepten-3-ol (40)}

Using general procedure 2, iodide 38 (250.1 $\mathrm{mg}, 0.947 \mathrm{mmol}$ ) was treated with tert-butyllithium $(1.16 \mathrm{~mL}, 1.96 \mathrm{mmol})$ and acrolein $(72 \mu \mathrm{L}, 1.08 \mathrm{mmol})$ to produce compound $40(89.1 \mathrm{mg}$, $0.458 \mathrm{mmol}$ ) as a clear, colourless oil in $48 \%$ yield after purification by flash chromatography $(5: 1)$; IR (neat) $\mathrm{cm}^{-1}: 3356(\mathrm{OH})$, $1644(\mathrm{C}=\mathrm{C}), \quad 1385,1363\left(\mathrm{gem}-\mathrm{CH}_{3}\right), \quad 1012(\mathrm{C}-\mathrm{O}) ;{ }^{\mathrm{l}} \mathrm{H} \quad \mathrm{NMR}$ $(200 \mathrm{MHz}): 1.26(\mathrm{~s}, 6 \mathrm{H}), 1.28-1.80(\mathrm{~m}, 5 \mathrm{H}), 3.99(\mathrm{q}, 1 \mathrm{H}, J=$ $6.5 \mathrm{~Hz}), 5.10(\mathrm{dt}, 1 \mathrm{H}, J=1.2 \mathrm{~Hz}, J=10.6 \mathrm{~Hz}), 5.18(\mathrm{dt}, 1 \mathrm{H}$, $J=1.2 \mathrm{~Hz}, J=17.1 \mathrm{~Hz}), 5.80(\mathrm{ddd}, 1 \mathrm{H}, J=7.0 \mathrm{~Hz}, J=$ $10.6 \mathrm{~Hz}, J=17.1 \mathrm{~Hz}), 5.98(\mathrm{dd}, 1 \mathrm{H}, J=0.8 \mathrm{~Hz}, J=3.1 \mathrm{~Hz})$, $6.25(\mathrm{dd}, 1 \mathrm{H}, J=1.7 \mathrm{~Hz}, J=3.1 \mathrm{~Hz}), 7.30(\mathrm{dd}, 1 \mathrm{H}, J=0.8 \mathrm{~Hz}$, $J=1.7 \mathrm{~Hz}) ;{ }^{13} \mathrm{C}$ NMR (50 MHz): 26.75(q), 26.87(q), 32.25(t), 35.42(s), 37.46(t), 73.41(d), 103.31(d), 109.63(d), 114.58(t), 140.62(d), 141.13(d), 162.59(s); mass spectrum: $194\left(9, \mathrm{M}^{*+}\right)$, $176\left(10, \mathrm{M}-\mathrm{H}_{2} \mathrm{O}\right), 161\left(37, \mathrm{M}-\mathrm{H}_{2} \mathrm{O}\right.$ and $\left.\mathrm{CH}_{3}\right), 109(100, \mathrm{M}-$ $\left.\left(\mathrm{CH}_{2}\right)_{2} \mathrm{CH}(\mathrm{OH}) \mathrm{CH}=\mathrm{CH}_{2}\right)$. Exact Mass calcd. for $\mathrm{C}_{12} \mathrm{H}_{18} \mathrm{O}_{2}$ : 194.1307; found: 194.1296.

\section{6-(2-Furyl)-2,6-dimethyl-1-hepten-3-ol (41)}

Using general procedure 2, iodide $38(201.1 \mathrm{mg}, 0.761 \mathrm{mmol})$ was treated with tert-butyllithium $(0.93 \mathrm{~mL}, 1.58 \mathrm{mmol})$ and methacrolein ( $72 \mu \mathrm{L}, 1.08 \mathrm{mmol}$ ) to produce compound $\mathbf{4 1}$ (108.7 $\mathrm{mg}, 0.522 \mathrm{mmol}$ ) as a clear, colourless oil in $68.6 \%$ yield after purification by flash chromatography $(5: 1)$ and distillation; bp $56^{\circ} \mathrm{C} / 0.035$ Torr; IR (neat) $\mathrm{cm}^{-1}: 3373(\mathrm{OH}), 1650(\mathrm{C}=\mathrm{C})$, $1384,1364\left(\mathrm{CMe}_{2}\right), 1013(\mathrm{C}-\mathrm{O}) ;{ }^{1} \mathrm{H}$ NMR $(200 \mathrm{MHz}): 1.26(\mathrm{~s}, 6 \mathrm{H})$, $1.29-1.75(\mathrm{~m}, 5 \mathrm{H}), 1.64(\mathrm{~s}, 3 \mathrm{H}), 3.96(\mathrm{br} \mathrm{t}, 1 \mathrm{H}, J=6.3 \mathrm{~Hz})$, 4.83(overlapping dd, $1 \mathrm{H}, J=1.5 \mathrm{~Hz}), 4.91(\mathrm{dd}, 1 \mathrm{H}, J=$ $1.6 \mathrm{~Hz}), 5.97(\mathrm{~d}, 1 \mathrm{H}, J=3.2 \mathrm{~Hz}), 6.26(\mathrm{dd}, 1 \mathrm{H}, J=1.8 \mathrm{~Hz}, J=$ $3.2 \mathrm{~Hz}), 7.31(\mathrm{~d}, 1 \mathrm{H}, J=1.8 \mathrm{~Hz}) ;{ }^{13} \mathrm{C}$ NMR $(50 \mathrm{MHz}): 17.29(\mathrm{q})$, 26.71(q), 26.98(q), 30.02(t), 35.45(s), 37.61(t), 76.27(d), 103.35(d), 109.67(d), 111.18(t), 140.64(d), 147.33(s), 162.66(s); mass spectrum: $208\left(13, \mathrm{M}^{+}\right), 190\left(4, \mathrm{M}-\mathrm{H}_{2} \mathrm{O}\right), 175\left(10, \mathrm{M}-\mathrm{H}_{2} \mathrm{O}\right.$ and $\mathrm{Me}), 109\left(100, \mathrm{M}-\left(\mathrm{CH}_{2}\right)_{2} \mathrm{CH}(\mathrm{OH}) \mathrm{C}(\mathrm{Me})=\mathrm{CH}_{2}\right)$. Anal. calcd. for $\mathrm{C}_{13} \mathrm{H}_{20} \mathrm{O}_{2}$ : C 74.96, $\mathrm{H}$ 9.68; found: $\mathrm{C} 74.93, \mathrm{H} 10.08$.

\section{(E)-7-(2-Furyl)-7-methyl-2-octen-4ol (42)}

Using general procedure 2, iodide $38(247.3 \mathrm{mg}, 0.936 \mathrm{mmol})$ was treated with tert-butyllithium $(1.21 \mathrm{~mL}, 2.06 \mathrm{mmol})$ and crotonaldehyde $(116 \mu \mathrm{L}, 1.40 \mathrm{mmol})$ to produce compound 42 ( $166.4 \mathrm{mg}, 0.800 \mathrm{mmol}$ ) as a clear, colourless oil in $84 \%$ yield after purification by flash chromatography $(5: 1)$ and distillation; IR (neat) $\mathrm{cm}^{-1}: 3350(\mathrm{OH}), 1673(\mathrm{C}=\mathrm{C}), 1077(\mathrm{C}-\mathrm{O})$; ${ }^{1} \mathrm{H}$ NMR $(200 \mathrm{MHz}): 1.26(\mathrm{~s}, 6 \mathrm{H}), 1.27-1.76(\mathrm{~m}, 5 \mathrm{H}), 1.69(\mathrm{dd}, 3 \mathrm{H}, J=$ $1.3 \mathrm{~Hz}, J=6.2 \mathrm{~Hz}), 3.93($ br. q, $1 \mathrm{H}, J=4.4 \mathrm{~Hz}), 5.44(\mathrm{dq}, 1 \mathrm{H}$, $J=1.8 \mathrm{~Hz}, J=6.9 \mathrm{~Hz}, J=15.3 \mathrm{~Hz}), 5.64(\mathrm{dq}, 1 \mathrm{H}, J=0.7 \mathrm{~Hz}$, $J=6.2 \mathrm{~Hz}, J=15.3 \mathrm{~Hz}), 5.96(\mathrm{dd}, 1 \mathrm{H}, J=0.9 \mathrm{~Hz}, J=$ $3.2 \mathrm{~Hz}), 6.26(\mathrm{dd}, 1 \mathrm{H}, J=1.8 \mathrm{~Hz}, J=3.2 \mathrm{~Hz}), 7.31(\mathrm{dd}, 1 \mathrm{H}$, $J=0.9 \mathrm{~Hz}, J=1.8 \mathrm{~Hz}) ;{ }^{13} \mathrm{C}$ NMR $(50 \mathrm{MHz}): 17.59(\mathrm{q}), 26.79(\mathrm{q})$, 26.91(q), 32.56(t), 35.50(s), 37.70(t), 73.41(d), 103.31(d), $109.65(\mathrm{~d}), 126.78(\mathrm{~d}), 134.28(\mathrm{~d}), 140.64(\mathrm{~d}), 162.74(\mathrm{~s})$; mass spectrum: $208\left(15, \mathrm{M}^{++}\right), 190\left(17, \mathrm{M}-\mathrm{H}_{2} \mathrm{O}\right), 175\left(43, \mathrm{M}-\mathrm{H}_{2} \mathrm{O}\right.$ and $\mathrm{Me}), 109\left(100, \mathrm{M}-\left(\mathrm{CH}_{2}\right)_{2} \mathrm{CH}(\mathrm{OH}) \mathrm{CH}=\mathrm{CHMe}\right)$. Anal. calcd. for $\mathrm{C}_{13} \mathrm{H}_{20} \mathrm{O}_{2}$ : C 74.96, $\mathrm{H}$ 9.68; found: $\mathrm{C} 74.85, \mathrm{H} 9.79$.

\section{(E)-7-(2-Furyl)-3,7-dimethyl-2-octen-4-ol (43)}

Using general procedure 2, iodide $38(346.1 \mathrm{mg}, 1.466 \mathrm{mmol})$ was treated with tert-butyllithium $(1.90 \mathrm{~mL}, 3.23 \mathrm{mmol})$ and tiglic aldehyde $(212 \mu \mathrm{L}, 2.20 \mathrm{mmol})$ to produce compound $\mathbf{4 3}$ (193.3 $\mathrm{mg}, 0.869 \mathrm{mmol}$ ) as a clear, colourless oil in $59.3 \%$ yield after purification by flash chromatography $(9: 1)$ and distillation; bp $68-72^{\circ} \mathrm{C} / 0.045$ Torr; IR (neat) $\mathrm{cm}^{-1}: 3353(\mathrm{OH}), 1671(\mathrm{C}=\mathrm{C})$, $1382,1363\left(\mathrm{CMe}_{2}\right), 1076(\mathrm{C}-\mathrm{O})$; 'H NMR (200 MHz): 1.25(s), $1.28-1.70(\mathrm{~m}, 5 \mathrm{H}), 1.52(\mathrm{dq}, 3 \mathrm{H}, J=1.0 \mathrm{~Hz}, J=2.2 \mathrm{~Hz}), 1.61(\mathrm{dq}$, $3 \mathrm{H}, J=0.9 \mathrm{~Hz}, J=6.7 \mathrm{~Hz}$ ), $3.88(\mathrm{br} \mathrm{t}, 1 \mathrm{H}, J=6.0 \mathrm{~Hz}$ ), 5.43 (qq, $1 \mathrm{H}, J=0.9 \mathrm{~Hz}, J=6.7 \mathrm{~Hz}), 5.96(\mathrm{dd}, 1 \mathrm{H}, J=0.9 \mathrm{~Hz}, J=$ $3.2 \mathrm{~Hz}), 6.26(\mathrm{dd}, 1 \mathrm{H}, J=1.9 \mathrm{~Hz}, J=3.2 \mathrm{~Hz}), 7.30(\mathrm{dd}, 1 \mathrm{H}$, $J=0.9 \mathrm{~Hz}, J=1.9 \mathrm{~Hz}) ;{ }^{13} \mathrm{C}$ NMR $(50 \mathrm{MHz}): 10.65(\mathrm{q}), 12.97(\mathrm{q})$, $26.67(\mathrm{q}), \quad 27.00(\mathrm{q}), \quad 29.96(\mathrm{t}), \quad 35.47(\mathrm{~s}), \quad 37.97(\mathrm{t}), \quad 78.37(\mathrm{~d})$, 103.29(d), 109.64(d), 120.94(d), 137.79(s), 140.60(d), 162.74(s); mass spectrum: $222\left(38, \mathrm{M}^{++}\right), 204\left(17, \mathrm{M}-\mathrm{H}_{2} \mathrm{O}\right), 189(30, \mathrm{M}-$ $\mathrm{H}_{2} \mathrm{O}$ and $\left.\mathrm{Me}\right), 109\left(100, \mathrm{M}-\left(\mathrm{CH}_{2}\right)_{2} \mathrm{CH}(\mathrm{OH}) \mathrm{CMe}=\mathrm{CHMe}\right)$. Exact mass calcd. for $\mathrm{C}_{14} \mathrm{H}_{22} \mathrm{O}_{2}: 222.1621$; found: 222.1609 .

\section{6-(2-(5-Methylfuryl))-6-methyl-1-hepten-3-ol (44)}

Using general procedure 2, iodide $39(297.9 \mathrm{mg}, 1.07 \mathrm{mmol})$ was treated with tert-butyllithium $(1.40 \mathrm{~mL}, 2.36 \mathrm{mmol})$ and acrolein (143 $\mu \mathrm{L}, 2.14 \mathrm{mmol})$ to produce compound 44 (154.5 $\mathrm{mg}$, $0.742 \mathrm{mmol}$ ) as a clear, colourless oil in $69 \%$ yield after purification by flash chromatography $(7: 1)$; IR(neat) $\mathrm{cm}^{-1}: 3408(\mathrm{OH})$, 1388, 1365( $\left.\mathrm{CMe}_{2}\right), 1019(\mathrm{C}-\mathrm{O})$; ' ${ }^{1} \mathrm{H}$ NMR $(300 \mathrm{MHz}): 1.20(\mathrm{~s}, 6 \mathrm{H})$, $1.29-1.66(\mathrm{~m}, 5 \mathrm{H}), 2.22(\mathrm{~s}, 3 \mathrm{H}), 3.98(\mathrm{br} \mathrm{q}, 1 \mathrm{H}, J=6.3 \mathrm{~Hz})$, $5.07(\mathrm{dt}, 1 \mathrm{H}, J=1.4 \mathrm{~Hz}, J=10.4 \mathrm{~Hz}), 5.17(\mathrm{dt}, 1 \mathrm{H}, J=1.2 \mathrm{~Hz}$, $J=17.2 \mathrm{~Hz}), 5.79(\mathrm{ABq}, 2 \mathrm{H}), 5.7(\mathrm{ddd}, 1 \mathrm{H}, J=6.2 \mathrm{~Hz}, J=$ $10.4 \mathrm{~Hz}, J=17.2 \mathrm{~Hz}) ;{ }^{13} \mathrm{C}$ NMR $(75 \mathrm{MHz}): 13.54(\mathrm{q}), 26.78(\mathrm{q})$, 26.89(q), 32.21(t), 37.37(t), 35.24(s), 73.53(d), 103.66(d), $105.38(\mathrm{~d}), \quad 114.63(\mathrm{t}), 141.08(\mathrm{~d}), 150.02(\mathrm{~s}), 160.76(\mathrm{~s})$; mass spectrum: $208\left(11, \mathrm{M}^{+}\right), 190\left(6, \mathrm{M}-\mathrm{H}_{2} \mathrm{O}\right), 175\left(19, \mathrm{M}-\mathrm{H}_{2} \mathrm{O}\right.$ and $\left.\mathrm{CH}_{3}\right), 123\left(100, \mathrm{M}-\left(\mathrm{CH}_{2}\right)_{2} \mathrm{CH}(\mathrm{OH}) \mathrm{CH}=\mathrm{CH}_{2}\right)$. Exact Mass calcd. for $\mathrm{C}_{13} \mathrm{H}_{20} \mathrm{O}_{2}$ : 208.1464; found: 208.1467

\section{6-(2-(5-Methylfuryl))-2,6-dimethyl-1-hepten-3-ol (45)}

Using general procedure 2, iodide $39(253.7 \mathrm{mg}, 0.912 \mathrm{mmol})$ was treated with tert-butyllithium $(1.20 \mathrm{~mL}, 2.01 \mathrm{mmol})$ and methacrolein $(151 \mu \mathrm{L}, 2.01 \mathrm{mmol})$ to produce compound 45 (113.4 mg, $0.510 \mathrm{mmol}$ ) as a clear, colourless oil in $56 \%$ yield after purification by flash chromatography $(7: 1)$ and distillation; bp $60^{\circ} \mathrm{C} / 0.065$ Torr; IR (neat) $\mathrm{cm}^{-7}: 3369(\mathrm{OH}), 1652(\mathrm{C}=\mathrm{C})$, 1384,1366(CMe $), 1021(\mathrm{C}-\mathrm{O})$; ${ }^{1} \mathrm{H}$ NMR $(300 \mathrm{MHz}): 1.20(\mathrm{~s}, 6 \mathrm{H})$, $1.29-1.59(\mathrm{~m}, 5 \mathrm{H}), 1.62(\mathrm{t}, 3 \mathrm{H}, J=1.1 \mathrm{~Hz}), 2.22(\mathrm{~d}, 3 \mathrm{H}, J=$ $0.8 \mathrm{~Hz}), 3.94(\mathrm{br} \mathrm{t}, 1 \mathrm{H}), 4.81(\mathrm{~m}, 1 \mathrm{H}, J=1.6 \mathrm{~Hz}), 4.88(\mathrm{~m}, 1 \mathrm{H})$, $5.80(\mathrm{ABq}, 2 \mathrm{H}) ;{ }^{13} \mathrm{C}$ NMR $(75 \mathrm{MHz}): 13.53(\mathrm{q}), 17.16(\mathrm{q}), 26.67(\mathrm{q})$, $27.02(\mathrm{q}), \quad 29.84(\mathrm{t}), \quad 37.38(\mathrm{t}), \quad 35.27(\mathrm{~s}), \quad 76.29(\mathrm{~d}), \quad 103.89(\mathrm{~d})$, 105.37(d), $111.33(\mathrm{t}), \quad 147.20(\mathrm{~s}), 149.98(\mathrm{~s}), 160.78(\mathrm{~s})$; mass 
spectrum (FI): 222.28. Exact Mass calcd. for $\mathrm{C}_{14} \mathrm{H}_{22} \mathrm{O}_{2}: 222.1620$; found: 222.1620 .

\section{(E)-7-(2-(5-Methylfuryl))-7-methyl-2-octen-4-ol (46)}

Using general procedure 2, iodide $39(270.4 \mathrm{mg}, 0.972 \mathrm{mmol})$ was treated with tert-butyllithium $(1.26 \mathrm{~mL}, 2.14 \mathrm{mmol})$ and crotonaldehyde $(16 \mathrm{l} \mu \mathrm{L}, 1.94 \mathrm{mmol}$ ) to produce compound 46 (194.3 $\mathrm{mg}, 0.874 \mathrm{mmol}$ ) as a clear, colourless oil in $90 \%$ yield after purification by flash chromatography $(9: 1)$ and distillation; IR (neat) $\mathrm{cm}^{-1}: 3457-3427(\mathrm{OH}), 1675(\mathrm{C}=\mathrm{C}), 1384,1367\left(\mathrm{CMe}_{2}\right)$, 1077(C-O); ${ }^{1} \mathrm{H}$ NMR $(300 \mathrm{MHz}): 1.20(\mathrm{~s}, 6 \mathrm{H}), 1.24-1.68(\mathrm{~m}, 5 \mathrm{H})$, $1.66(\mathrm{dd}, 3 \mathrm{H}, J=0.9 \mathrm{~Hz}, J=6.2 \mathrm{~Hz}), 2.22(\mathrm{~s}, 3 \mathrm{H}), 3.90(\mathrm{~m}, 1 \mathrm{H}$, $J=6.6 \mathrm{~Hz}), 5.43(\mathrm{dd}, 1 \mathrm{H}, J=0.9 \mathrm{~Hz}, J=6.6 \mathrm{~Hz}, J=$ $15.0 \mathrm{~Hz}), 5.57(\mathrm{dq}, 1 \mathrm{H}, J=6.2 \mathrm{~Hz}, J=15.0 \mathrm{~Hz}), 5.79(\mathrm{~s}, 2 \mathrm{H})$; ${ }^{13} \mathrm{C}$ NMR (75 MHz): 13.53(q), 17.62(q), 26.77(q), 26.89(q), $32.46(\mathrm{t}), 37.53(\mathrm{t}), 35.27(\mathrm{~s}), 73.44(\mathrm{~d}), 103.81(\mathrm{~d}), 105.37(\mathrm{~d})$, 126.76(d), 134.19(d), 149.97(s), 160.88(s); mass spectrum: 222(13, $\left.\mathrm{M}^{*+}\right), 123\left(100, \mathrm{M}-\left(\mathrm{CH}_{2}\right)_{2} \mathrm{CH}(\mathrm{OH}) \mathrm{CH}=\mathrm{CMe}\right)$. Anal. calcd. for $\mathrm{C}_{14} \mathrm{H}_{22} \mathrm{O}_{2}$ : C 75.63, $\mathrm{H}$ 9.97; found: C 75.25, H 9.99.

\section{6-(2-Furyl)-6-methyl-1-hepten-3-one (9)}

Compound $40(169.4 \mathrm{mg}, 0.872 \mathrm{mmol})$ was oxidized accord ing to general procedure 3 to provide compound $9(118.8 \mathrm{mg}$, $0.618 \mathrm{mmol}$ ) in $71 \%$ yield as a clear, colourless oil after purification by flash chromatography $(9 ; 1)$ and distillation; bp $50-56^{\circ} \mathrm{C} /$ 0.05 Torr; IR (neat) $\mathrm{cm}^{-1}: 1703$ and $1680(\mathrm{C}=0), 1615(\mathrm{C}=\mathrm{C}) ;{ }^{\mathrm{I}} \mathrm{H}$ NMR (200 MHz): 1.28(s, 6H), 1.88-1.96(m, 2H), 2.37-2.45(m, $2 \mathrm{H}), 5.77$ (ddd, $1 \mathrm{H}, J=1.5 \mathrm{~Hz}, J=10.6 \mathrm{~Hz}), 6.00(\mathrm{dd}, 1 \mathrm{H}, J=$ $3.2 \mathrm{~Hz}), 6.11(\mathrm{dd}, 1 \mathrm{H}, J=1.5 \mathrm{~Hz}, J=17.3 \mathrm{~Hz}), 6.28(\mathrm{dd}, 1 \mathrm{H}$, $J=10.6 \mathrm{~Hz}, J=17.3 \mathrm{~Hz}), 6.30(\mathrm{~m}, 1 \mathrm{H}), 7.32(\mathrm{dd}, 1 \mathrm{H}, J=$ $0.9 \mathrm{~Hz}) ;{ }^{13} \mathrm{C}$ NMR $(50 \mathrm{MHz}): 2 \times 26.46(\mathrm{q}), 35.09(\mathrm{~s}), 35.26(\mathrm{t})$, 35.56(t), 103.47(d), 109.44(d), 127.38(t), 136.12(d), 140.81(d), 161.56(s), 200.34(s); mass spectrum: 192(18, $\left.\mathrm{M}^{+}\right), 109(100$, $\left.\mathrm{M}-\left(\mathrm{CH}_{2}\right)_{2} \mathrm{C}(\mathrm{O}) \mathrm{CH}=\mathrm{CH}_{2}\right)$. Anal. calcd. for $\mathrm{C}_{13} \mathrm{H}_{18} \mathrm{O}_{2}: \mathrm{C} 75.69$, $\mathrm{H}$ 8.80; found: C 75.45, H 9.26.

\section{6-(2-Furyl)-2,6-dimethyl-1-hepten-3-one (10)}

Compound $41(83.1 \mathrm{mg}, 0.399 \mathrm{mmol})$ was oxidized according to general procedure 3 to provide compound $10(63.1 \mathrm{mg}$, $0.306 \mathrm{mmol}$ ) in $77 \%$ yield as a clear, colourless oil after purification by flash chromatography $(9: 1)$ and distillation; bp $50-56^{\circ} \mathrm{C} /$ 0.04 Torr; IR (neat) $\mathrm{cm}^{-1} 1679,1672(\mathrm{C}=\mathrm{O}), 1630(\mathrm{C}=\mathrm{C})$, $1385,1367\left(\mathrm{CMe}_{2}\right)$; ${ }^{1} \mathrm{H}$ NMR (200 MHz): 1.28(s, 6H), 1.83(dd, 3H, $J=0.8 \mathrm{~Hz}, J=1.4 \mathrm{~Hz}), 1.86-1.95(\mathrm{~m}, 2 \mathrm{H}), 2.46-2.54(\mathrm{~m}, 2 \mathrm{H})$, $5.70(\mathrm{~m}, 1 \mathrm{H}), 5.81(\mathrm{~m}, 1 \mathrm{H}), 5.99(\mathrm{dd}, 1 \mathrm{H}, J=0.8 \mathrm{~Hz}, J=$ $3.2 \mathrm{~Hz}), 6.26(\mathrm{dd}, 1 \mathrm{H}, J=1.8 \mathrm{~Hz}, J=3.2 \mathrm{~Hz}), 7.31(\mathrm{dd}, 1 \mathrm{H}$, $J=0.8 \mathrm{~Hz}, J=1.8 \mathrm{~Hz}) ;{ }^{13} \mathrm{C}$ NMR $(50 \mathrm{MHz}): 17.60(\mathrm{q}), 2 \times$ $26.79(\mathrm{q}), 33.35(\mathrm{t}), 35.44(\mathrm{~s}), 36.60(\mathrm{t}), 103.67(\mathrm{~d}), 109.72(\mathrm{~d})$, $124.09(\mathrm{t}), \quad 140.84(\mathrm{~d}), 144.40(\mathrm{~s}), 162.00(\mathrm{~s}), 202.03(\mathrm{~s})$; mass spectrum: $206\left(7, \mathrm{M}^{-+}\right), 109\left(100, \mathrm{M}-\left(\mathrm{CH}_{2}\right)_{2} \mathrm{C}(\mathrm{O}) \mathrm{C}(\mathrm{Me})=\mathrm{CH}_{2}\right)$. Exact Mass calcd. for $\mathrm{C}_{13} \mathrm{H}_{18} \mathrm{O}_{2}: 206.1307$; found: 206.1283 .

\section{(E)-7-(2-Furyl)-7-methyl-2-octen-4-one (11)}

Compound $42(166.4 \mathrm{mg}, 0.807 \mathrm{mmol})$ was oxidized according to general procedure 3 to provide compound $11(124.7 \mathrm{mg}$, $0.604 \mathrm{mmol}$ ) in $76 \%$ yield as a clear, colourless oil after purification by flash chromatography (20:1) and distillation; IR(neat): $3143-3013(\mathrm{C}-\mathrm{H}), \quad 2969-2871(\mathrm{C}-\mathrm{H}), \quad 1697, \quad 1673(\mathrm{C}=\mathrm{O})$, $1634(\mathrm{C}=\mathrm{C}), 1379,1365\left(\mathrm{CMe}_{2}\right) ;{ }^{1} \mathrm{H}$ NMR $(200 \mathrm{MHz}): 1.26(\mathrm{~s}, 6 \mathrm{H})$, $1.84(\mathrm{dd}, 3 \mathrm{H}, J=1.6 \mathrm{~Hz}, J=6.8 \mathrm{~Hz}), 1.84-1.92(\mathrm{~m}, 2 \mathrm{H}), 2.29$ 2.37(m, 2H), 5.97(dd, $1 \mathrm{H}, J=0.7 \mathrm{~Hz}, J=3.2 \mathrm{~Hz}), 6.04(\mathrm{dq}, 1 \mathrm{H}$, $J=1.6 \mathrm{~Hz}, J=15.8 \mathrm{~Hz}), 6.25(\mathrm{dd}, 1 \mathrm{H}, J=1.9 \mathrm{~Hz}, J=$ $3.2 \mathrm{~Hz}), 6.72(\mathrm{dq}, 1 \mathrm{H}, J=6.8 \mathrm{~Hz}, J=15.8 \mathrm{~Hz}), 7.29(\mathrm{dd}, 1 \mathrm{H}$, $J=0.7 \mathrm{~Hz}, J=1.8 \mathrm{~Hz}) ;{ }^{13} \mathrm{C}$ NMR $(50 \mathrm{MHz}): 17.96(\mathrm{q}), 2 \times$ 26.68(q), 35.31(s), 35.76(t), 35.96(t), 103.59(d), 109.62(d), 131.72(d), 140.75(d), 142.00(d), 161.89(s), 200.22(s); mass spectrum: $206\left(36, \mathrm{M}^{+}\right), 109\left(100, \mathrm{M}-\left(\mathrm{CH}_{2}\right)_{2} \mathrm{C}(\mathrm{O}) \mathrm{CH}=\mathrm{CHMe}\right)$. Exact Mass calcd. for $\mathrm{C}_{13} \mathrm{H}_{18} \mathrm{O}_{2}: 206.1307$; found: 206.1307.

(E)-7-(2-Furyl)-3,7-dimethyl-2-octen-4-one (12)

Compound 43 (153.2 $\mathrm{mg}, 0.689 \mathrm{mmol})$ was oxidized according to general procedure 3 to provide compound 12 (144.2 mg,
$0.654 \mathrm{mmol}$ ) in $95 \%$ yield as a clear, colourless oil after purification by flash chromatography $(20: 1)$ and distillation; bp $80-90^{\circ} \mathrm{C} /$ 0.06 Torr; IR(neat) $\mathrm{cm}^{-1}: \quad 1667(\mathrm{C}=\mathrm{O}), \quad 1644(\mathrm{C}=\mathrm{C})$, $1379,1363\left(\mathrm{CMe}_{2}\right) ;{ }^{1} \mathrm{H}$ NMR $(200 \mathrm{MHz}): 1.28(\mathrm{~s}, 6 \mathrm{H}), 1.74(\mathrm{~d}, 3 \mathrm{H}$, $J=1.0 \mathrm{~Hz}), 1.82(\mathrm{~d}, 3 \mathrm{H}, J=6.9 \mathrm{~Hz}), 1.85-1.94(\mathrm{~m}, 2 \mathrm{H}), 2.42-$ $2.5(\mathrm{~m}, 2 \mathrm{H}), 5.99(\mathrm{dd}, 1 \mathrm{H}, J=0.7 \mathrm{~Hz}, J=3.2 \mathrm{~Hz}), 6.20(\mathrm{dd}, 1 \mathrm{H}$, $J=1.8 \mathrm{~Hz}, J=3.2 \mathrm{~Hz}), 6.58(\mathrm{~m}, 1 \mathrm{H}, J=1.0 \mathrm{~Hz}, J=6.9 \mathrm{~Hz})$, 7.32(dd, $1 \mathrm{H}, J=0.8 \mathrm{~Hz}, J=1.8 \mathrm{~Hz}) ;{ }^{13} \mathrm{C}$ NMR $(50 \mathrm{MHz})$ : $10.98(\mathrm{q}), 14.59(\mathrm{q}), 2 \times 26.77(\mathrm{q}), 32.95(\mathrm{t}), 36.87(\mathrm{t}), 35.44(\mathrm{~s})$, 103.60 (d), 109.89(d), 136.66(d), 138.05(s), 140.77(d), 162.10(s), 201.76(s); mass spectrum: 220 $\left(45, \mathrm{M}^{++}\right), 109(100, \mathrm{M}-$ $\left.\left(\mathrm{CH}_{2}\right)_{2} \mathrm{C}(\mathrm{O}) \mathrm{CMe}=\mathrm{CHMe}\right)$. Exact Mass calcd. for $\mathrm{C}_{14} \mathrm{H}_{20} \mathrm{O}_{2}$ : 220.1464 ; found: 220.1461 .

\section{6-(2-(5-Methylfuryl))-6-methyl-1-hepten-3-one (13)}

Compound $44(122.0 \mathrm{mg}, 0.586 \mathrm{mmol})$ was oxidized according to general procedure 3 to provide compound $13(80.9 \mathrm{mg}$, $0.392 \mathrm{mmol}$ ) in $67 \%$ yield as a clear, colourless oil after purification by flash chromatography $(9: 1)$ and distillation; bp $52-58^{\circ} \mathrm{C} /$ 0.03 Torr; IR (neat) $\mathrm{cm}^{-1}: 1682(\mathrm{C}=\mathrm{O}), 1614(\mathrm{C}=\mathrm{C})$; 'H NMR $(300 \mathrm{MHz}): 1.22(\mathrm{~s}, 6 \mathrm{H}), 1.84-1.89(\mathrm{~m}, 2 \mathrm{H}), 2.22(\mathrm{~d}, 3 \mathrm{H}, J=$ $0.9 \mathrm{~Hz}), 2.37-2.42(\mathrm{~m}, 2 \mathrm{H}), 5.75(\mathrm{dd}, 1 \mathrm{H}, J=1.3 \mathrm{~Hz}, J=$ $10.4 \mathrm{~Hz}), 5.80(\mathrm{dq}, 1 \mathrm{H}, J=1.0 \mathrm{~Hz}, J=3.0 \mathrm{~Hz}), 5.83(\mathrm{~d}, 1 \mathrm{H}$, $J=3.0 \mathrm{~Hz}), 6.10(\mathrm{dd}, 1 \mathrm{H}, J=1.3 \mathrm{~Hz}, J=17.6 \mathrm{~Hz}), 6.26(\mathrm{dd}$, $1 \mathrm{H}, J=10.4 \mathrm{~Hz}, J=17.6 \mathrm{~Hz}) ;{ }^{13} \mathrm{C}$ NMR $(75 \mathrm{MHz}): 13.55(\mathrm{q})$, $2 \times 26.62(\mathrm{q}), 35.23(\mathrm{~s}), 35.62(\mathrm{t}), 35.79(\mathrm{t}), 104.34(\mathrm{~d}), 105.47(\mathrm{~d})$, $127.76(\mathrm{t}), \quad 136.44(\mathrm{~d}), \quad 150.37(\mathrm{~s}), \quad 159.98(\mathrm{~s}), 201.01(\mathrm{~s}) ;$ mass spectrum: $206\left(24, \mathrm{M}^{++}\right), 123\left(100, \mathrm{M}-\left(\mathrm{CH}_{2}\right)_{2} \mathrm{C}(\mathrm{O}) \mathrm{CH}=\mathrm{CH}_{2}\right)$. Exact Mass calcd. for $\mathrm{C}_{13} \mathrm{H}_{18} \mathrm{O}_{2}: 206.1307$; found: 206.1294 .

\section{6-(2-(5-Methylfuryl))-2,6-dimethyl-1-hepten-3-one (14)}

Compound $45(90.2 \mathrm{mg}, 0.406 \mathrm{mmol})$ was oxidized according to general procedure 3 to provide compound $14(88.3 \mathrm{mg}$, $0.401 \mathrm{mmol}$ ) in $99 \%$ yield as a clear, colourless oil after purification by flash chromatography $(9: 1)$ and distillation; bp $60^{\circ} \mathrm{C} /$ 0.055 Torr; $\mathrm{mp} 27.5-30^{\circ} \mathrm{C}$; IR (neat) $\mathrm{cm}^{-1}$ : $1677(\mathrm{C}=\mathrm{O})$, $1625(\mathrm{C}=\mathrm{C}) ;{ }^{1} \mathrm{H}$ NMR $(300 \mathrm{MHz}): 1.22(\mathrm{~s}, 6 \mathrm{H}), 1.81(\mathrm{~d}, 3 \mathrm{H}, J=$ $1.1 \mathrm{~Hz}), 1.82-1.87(\mathrm{~m}, 2 \mathrm{H}), 2.21(\mathrm{~d}, 3 \mathrm{H}, J=0.8 \mathrm{~Hz}), 2.45-$ $2.51(\mathrm{~m}, 2 \mathrm{H}), 5.68(\mathrm{~m}, 1 \mathrm{H}), 5.80(\mathrm{~m}, 3 \mathrm{H}) ;{ }^{13} \mathrm{C}$ NMR $(75 \mathrm{MHz})$ : 13.53(q), 17.67(q), $2 \times 26.63(\mathrm{q}), 33.43(\mathrm{t}), 36.56(\mathrm{t}), 35.30(\mathrm{~s})$, $104.28(\mathrm{~d}), 105.47(\mathrm{~d}), 124.29(\mathrm{t}), 144.31(\mathrm{~s}), 150.29(\mathrm{~s}), 160.1(\mathrm{~s})$, 202.39(s); mass spectrum: 220(18, $\left.\mathrm{M}^{*+}\right), 123(100, \mathrm{M}-$ $\left.\left(\mathrm{CH}_{2}\right)_{2} \mathrm{C}(\mathrm{O}) \mathrm{C}(\mathrm{Me})=\mathrm{CH}_{2}\right)$. Exact Mass calcd. for $\mathrm{C}_{14} \mathrm{H}_{20} \mathrm{O}_{2}$ : 220.1463; found: 220.1473 .

\section{(E)-7-(2-(5-Methylfuryl))-7-methyl-2-octen-4-one (15)}

Compound 46 (199.8 mg, $0.899 \mathrm{mmol}$ ) was oxidized according to general procedure 3 to provide compound 15 (169.3 mg, $0.768 \mathrm{mmol}$ ) in $86 \%$ yield as a clear, colourless oil after purification by flash chromatography $(20: 1)$ and distillation, bp $62-64^{\circ} \mathrm{C} /$ 0.055 Torr; IR (neat) $\mathrm{cm}^{-1}: 3034(\mathrm{CH}), 2988-2869(\mathrm{CH}), 1697$, $1674(\mathrm{C}=\mathrm{O}), 1634(\mathrm{C}=\mathrm{C}), 1385,1366(\mathrm{gem}-\mathrm{Me}) ;{ }^{1} \mathrm{H}$ NMR $(300$ $\mathrm{MHz}): 1.25(\mathrm{~s}, 6 \mathrm{H}), 1.82-1.91$ (overlapping $\mathrm{m}, 5 \mathrm{H}), 2.25(\mathrm{~s}, 3 \mathrm{H})$, $2.32-2.40(\mathrm{~m}, 2 \mathrm{H}), 5.84(\mathrm{~m}, 2 \mathrm{H}), 6.06(\mathrm{dq}, 1 \mathrm{H}, J=1.6 \mathrm{~Hz}, J=$ $15.7 \mathrm{~Hz}), 6.74(\mathrm{dq}, 1 \mathrm{H}, J=6.8 \mathrm{~Hz}, J=15.7 \mathrm{~Hz}) ;{ }^{13} \mathrm{C} \mathrm{NMR}$ $(75 \mathrm{MHz}): 13.56(\mathrm{q}), 18.16(\mathrm{q}), 2 \times 26.82(\mathrm{q}), 35.27(\mathrm{~s}), 35.95(\mathrm{t})$, $36.01(\mathrm{t}), 104.30(\mathrm{~d}), 105.45(\mathrm{~d}), 131.84(\mathrm{~d}), 142.22(\mathrm{~d}), 150.29(\mathrm{~s})$, 160.12(s), 200.66(s); mass spectrum: 220(16, $\left.\mathrm{M}^{++}\right), 123(100$, $\left.\mathrm{M}-\left(\mathrm{CH}_{2}\right)_{2} \mathrm{C}(\mathrm{O}) \mathrm{CH}=\mathrm{CHMe}\right)$. Exact Mass calcd. for $\mathrm{C}_{14} \mathrm{H}_{20} \mathrm{O}_{2}$ : 220.1463 ; found: 220.1462 .

\section{6-(2-Furyl)-6-methyl-1-(trimethylsilyl)-1-heptyn-3-one (16)}

Using general procedure 2, iodide $38(285.1 \mathrm{mg}, 1.08 \mathrm{mmol})$ was treated with tert-butyllithium $(1.40 \mathrm{~mL}, 2.37 \mathrm{mmol})$ and aldehyde $47(202.6 \mathrm{mg}, 1.61 \mathrm{mmol})$ to produce the corresponding allynic alcohol $(187.6 \mathrm{~g}, 0.71 \mathrm{mmol})$ as a clear, colourless oil in $66 \%$ yield after purification by flash chromatography $(7: 1)$ and distillation; bp $80-88^{\circ} \mathrm{C} / 0.065$ Torr; IR (neat) $\mathrm{cm}^{-1}$ : $3380(\mathrm{O}-\mathrm{H})$, $1055(\mathrm{C}-\mathrm{O})$; ${ }^{1} \mathrm{H}$ NMR $(200 \mathrm{MHz}): 0.18(\mathrm{~s}, 9 \mathrm{H}), 1.28(\mathrm{~s}, 6 \mathrm{H}), 1.47-$ 1.81 (overlapping $\mathrm{m}, 5 \mathrm{H}), 4.27$ (br q, $1 \mathrm{H}, J=5.9 \mathrm{~Hz}$ ), $5.99(\mathrm{dd}$, $1 \mathrm{H}, J=0.8, J=3.2 \mathrm{~Hz}), 6.27(\mathrm{dd}, 1 \mathrm{H}, J=1.9, J=3.2 \mathrm{~Hz})$, 
$7.31(\mathrm{dd}, 1 \mathrm{H}, J=0.8, J=1.9 \mathrm{~Hz}) ;{ }^{13} \mathrm{C}$ NMR $(50 \mathrm{MHz}):-0.14(\mathrm{q})$, $2 \times 26.88(\mathrm{q}), 33.24(\mathrm{t}), 37.22(\mathrm{t}), 63.12(\mathrm{~d}), 89.40(\mathrm{~s}), 106.80(\mathrm{~s})$, 103.37(d), 109.66(d), 140.73(d), 162.43(s); mass spectrum: 264(4, $\left.\mathrm{M}^{++}\right), \quad 249(15, \mathrm{M}-\mathrm{Me}), \quad 109\left(100, \mathrm{M}-\left(\mathrm{CH}_{2}\right)_{2} \mathrm{CH}(\mathrm{OH})-\right.$ $\mathrm{C} \equiv \mathrm{CSiMe}_{3}$ ). Exact Mass calcd. for $\mathrm{C}_{15} \mathrm{H}_{24} \mathrm{O}_{2} \mathrm{Si}$ : 264.1546: found: 264.1535 .

The above alcohol ( $123.3 \mathrm{mg}, 0.47 \mathrm{mmol}$ ) was oxidized according to general procedure 3 to provide compound $\mathbf{1 6}$ (116.6 mg, $0.44 \mathrm{mmol}$ ) as a clear, colourless oil in $95 \%$ yield after purification by flash chromatography $(20: 1)$ and distillation; bp 68 $74^{\circ} \mathrm{C} / 0.04$ Torr: IR (neat) $\mathrm{cm}^{-1}: 2151(\mathrm{C} \equiv \mathrm{C}), 1679(\mathrm{C}=\mathrm{O}) ;{ }^{1} \mathrm{H}$ NMR $(200 \mathrm{MHz}): 0.25(\mathrm{~s}, 9 \mathrm{H}), 1.28(\mathrm{~s}, 6 \mathrm{H}), 1.90-2.04(\mathrm{~m}, 2 \mathrm{H})$, $2.33-2.50(\mathrm{~m}, 2 \mathrm{H}), 6.00(\mathrm{dd}, 1 \mathrm{H}, J=0.8$ and $3.0 \mathrm{~Hz}), 6.29(\mathrm{dd}$, $1 \mathrm{H}, J=1.7$ and $3.0 \mathrm{~Hz}), 7.33(\mathrm{dd}, 1 \mathrm{H}, J=0.8$ and $1.7 \mathrm{~Hz}) ;{ }^{13} \mathrm{C}$ $\operatorname{NMR}(50 \mathrm{MHz}): 0.80(\mathrm{q}), 2 \times 26.73(\mathrm{q}), 35.20(\mathrm{~s}), 35.38(\mathrm{t})$, 41.34(t), 97.50(s), 101.93(s), 103.82(d), 109.70(d), 140.99(d), 161.47(s), 187.51(s); mass spectrum: $262\left(5, \mathrm{M}^{++}\right), 247(15, \mathrm{M}-$ $\mathrm{Me}), 109\left(100, \mathrm{M}-\left(\mathrm{CH}_{2}\right)_{2} \mathrm{C}(\mathrm{O}) \mathrm{C} \equiv \mathrm{CSiMe}_{3}\right)$. Exact Mass calcd. for $\mathrm{C}_{15} \mathrm{H}_{22} \mathrm{O}_{2} \mathrm{Si}$ : 262.1389; found: 262.1385.

\section{7-(2-Furyl)-7-methyl-2-octyn-4-one (17)}

Using general procedure 2 , iodide $38(601.5 \mathrm{mg}, 2.28 \mathrm{mmol})$ was treated with tert-butyllithium $(2.95 \mathrm{~mL}, 5.01 \mathrm{mmol})$ and aldehyde $48(620.1 \mathrm{mg}, 9.12 \mathrm{mmol})$ to produce the corresponding alcohol $(378.8 \mathrm{~g}, 1.84 \mathrm{mmol})$ as a clear, colourless oil in $81 \%$ yield after purification by flash chromatography $(7: 1)$ and distillation; bp 70 $80^{\circ} \mathrm{C} / 0.045$ Torr; IR (neat) $\mathrm{cm}^{-1}: 3520(\mathrm{OH}), 1054(\mathrm{C}-\mathrm{O}) ;{ }^{1} \mathrm{H}$ NMR $(200 \mathrm{MHz}): 1.28(\mathrm{~s}, 6 \mathrm{H}), 1.44-1.83$ (overlapping $\mathrm{m}, 5 \mathrm{H})$, $1.84(\mathrm{~d}, 3 \mathrm{H}, J=2.2 \mathrm{~Hz}), 4.22-4.25(\mathrm{br} \mathrm{m}, 1 \mathrm{H}), 5.98(\mathrm{dd}, 1 \mathrm{H}$, $J=0.7 \mathrm{~Hz}, J=3.2 \mathrm{~Hz}), 6.26(\mathrm{dd}, 1 \mathrm{H}, J=1.8 \mathrm{~Hz}, J=3.2 \mathrm{~Hz})$, $7.31(\mathrm{dd}, 1 \mathrm{H}, J=0.7 \mathrm{~Hz}, J=1.8 \mathrm{~Hz}) ;{ }^{13} \mathrm{C} \operatorname{NMR}(50 \mathrm{MHz})$ : $3.37(\mathrm{q}), 2 \times 26.74(\mathrm{q}), 33.46(\mathrm{t}), 35.26(\mathrm{t}), 37.19(\mathrm{t}), 62.76(\mathrm{~d})$, $80.37(\mathrm{~s}), 80.73(\mathrm{~s}), 103.24(\mathrm{~d}), 109.56(\mathrm{~d}), 140.59(\mathrm{~d}), 162.42(\mathrm{~s})$; mass spectrum: $206\left(10, \mathrm{M}^{+}\right), 109\left(100, \mathrm{M}-\left(\mathrm{CH}_{2}\right)_{2} \mathrm{CH}(\mathrm{OH})-\right.$ $\mathrm{C} \equiv \mathrm{CCH}_{3}$ ). Exact Mass calcd. for $\mathrm{C}_{13} \mathrm{H}_{18} \mathrm{O}_{2}: 206.1307$; found: 206.1307 .

The above alcohol (213.1 $\mathrm{mg}, 1.03 \mathrm{mmol}$ ) was oxidized according to general procedure 3 to provide compound 17 (200.9 mg, $0.98 \mathrm{mmol}$ ) as a clear, colourless oil in $95 \%$ yield after purification by flash chromatography $(20: 1)$ and distillation; bp 76$80^{\circ} \mathrm{C} / 0.08$ Torr. IR (neat) $\mathrm{cm}^{-1}: 2218(\mathrm{C} \equiv \mathrm{C}), 1673(\mathrm{C}=\mathrm{O}) ;{ }^{\prime} \mathrm{H}$ NMR $(200 \mathrm{MHz}): 1.27(\mathrm{~s}, 6 \mathrm{H}), 1.90-1.98(\mathrm{~m}, 2 \mathrm{H}), 2.00(\mathrm{~s}, 3 \mathrm{H})$, $2.34(\mathrm{~m}, 2 \mathrm{H}), 5.99(\mathrm{dd}, 1 \mathrm{H}, J=0.8 \mathrm{~Hz}, J=3.2 \mathrm{~Hz}), 6.27(\mathrm{dd}, 1 \mathrm{H}$, $J=1.9 \mathrm{~Hz}, J=3.2 \mathrm{~Hz}), 7.32(\mathrm{dd}, 1 \mathrm{H}, J=0.8 \mathrm{~Hz}, J=1.9 \mathrm{~Hz})$; ${ }^{13} \mathrm{C}$ NMR $(50 \mathrm{MHz}): 3.96(\mathrm{q}), 2 \times 26.70(\mathrm{q}), 35.22(\mathrm{~s}), 35.54(\mathrm{~d})$, $41.44(\mathrm{~d}), 80.18(\mathrm{~s}), 89.78(\mathrm{~s}), 103.75(\mathrm{~d}), 109.70(\mathrm{~d}), 140.96(\mathrm{~d})$, 161.61(s), 187.86(s); mass spectrum: 204(11, $\left.\mathrm{M}^{++}\right), 109(100$, $\left.\mathrm{M}-\left(\mathrm{CH}_{2}\right)_{2} \mathrm{C}(\mathrm{O}) \mathrm{C} \equiv \mathrm{CCH}_{3}\right)$ Exact Mass calcd. for $\mathrm{C}_{13} \mathrm{H}_{16} \mathrm{O}_{2}$ : 204. 1150 ; found: 204.1151.

$(6 \alpha, 8 \alpha-\mathrm{H})-11$-Oxatricyclo[6.2.1.0. $\left.0^{1.6}\right]$ undec-9-en-5-one (49)

General procedure 5 was used for the Lewis acid-mediated IMDAF reaction of compound 1 . Thus, enone 1 (54.1 $\mathrm{mg}$, $0.329 \mathrm{mmol})$ was treated with $\mathrm{MeAlCl}_{2}(362 \mu \mathrm{L}, 0.362 \mathrm{mmol})$ at $-78^{\circ} \mathrm{C}$ for $1 \mathrm{~h}$ to provide adduct $49(54.0 \mathrm{mg}, 0.329 \mathrm{mmol})$ in $99 \%$ yield as white, crystalline solid; mp $25-27^{\circ} \mathrm{C}$; IR $(\mathrm{KBr}) \mathrm{cm}^{-1}$ : $1708(\mathrm{C}=\mathrm{O}), 1153(\mathrm{C}-\mathrm{O})$; ${ }^{1} \mathrm{H}$ NMR $(400 \mathrm{MHz}): 1.47(\mathrm{dd}, 1 \mathrm{H}$, $\left.J_{7 \alpha .6 \alpha}=8.2 \mathrm{~Hz}, J_{g^{\prime} m}=11.8 \mathrm{~Hz}, \mathrm{H}-7 \alpha\right), 1.79-2.08(\mathrm{~m}, 2 \mathrm{H})$, $2.28\left(\mathrm{dd}, 1 \mathrm{H}, J_{6 \alpha, 7 \beta}=3.0 \mathrm{~Hz}, J_{6 \alpha, 7 \alpha}=8.2 \mathrm{~Hz}, \mathrm{H}-6 \alpha\right.$ ), 2.49 (ddd, $\left.J_{7 \beta, 6 \alpha}=3.0 \mathrm{~Hz}, J_{7 \beta, 8}=4.8 \mathrm{~Hz}, J_{g e m}=11.8 \mathrm{~Hz}, \mathrm{H}-7 \beta\right), 2.20$ $2.58(\mathrm{~m}, 4 \mathrm{H}), 4.89\left(\mathrm{dd}, 1 \mathrm{H}, J_{8,9}=1.6 \mathrm{~Hz}, J_{8,7 \beta}=4.8 \mathrm{~Hz}, \mathrm{H}-8\right)$, $6.27\left(\mathrm{~d}, 1 \mathrm{H}, J_{10,9}=5.7 \mathrm{~Hz}, \mathrm{H}-10\right), 6.42\left(\mathrm{dd}, 1 \mathrm{H}, J_{8,9}=1.7 \mathrm{~Hz}\right.$, $\left.J_{9,10}=5.7 \mathrm{~Hz}, \mathrm{H}-9\right) ;{ }^{13} \mathrm{C} \mathrm{NMR}(50 \mathrm{MHz}): 21.73,28.17,29.10$ and $41.66(t, C-2, C-3, C-4$ and C-7), 50.30(d, C-6), 78.08(d, C-8), $90.40(\mathrm{~s}, \mathrm{C}-1), 136.92$ and $138.20(\mathrm{~d}, \mathrm{C}-9$ and $\mathrm{C}-10), 209.30(\mathrm{~s}$, $\mathrm{C}-5)$; mass spectrum (FI): $164\left(100, \mathrm{M}^{+}\right)$. Exact Mass calcd. for $\mathrm{C}_{10} \mathrm{H}_{12} \mathrm{O}_{2}: 164.0838$; found: 164.0819 .

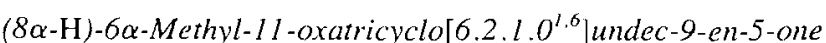
(50)

General procedure 5 was used to perform the Lewis acidmediated reaction of compound 2 . Thus, enone $2(66.6 \mathrm{mg}$, $0.374 \mathrm{mmol})$ was treated with $\mathrm{MeAlCl}_{2}(411 \mu \mathrm{L}, 0.411 \mathrm{mmol})$ at $-78^{\circ} \mathrm{C}$ for $2.5 \mathrm{~h}$ to provide a SM: A ratio of 22.78 with $98 \%$ recovery of material. Adduct $\mathbf{5 0}$ was characterized as a pale yellow, crystalline solid, mp $<22^{\circ} \mathrm{C}$; IR (neat) $\mathrm{cm}^{-1}: 1707(\mathrm{C}=0$ ) ; ' $\mathrm{H}$ NMR $(400 \mathrm{MHz}): 1.01\left(\mathrm{~d}, 1 \mathrm{H}, J_{\mathrm{gem}}=11.8 \mathrm{~Hz}, \mathrm{H}-7 \alpha\right), 1.12\left(\mathrm{~s}, 3 \mathrm{H},-\mathrm{CH}_{3}\right)$, $1.91-2.03(\mathrm{~m}, 2 \mathrm{H}), 2.25-2.28(\mathrm{~m}, 2 \mathrm{H}), 2.42\left(\mathrm{dt}, 1 \mathrm{H}, J_{2 \beta, 3 \alpha}=\right.$ $\left.2.9 \mathrm{~Hz}, J_{2 \beta, 3 \alpha}=2.9 \mathrm{~Hz}, J_{g e m}=14.4 \mathrm{~Hz}, \mathrm{H}-2 \beta\right), 2.62(\mathrm{dd}, 1 \mathrm{H}$, $\left.J_{3 \alpha, 4 \alpha}=14.4 \mathrm{~Hz}, J_{\mathrm{gct} m}=19.4 \mathrm{~Hz}, \mathrm{H}-4 \beta\right), 2.86\left(\mathrm{dd}, 1 \mathrm{H}, J_{7 \beta, 8}=\right.$ $\left.5.1 \mathrm{~Hz}, J_{g e m}=11.8 \mathrm{~Hz}, \mathrm{H}-7 \beta\right), 4.82\left(\mathrm{dd}, 1 \mathrm{H}, J_{8,9}=1.6 \mathrm{~Hz}\right.$, $\left.J_{8,7 \beta}=5.1 \mathrm{~Hz}, \mathrm{H}-8\right), 6.14\left(\mathrm{~d}, 1 \mathrm{H}, J_{10.9}=5.7 \mathrm{~Hz}, \mathrm{H}-10\right), 6.49(\mathrm{dd}$, $\left.1 \mathrm{H}, J_{9.8}=1.6 \mathrm{~Hz}, J_{9,10}=5.7 \mathrm{~Hz}, \mathrm{H}-9\right) ;{ }^{13} \mathrm{C} \mathrm{NMR}(50 \mathrm{MHz}):$ $20.94,25.67,37.34$ and 38.28(t, C-2, C-3, C-4 and C-7), 22.44(q, $\left.-\mathrm{CH}_{3}\right), 54.18(\mathrm{~s}, \mathrm{C}-6), 78.03(\mathrm{~d}, \mathrm{C}-8), 91.70(\mathrm{~s}, \mathrm{C}-1), 135.13$ and 138.81(d, C-9 and C-10), 213.00(s, C-5); mass spectrum: 178(14, $\left.\mathrm{M}^{++}\right), 94\left(100, \mathrm{M}-\mathrm{CH}_{2}=\mathrm{C}(\mathrm{OH}) \mathrm{C}(\mathrm{Me})=\mathrm{CH}_{2}\right.$, retro IMDAFMcLafferty rearr.). Exact Mass calcd. for $\mathrm{C}_{11} \mathrm{H}_{14} \mathrm{O}_{2}$ : 178.0994; found: 178.0990

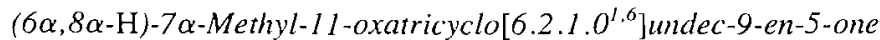
$(51)$

General procedure 5 was used to perform the Lewis acidmediated reactions of compound 3. Quantitative reaction: precursor 3 (107.6 mg, $0.604 \mathrm{mmol}$ ) was treated with $\mathrm{MeAlCl}_{2}$ $(664 \mu \mathrm{L}, 0.664 \mathrm{mmol})$ at $-78^{\circ} \mathrm{C}$ for $8 \mathrm{~h}$ to provide a $\mathrm{SM}$ : A ratio of $78: 22$ with quantitative recovery of material. Flash chromatography $(7: 1)$ provided starting material $(40.8 \mathrm{mg})$ and adduct $\mathbf{5 1}$ (11.2 mg). Catalytic reaction: enone 3 ( $121.6 \mathrm{mg}, 0.682 \mathrm{mmol})$ was treated with $\mathrm{MeAlCl}_{2}(68 \mu \mathrm{L}, 0.068 \mathrm{mmol})$ at $-65^{\circ} \mathrm{C}$ for $2 \mathrm{~h}$ to provide a SM : A ratio of $31: 69$ with $99 \%$ recovery of material. Flash chromatography $(9: 1)$ provided precursor $3(39.4 \mathrm{mg})$ and $51\left(77.8 \mathrm{mg}\right.$ ) as a clear, colourless oil; IR (neat) $\mathrm{cm}^{-1}: 1706(\mathrm{C}=\mathrm{O})$; ${ }^{\prime} \mathrm{H}$ NMR (400 MHz): $0.93\left(\mathrm{~d}, 3 \mathrm{H}, J=7.0 \mathrm{~Hz}, \mathrm{C}-7-\mathrm{CH}_{3}\right), 1.73(\mathrm{~d}$, $\left.1 \mathrm{H}, J_{6 \alpha, 7 \beta}=4.0 \mathrm{~Hz}, \mathrm{H}-6 \alpha\right), 1.82-2.01(\mathrm{~m}, 2 \mathrm{H}), 2.21(\mathrm{dt}, 1 \mathrm{H}$, $\left.J_{2 \alpha, 3 \alpha}=4.7 \mathrm{~Hz}, J_{2 \alpha, 3 \beta}=12.3 \mathrm{~Hz}, J_{\text {sem }}=12.7 \mathrm{~Hz}, \mathrm{H}-2 \alpha\right), 2.27-$ $2.38(\mathrm{~m}, 2 \mathrm{H}), 2.47\left(\mathrm{ddt}, 1 \mathrm{H}, J_{4 \beta .2 \beta}=1.6 \mathrm{~Hz}, J_{4 \beta, 3 \alpha}=3.7 \mathrm{~Hz}\right.$, $\left.J_{4 \beta, 3 \beta}=3.7 \mathrm{~Hz}, J_{\text {gem }}=14.4 \mathrm{~Hz}, \mathrm{H}-4 \beta\right), 2.79\left(\mathrm{ddq}, 1 \mathrm{H}, J_{7 \beta, 6 \alpha}=\right.$ $\left.4.0 \mathrm{~Hz}, J_{7 \beta, 8}=4.7 \mathrm{~Hz}, J=7.0 \mathrm{~Hz}, \mathrm{H}-7 \beta\right), 4.69\left(\mathrm{dd}, 1 \mathrm{H}, J_{8,9}=\right.$ $\left.1.6 \mathrm{~Hz}, J_{8,7 \beta}=4.7 \mathrm{~Hz}, \mathrm{H}-8\right), 6.24\left(\mathrm{~d}, 1 \mathrm{H}, J_{10.9}=5.7 \mathrm{~Hz}, \mathrm{H}-10\right)$, $6.37\left(\mathrm{dd}, 1 \mathrm{H}, J_{9.8}=1.6 \mathrm{~Hz}, J_{9.10}=5.7 \mathrm{~Hz}, \mathrm{H}-9\right) ;{ }^{13} \mathrm{C} \mathrm{NMR}$ $(50 \mathrm{MHz}): 17.23(\mathrm{q}), 21.45,28.50$ and $41.54(\mathrm{t}, \mathrm{C}-2, \mathrm{C}-3$, and $\mathrm{C}-4)$, $37.40(\mathrm{~d}, \mathrm{C}-7), 58.59$ (d, C-6), 81.80(d, C-8), 91.12(s, C-1), 135.80 and $138.32(\mathrm{~d}, \mathrm{C}-9$ and $\mathrm{C}-10), 209.76(\mathrm{~s}, \mathrm{C}-5)$; mass spectrum: $178\left(16, \mathrm{M}^{+}\right), 94\left(100, \mathrm{M}-\mathrm{CH}_{2}=\mathrm{C}(\mathrm{OH}) \mathrm{CMe}=\mathrm{CHMe}\right.$, retro IMDAF-McLafferty rearr.). Exact Mass calcd. for $\mathrm{C}_{11} \mathrm{H}_{14} \mathrm{O}_{2}$ : 178.0994; found: 178.1002 .

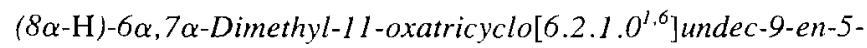
one (52)

General procedure 5 was used to perform the Lewis acidmediated reaction of compound 4. Quantitative reaction: enone 4 (38.1 $\mathrm{mg}, 0.198 \mathrm{mmol})$ was treated with $\mathrm{MeAlCl}_{2}(218 \mu \mathrm{L}$, $0.218 \mathrm{mmol}$ ) at $-65^{\circ} \mathrm{C}$ for $2 \mathrm{~h}$ to provide a SM: A ratio of $100: 0$. Catalytic reaction: compound $4(143.8 \mathrm{mg}, 0.747 \mathrm{mmol})$ was treated with $\mathrm{MeAlCl}_{2}(75 \mu \mathrm{L}, 0.075 \mathrm{mmol})$ at $-65^{\circ} \mathrm{C}$ for $2 \mathrm{~h}$ to provide a SM:A ratio of $95: 5$ (99\% recovery, $142.2 \mathrm{mg})$. Starting material $4(130.0 \mathrm{mg}$ ) could be separated from the product $\mathbf{5 2}$ (6.0 mg), a yellow oil, using flash chromatography (9:1) (no bp due to decomposition); 'H NMR (400 MHz): $0.78(\mathrm{~d}, 3 \mathrm{H}, J=$ $\left.7.4 \mathrm{~Hz}, \mathrm{C}-7-\mathrm{CH}_{3}\right), 0.93\left(\mathrm{~s}, 3 \mathrm{H}, \mathrm{C}-6-\mathrm{CH}_{3}\right), 1.88-2.00(\mathrm{~m}, 2 \mathrm{H})$, $2.20-2.26(\mathrm{~m}, 1 \mathrm{H}), 2.40\left(\mathrm{dt}, 1 \mathrm{H}, J_{4 \beta .3 \alpha}=3.0 \mathrm{~Hz}, J_{4 \beta, 3 \beta}=3.0 \mathrm{~Hz}\right.$, $\left.J_{g e m}=14.7 \mathrm{~Hz}, \mathrm{H}-4 \beta\right), 2.55-2.62(\mathrm{~m}, 1 \mathrm{H}), 2.64\left(\mathrm{dt}, 1 \mathrm{H}, J_{4 \alpha, 3 \alpha}=\right.$ $\left.7.2 \mathrm{~Hz}, J_{4 \alpha, 3 \beta}=14.7 \mathrm{~Hz}, J_{g e m}=14.7 \mathrm{~Hz}, \mathrm{H}-4 \alpha\right), 2.99(\mathrm{dq}, 1 \mathrm{H}$, $\left.J_{7 \beta, 8}=4.8 \mathrm{~Hz}, J_{7 \beta, 12}=7.4 \mathrm{~Hz}, \mathrm{H}-7 \beta\right), 4.71\left(\mathrm{dd}, J_{8,9}=1.7 \mathrm{~Hz}\right.$, $\left.J_{8,7 \beta}=4.8 \mathrm{~Hz}, \mathrm{H}-8\right), 6.22\left(\mathrm{~d}, 1 \mathrm{H}, J_{10.9}=5.8 \mathrm{~Hz}, \mathrm{H}-10\right), 6.45(\mathrm{dd}$, $\left.1 \mathrm{H}, J_{9,8}=1.7 \mathrm{~Hz}, J_{9,10}=5.8 \mathrm{~Hz}, \mathrm{H}-9\right) ;{ }^{13} \mathrm{C}$ NMR $(50 \mathrm{MHz})$ : 13.29 (q), 17.86 (q), 20.72, 26.18 and $38.20(\mathrm{t}, \mathrm{C}-2$ to $\mathrm{C}-4), 39.27$ (d, 
C-7), 60.35(s, C-6), 81.92(d, C-8), 92.68(s, C-1), 137.11(d, C-9 and $\mathrm{C}-10), 214.25(\mathrm{~s}, \mathrm{C}-5)$.

\section{$(6 \alpha-\mathrm{H})-8 \alpha-$ Methyl-11-oxatricyclo[6.2.1.0 $\left.0^{1,6}\right]$ undec-9-en-5-one} (53)

General procedure 5 was used to perform the Lewis acidmediated reaction of compound 5. Thus, enone $5(58.9 \mathrm{mg}$, $0.33 \mathrm{mmol})$ was treated with $\mathrm{MeAlCl}_{2}(0.36 \mathrm{~mL}, 0.36 \mathrm{mmol})$ at $-78^{\circ} \mathrm{C}$ for $1 \mathrm{~h}$ to provide adduct $53(58.9 \mathrm{mg}, 0.33 \mathrm{mmol})$ in $>99 \%$ yield as yellow oil (no bp due to decomposition on heating); IR (neat) $\mathrm{cm}^{-1}: 1710(\mathrm{C}=\mathrm{O}) ;{ }^{1} \mathrm{H}$ NMR $(400 \mathrm{MHz}): 1.58\left(\mathrm{~s}, 3 \mathrm{H},-\mathrm{CH}_{3}\right)$, $1.61\left(\mathrm{dd}, 1 \mathrm{H}, J_{6 \alpha, 7 \alpha}=8.2 \mathrm{~Hz}, J_{7 \alpha, 7 \beta}=11.8 \mathrm{~Hz}, \mathrm{H}-7 \alpha\right), 1.88-$ 2.04(m, $2 \mathrm{H}), 2.20\left(\mathrm{dd}, 1 \mathrm{H}, J_{7 \beta, 6 \alpha}=3.5 \mathrm{~Hz}, J_{g e m}=11.8 \mathrm{~Hz}, \mathrm{H}-7 \beta\right)$, $2.23\left(\mathrm{dt}, 1 \mathrm{H}, J_{2 \alpha, 3 \alpha}=5.2 \mathrm{~Hz}, J_{2 \alpha, 3 \beta}=12.4 \mathrm{~Hz}, J_{g e m}=12.4 \mathrm{~Hz}\right.$, $\mathrm{H}-2 \alpha), 2.39\left(\mathrm{dd}, 1 \mathrm{H}, J_{6 \alpha, 7 \beta}=3.5 \mathrm{~Hz}, J_{6 \alpha, 7 \alpha}=8.2 \mathrm{~Hz}, \mathrm{H}-6 \alpha\right), 2.35-$ 2.44(m, 2H), 2.52(dddt, $1 \mathrm{H}, J_{4 \beta, 2 \beta}=1.6 \mathrm{~Hz}, J_{4 \beta, 3 \alpha}=3.6 \mathrm{~Hz}, J_{4 \beta, 3 \beta}$ $\left.=3.6 \mathrm{~Hz}, J_{z c m}=14.1 \mathrm{~Hz}, \mathrm{H}-4 \beta\right), 6.15$ and $6.25(\mathrm{ABq}, 1 \mathrm{H}, J=$ 5.6- $\mathrm{Hz}, \mathrm{H}-9$ and $\mathrm{H}-10) ;{ }^{13} \mathrm{C}$ NMR $(50 \mathrm{MHz}): 18.71\left(\mathrm{q},-\mathrm{CH}_{3}\right)$, $21.68,28.54,35.42$, and $41.86(\mathrm{t}, \mathrm{C}-2, \mathrm{C}-3, \mathrm{C}-4$, and $\mathrm{C}-7)$, 53.77(d, C-6), 85.96 and $90.83(\mathrm{~s}, \mathrm{C}-1$ and $\mathrm{C}-8), 137.69$ and 141.18(d, C-9 and C-10), 209.70(s, C-5); mass spectrum (FI): 178. Exact mass calcd. for $\mathrm{C}_{11} \mathrm{H}_{14} \mathrm{O}_{2}$ : 178.0994; found: 178.0986 .

$6 \alpha, 8 \alpha$-Dimethyl-11-oxatricyclo[6.2.1.0 $\left.0^{I, 6}\right]$ undec-9-en-5-one (54)

General procedure 5 was used to perform the Lewis acidmediated reactions of compound 6. 1.1 Equivalents of $\mathrm{MeAlCl}_{2}$ reaction: precursor $6(80.5 \mathrm{mg}, 0.419 \mathrm{mmol})$ was treated with $\mathrm{MeAlCl}_{2}(461 \mu \mathrm{L}, 0.461 \mathrm{mmol})$ at $-78^{\circ} \mathrm{C}$ for $8 \mathrm{~h}$ to provide a SM: A ratio of 19:81 with quantitative recovery of material. Flash chromatography $(9: 1)$ provided starting material $(12.6 \mathrm{mg})$ and adduct $54(59.0 \mathrm{mg})$. Catalytic reaction: enone $6(37.4 \mathrm{mg}$, $0.192 \mathrm{mmol})$ was treated with $\mathrm{MeAlCl}_{2}(19 \mu \mathrm{L}, 0.019 \mathrm{mmol})$ at $-65^{\circ} \mathrm{C}$ for $2 \mathrm{~h}$ to provide a SM: A ratio of $<1:>99$ with $99 \%$ recovery of material. Flash chromatography $(9: 1)$ provided precursor $6(2.6 \mathrm{mg})$ and $54(34.6 \mathrm{mg})$ as a clear, colourless oil; IR (neat) $\mathrm{cm}^{-1}: 1708(\mathrm{C}=\mathrm{O}) ;{ }^{1} \mathrm{H}$ NMR $(400 \mathrm{MHz}): 1.08\left(\mathrm{~s}, 3 \mathrm{H}, \mathrm{C}-6-\mathrm{CH}_{3}\right)$, $1.10\left(\mathrm{~d}, 1 \mathrm{H}, J_{7 \alpha, 7 \beta}=5.5 \mathrm{~Hz}, \mathrm{H}-7 \alpha\right), 1.50\left(\mathrm{~s}, 3 \mathrm{H}, \mathrm{C}-8-\mathrm{CH}_{3}\right), 1.82-$ $2.04(\mathrm{~m}, 2 \mathrm{H}), 2.16-2.23(\mathrm{~m}, 2 \mathrm{H}), 2.38\left(\mathrm{dt}, 1 \mathrm{H}, J_{3 \beta .4 \beta}=3.2 \mathrm{~Hz}\right.$, $\left.J_{3 \alpha, 4 \beta}=3.2 \mathrm{~Hz}, J_{4 \alpha, 4 \beta}=14.4 \mathrm{~Hz}, \mathrm{H}-4 \beta\right), 2.51-2.67(\mathrm{~m}, 1 \mathrm{H})$, $2.55\left(\mathrm{~d}, 1 \mathrm{H}, J_{7 \alpha, 7 \beta}=11.8 \mathrm{~Hz}, \mathrm{H}-7 \beta\right), 6.13\left(\mathrm{~d}, 1 \mathrm{H}, J_{10.9}=5.6 \mathrm{~Hz}\right.$, $\mathrm{H}-10), 6.27\left(\mathrm{~d}, 1 \mathrm{H}, J_{9,10}=5.6 \mathrm{~Hz}, \mathrm{H}-9\right) ;{ }^{13} \mathrm{C}$ NMR (50 MHz): 18.87 and $22.24\left(\mathrm{q},-\mathrm{CH}_{3}\right.$ 's), 20.84; $25.86,38.22$, and $43.66(\mathrm{t}, \mathrm{C}-2, \mathrm{C}-3$, $\mathrm{C}-4$, and $\mathrm{C}-7$ ), 57.41 (s, C-6), 85.69 and 92.09 (s, C-1 and $\mathrm{C}-8$ ), 135.87 and 141.65 (d, C-9 and C-10), 213.33(s, C-5); mass spectrum: $192\left(5, \mathrm{M}^{+}\right), 108\left(100, \mathrm{M}-\mathrm{CH}_{2}=\mathrm{C}(\mathrm{OH}) \mathrm{C}(\mathrm{Me})=\mathrm{CH}_{2}\right.$, retro IMDAF-McLafferty rearr.). Exact Mass calcd. for $\mathrm{C}_{12} \mathrm{H}_{16} \mathrm{O}_{2}$ : 192.1150; found: 192.1141.

\section{$(6 \alpha-\mathrm{H})-7 \alpha, 8 \alpha$-Dimethyl-11-oxatricyclo $\left[6.2 .1 .0^{1.6}\right]$ undec-9-en-5- one (55)}

General procedure 5 was used to perform the Lewis acidmediated reaction of compound 7. Quantitative reaction: enone 7 $(63.7 \mathrm{mg}, 0.331 \mathrm{mmol})$ was treated with $\mathrm{MeAlCl}_{2}(364 \mu \mathrm{L}$, $0.364 \mathrm{mmol}$ ) at $-78^{\circ} \mathrm{C}$ for $8 \mathrm{~h}$ to provide a $\mathrm{SM}:$ A ratio of $82: 18$ with $98 \%$ recovery of material. Flash chromatography (7:1) provided enone $7(46.9 \mathrm{mg})$ and adduct $55(15.2 \mathrm{mg})$. Catalytic reaction: compound $7(51.4 \mathrm{mg}, 0.267 \mathrm{mmol})$ was treated with $\mathrm{MeAlCl}_{2}(27 \mu \mathrm{L}, 0.027 \mathrm{mmol})$ at $-65^{\circ} \mathrm{C}$ for $2 \mathrm{~h}$ to provide a SM:A ratio of $24: 76$ ( $92 \%$ recovery, $47.5 \mathrm{mg}$ ). Starting material 7 (10.7 mg) could be separated from the product $55(32.1 \mathrm{mg})$, a yellow solid, using flash chromatography $(9: 1), \mathrm{mp} 28-30^{\circ} \mathrm{C}$; IR $(\mathrm{KBr}) \mathrm{cm}^{-1}: 1709(\mathrm{C}=\mathrm{O}), 1136(\mathrm{C}-\mathrm{O})$; ${ }^{\mathrm{I}} \mathrm{H}$ NMR $(400 \mathrm{MHz})$ : $0.95\left(\mathrm{~d}, 3 \mathrm{H}, J=7.1 \mathrm{~Hz}, \mathrm{C}-7-\mathrm{CH}_{3}\right), 1.52\left(\mathrm{~s}, 3 \mathrm{H}, \mathrm{C}-8-\mathrm{CH}_{3}\right), 1.89(\mathrm{~d}$, $\left.1 \mathrm{H}, J_{6 \alpha .7 \beta}=4.3 \mathrm{~Hz}, \mathrm{H}-6 \alpha\right), 1.90-1.99(\mathrm{~m}, 2 \mathrm{H}), 2.17(\mathrm{ddd}, 1 \mathrm{H}$, $\left.J_{2 \alpha, 3 \alpha}=5.3 \mathrm{~Hz}, J_{2 \alpha, 3 \beta}=12.1 \mathrm{~Hz}, J_{g e m}=14.7 \mathrm{~Hz}, \mathrm{H}-2 \alpha\right), 2.29-$ $2.38(\mathrm{~m}, 2 \mathrm{H}), 2.46\left(\mathrm{dq}, 1 \mathrm{H}, J_{7 \beta, 6 \alpha}=4.1 \mathrm{~Hz}, J=7.0 \mathrm{~Hz}, \mathrm{H}-7 \beta\right)$, 2.5 (dddt, $1 \mathrm{H}, J_{4 \beta, 2 \beta}=1.6 \mathrm{~Hz}, J_{4 \beta, 3 \alpha}=3.7 \mathrm{~Hz}, J_{4 \beta, 3 \beta}=3.7 \mathrm{~Hz}$, $\left.J_{g e m}=14.3 \mathrm{~Hz}, \mathrm{H}-4 \beta\right), 6.20\left(\mathrm{~d}, 1 \mathrm{H}, J_{9,10}=5.6 \mathrm{~Hz}, \mathrm{H}-10\right), 6.26(\mathrm{~d}$, $\left.1 \mathrm{H}, J_{9,10}=5.6 \mathrm{~Hz}, \mathrm{H}-9\right) ;{ }^{13} \mathrm{C}$ NMR $(50 \mathrm{MHz}): 17.07(\mathrm{q}), 17.12(\mathrm{q})$, $21.38,28.62$, and $41.53(\mathrm{t}, \mathrm{C}-2, \mathrm{C}-3$, and C-4), 43.10(d, C-7), $61.51(\mathrm{~d}, \mathrm{C}-6), 88.63$ and $90.09(\mathrm{~s}, \mathrm{C}-1$ and $\mathrm{C}-8), 138.80$ and 138.87(d, C-9 and C-10), 210.14(s, C-5); mass spectrum: 192(13, $\left.\mathrm{M}^{*+}\right)$, $108\left(100, \mathrm{M}-\mathrm{CH}_{2}=\mathrm{C}(\mathrm{OH}) \mathrm{CH}=\mathrm{CHMe}\right.$, retro IMDAFMcLafferty rearr.). Exact Mass calcd. for $\mathrm{C}_{12} \mathrm{H}_{16} \mathrm{O}_{2}$ : 192.1151; found: 192.1132 .

$(6 \alpha, 8 \alpha-\mathrm{H})-2,2-D i m e t h y l-11$-oxatricyclo[6.2.1.0.6 $]$ undec-9-en-5one (56)

The IMDAF reaction of compound 9 ( $52.9 \mathrm{mg}, 0.275 \mathrm{mmol})$ was performed according to general procedure 4 to provide adduct $\mathbf{5 6}$ ( $46.5 \mathrm{mg}, 0.242 \mathrm{mmol}$ ) after $12 \mathrm{~h}$ in $88 \%$ yield as a white solid, $\mathrm{mp} 57-60^{\circ} \mathrm{C}$; IR $(\mathrm{KBr}) \mathrm{cm}^{-1}: 1705(\mathrm{C}=\mathrm{O}), 1663(\mathrm{C}=\mathrm{C}), 1390$, $1369(\mathrm{gem}-\mathrm{Me}) ;{ }^{1} \mathrm{H}$ NMR (400 MHz): 1.08 and $1.36(\mathrm{~s}, 6 \mathrm{H}, 2 \times$ $\left.\mathrm{CH}_{3}\right), 1.55$ (dd, $1 \mathrm{H}, J_{7 \alpha .6 \alpha}=8.5 \mathrm{~Hz}, J_{\text {gem }}=11.8 \mathrm{~Hz}, \mathrm{H}-7 \alpha$ ), 1.61 (ddd, $1 \mathrm{H}, J_{3 \alpha, 4 \beta}=2.7 \mathrm{~Hz}, J_{3 \alpha, 4 \alpha}=5.8 \mathrm{~Hz}, J_{\text {dem }}=13.9 \mathrm{~Hz}$, $\mathrm{H}-3 \alpha), 1.99\left(\mathrm{dt}, 1 \mathrm{H}, J_{3 \beta .4 \beta}=4.3 \mathrm{~Hz}, J_{3 \beta, 4 \alpha}=13.9 \mathrm{~Hz}, J_{g e m}=\right.$ $13.9 \mathrm{~Hz}, \mathrm{H}-3 \beta), 2.27$ (dd, $1 \mathrm{H}, J_{6 \alpha, 7 \beta}=3.2 \mathrm{~Hz}, J_{6 \alpha, 7 \alpha}=8.5 \mathrm{~Hz}$, $\mathrm{H}-6 \alpha$ ), 2.37-2.42(overlapping $\mathrm{m}, 2 \mathrm{H}, \mathrm{H}-4 \beta$ and $\mathrm{H}-7 \beta$ ), 2.58 (dt, $\left.1 \mathrm{H}, J_{4 \alpha, 3 \alpha}=5.8 \mathrm{~Hz}, J_{4 \alpha, 3 \beta}=15.1 \mathrm{~Hz}, J_{4 \alpha, 4 \beta}=15.1 \mathrm{~Hz}, \mathrm{H}-4 \alpha\right)$, $4.88\left(\mathrm{dd}, 1 \mathrm{H}, J_{8.9}=1.5 \mathrm{~Hz}, J_{8.7 \mathrm{~B}}=4.9 \mathrm{~Hz}, \mathrm{H}-8\right), 6.24(\mathrm{~d}, 1 \mathrm{H}$, $\left.J_{10,9}=5.8 \mathrm{~Hz}, \mathrm{H}-10\right), 6.43\left(\mathrm{dd}, \mathrm{iH}, J_{9,8}=1.5 \mathrm{~Hz}, J_{9,10}=5.8 \mathrm{~Hz}\right.$, $\mathrm{H}-9) ;{ }^{13} \mathrm{C}$ NMR (50 MHz): 24.24 and $25.96\left(\mathrm{q},-\mathrm{CH}_{3}\right), 30.98,34.88$, and $37.84(\mathrm{t}, \mathrm{C}-3, \mathrm{C}-4$, and $\mathrm{C}-7), 31.98(\mathrm{~s}, \mathrm{C}-2), 47.90(\mathrm{~d}, \mathrm{C}-6)$, 77.90(d, C-8), 95.90(s, C-1), 133.47, 138.83(d, C-9 and C-10), 210.20(s, C-5); mass spectrum: 192(48, $\left.\mathrm{M}^{+}\right), 109(100, \mathrm{M}-$ $\left.\left(\mathrm{CH}_{2}\right)_{2} \mathrm{C}(\mathrm{O}) \mathrm{CH}=\mathrm{CH}_{2}\right)$. Exact Mass calcd. for $\mathrm{C}_{12} \mathrm{H}_{16} \mathrm{O}_{2}: 192.1150$; found: 192,1148 .

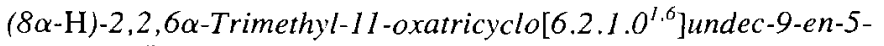
one (57)

The IMDAF reaction of compound $\mathbf{1 0}(221.2 \mathrm{mg}, 1.07 \mathrm{mmol})$ was performed according to general procedure 4 to provide after $96 \mathrm{~h}$ precursor $10(186.4 \mathrm{mg}, 0.903 \mathrm{mmol})$ and adduct 57 (19.8 $\mathrm{mg}, 0.096 \mathrm{mmol}$ ) after purification by flash chromatography $(9: 1)$ in $93 \%$ total recovery.

General Procedure 5 was used to perform the Lewis acidmediated reactions of compound 10. Thus, enone $\mathbf{1 0}(55.3 \mathrm{mg}$, $0.268 \mathrm{mmol}$ ) was treated with quantitative $\mathrm{MeAlCl}_{2}(295 \mu \mathrm{L}$, $0.295 \mathrm{mmol}$ ) at $-78^{\circ} \mathrm{C}$ for $8 \mathrm{~h}$ to provide a SM: A ratio of $68: 32$ with a quantitative recovery of material. Enone $10(161.9 \mathrm{mg}$, $0.785 \mathrm{mmol}$ ) was treated with catalytic $\mathrm{MeAlCl}_{2}(79 \mu \mathrm{L}$, $0.079 \mathrm{mmol}$ ) (according to general procedure 5) at $-65^{\circ} \mathrm{C}$ for $2 \mathrm{~h}$ to provide a SM: A ratio of 40:60 with quantitative recovery. Flash chromatography $(9: 1)$ provided enone $10(64.5 \mathrm{mg})$ and adduct 57 $(92.3 \mathrm{mg})$ in $97 \%$ recovered yield. Adduct 57 was characterized as a white solid, $\mathrm{mp} 44-55^{\circ} \mathrm{C}$; IR $(\mathrm{KBr}) \mathrm{cm}^{-1}$ : $3078,3021(\mathrm{C}-\mathrm{H})$, 2982-2858(C-H), 1699(C=O), 1389, $1369(\mathrm{gem}-\mathrm{Me}) ;{ }^{1} \mathrm{H}$ NMR $(200 \mathrm{MHz}): 0.99(\mathrm{~d}, 1 \mathrm{H}, J=11.7 \mathrm{~Hz}, \mathrm{H}-7 \alpha), 1.03\left(\mathrm{~s}, \mathrm{C}-6-\mathrm{CH}_{3}\right)$, 1.16 and $1.39\left(\mathrm{~s}, 3 \mathrm{H}\right.$ each, $\left.2 \times \mathrm{CH}_{3}\right), 1.61$ (ddd, $1 \mathrm{H}, J_{3 \alpha, 4 \beta}=$ $\left.2.8 \mathrm{~Hz}, J_{3 \alpha, 4 \alpha}=5.8 \mathrm{~Hz}, J_{3 \alpha, 3 \beta}=13.4 \mathrm{~Hz}, \mathrm{H}-3 \alpha\right), 2.01(\mathrm{dt}, 1 \mathrm{H}$, $\left.J_{3 \beta, 4 \beta}=4.1 \mathrm{~Hz}, J_{3 \beta, 4 \alpha}=14.3 \mathrm{~Hz}, J_{3 \beta, 3 \alpha}=13.6 \mathrm{~Hz}, \mathrm{H}-3 \beta\right)$, 2.35 (ddd, $1 \mathrm{H}, J_{4 \beta, 3 \alpha}=2.8 \mathrm{~Hz}, J_{4 \beta, 3 \beta}=4.0 \mathrm{~Hz}, J_{4 \beta, 4 \alpha}=15.3 \mathrm{~Hz}$, $\mathrm{H}-4 \beta), 2.82\left(\mathrm{dt}, 1 \mathrm{H}, J_{4 \beta, 3 \beta}=5.8 \mathrm{~Hz}, J_{g e m}=14.5 \mathrm{~Hz}, \mathrm{H}-4 \alpha\right)$, $2.85\left(\mathrm{dd}, 1 \mathrm{H}, J_{7 \beta, 8}=3.8 \mathrm{~Hz}, J_{7 \beta, 7 \alpha}=11.8 \mathrm{~Hz}, \mathrm{H}-7 \beta\right), 4.77(\mathrm{dd}, 1 \mathrm{H}$, $\left.J_{8,9}=1.6 \mathrm{~Hz}, J_{8,7 \beta}=5.2 \mathrm{~Hz}, \mathrm{H}-8\right), 6.33\left(\mathrm{~d}, 1 \mathrm{H}, J_{10,9}=5.9 \mathrm{~Hz}\right.$, $\mathrm{H}-10), 6.48\left(\mathrm{dd}, 1 \mathrm{H}, J_{98}=1.7 \mathrm{~Hz}, J_{9.10}=5.9 \mathrm{~Hz}, \mathrm{H}-9\right) ;{ }^{13} \mathrm{C} \mathrm{NMR}$ $(50 \mathrm{MHz}): 23.85(\mathrm{q}), 24.44(\mathrm{q}), 27.48(\mathrm{q}), 32.70(\mathrm{~s}, \mathrm{C}-2), 35.10$, 35.29 , and 39.78(t, C-3, C-4, and C-7), 54.18(s, C-6), 77.39(d, $\mathrm{C}-8), 97.10(\mathrm{~s}, \mathrm{C}-1), 132.15$ and $138.70(\mathrm{~d}, \mathrm{C}-9$ and $\mathrm{C}-10)$, 213.94(s, C-5); mass spectrum: 206(10, $\left.\mathrm{M}^{*+}\right), 109(100, \mathrm{M}-$ $\left.\left(\mathrm{CH}_{2}\right)_{2} \mathrm{C}(\mathrm{O}) \mathrm{CMe}=\mathrm{CH}_{2}\right)$. Exact Mass calcd. for $\mathrm{C}_{13} \mathrm{H}_{18} \mathrm{O}_{2}$ : 206.1307; found: 206.1290 .

$(6 \alpha, 8 \alpha-\mathrm{H})-2,2,7 \alpha$-Trimethyl-11-oxatricyclo $\left[6.2 .1 .0^{1,6}\right]$ undec-9en-5-one (58)

The IMDAF reaction of compound $11(54.9 \mathrm{mg}, 0.266 \mathrm{mmol})$ was performed according to general procedure 4 to provide a SM:A ratio of 87:13 with quantitative recovery of material after $192 \mathrm{~h}$. General procedure 5 was used to perform the Lewis acid-mediated reactions of compound 11. Thus, enone 11 (104.1 mg, $0.504 \mathrm{mmol}$ ) was treated with quantitative $\mathrm{MeAlCl}_{2}(555 \mu \mathrm{L}$, $0.555 \mathrm{mmol}$ ) at $-78^{\circ} \mathrm{C}$ for $8 \mathrm{~h}$ to provide a SM:A ratio of $73: 27$ 
and enone $11(60.5 \mathrm{mg})$ and adduct $58(24.7 \mathrm{mg})$ with $82 \%$ recovery after separation by flash chromatography (9:1). Enone 11 (124.7 $\mathrm{mg}, 0.604 \mathrm{mmol}$ ) was treated with catalytic $\mathrm{MeAlCl}_{2}$ $(60 \mu \mathrm{L}, 0.060 \mathrm{mmol})$ at $-65^{\circ} \mathrm{C}$ for $2 \mathrm{~h}$ according to general procedure 5 to provide a SM: A ratio of $27: 73$ with a quantitative recovery of material. Adduct $\mathbf{5 8}$ was characterized as a solid, mp 35$37^{\circ} \mathrm{C}$; IR $(\mathrm{KBr}) \mathrm{cm}^{-1}: 3012(\mathrm{CH}), 2968-2871(\mathrm{CH}), 1698(\mathrm{C}=\mathrm{O})$, 1389, $1368(\mathrm{gem}-\mathrm{Me})$; ' $\mathrm{H}$ NMR $(200 \mathrm{MHz}): 0.98\left(\mathrm{~d}, J_{7 \beta .14}=\right.$ $\left.7.0 \mathrm{~Hz}, \mathrm{C}-7-\mathrm{CH}_{3}\right), 1.07$ and $1.31(\mathrm{~s}, 3 \mathrm{H}$ each), $1.59(\mathrm{ddd}, 1 \mathrm{H}$, $\left.J_{3 \alpha, 4 \beta}=3.1 \mathrm{~Hz}, J_{3 \alpha, 4 \alpha}=5.7 \mathrm{~Hz}, J_{3 \alpha, 3 \beta}=13.4 \mathrm{~Hz}, \mathrm{H}-3 \alpha\right), 1.77(\mathrm{~d}$, $\left.1 \mathrm{H}, J_{6 \alpha, 7 \beta}=4.0 \mathrm{~Hz}, \mathrm{H}-6 \alpha\right), 2.01\left(\mathrm{dt}, 1 \mathrm{H}, J_{3 \beta, 4 \beta}=5.2 \mathrm{~Hz}, J_{3 \beta, 4 \alpha}=\right.$ $13.4 \mathrm{~Hz}, J_{3 \alpha, 3 \beta}=13.4 \mathrm{~Hz}, \mathrm{H}-3 \beta$ ), 2.40 (ddd, $1 \mathrm{H}, J_{3 \alpha .4 \beta}=3.1 \mathrm{~Hz}$, $J_{3 \beta, 4 \beta}=5.2 \mathrm{~Hz}, J_{4 \alpha, 4 \beta}=16.3 \mathrm{~Hz}, \mathrm{H}-4 \beta$ ), 2.54 (ddd, $1 \mathrm{H}, J_{3 \alpha, 4 \alpha}=$ $\left.6.6 \mathrm{~Hz}, J_{3 \beta, 4 \alpha}=13.4 \mathrm{~Hz}, J_{4 \beta, 4 \alpha}=16.2 \mathrm{~Hz}, \mathrm{H}-4 \alpha\right), 2.74(\mathrm{ddq}, 1 \mathrm{H}$, $\left.J_{7 \beta, 6 \alpha}=4.2 \mathrm{~Hz}, J_{7 \beta, 8}=4.8 \mathrm{~Hz}, J_{7 \beta, 14}=7.0 \mathrm{~Hz}, \mathrm{H}-7 \beta\right), 4.73(\mathrm{~d}$, $\left.1 \mathrm{H}, J_{7 \beta, 8}=4.6 \mathrm{~Hz}, \mathrm{H}-8\right), 6.40(\mathrm{ABq}, 2 \mathrm{H}, \mathrm{H}-9$ and $\mathrm{H}-10) ;{ }^{13} \mathrm{C} \mathrm{NMR}$ (50 MHz): 17.51(q), 24.23(q), 25.78(q), 31.91(s, C-2), 34.47 and $37.55(\mathrm{t}, \mathrm{C}-3$ and $\mathrm{C}-4), 39.72(\mathrm{~d}, \mathrm{C}-7), 56.28(\mathrm{~d}, \mathrm{C}-6), 81.57(\mathrm{~d}, \mathrm{C}-8)$, 96.47(s, C-1), 134.95, 136.32(d, C-9 and C-10), 211.29(s, C-5); mass spectrum: 206(20, $\left.\mathrm{M}^{*+}\right), 109\left(100, \mathrm{M}-\left(\mathrm{CH}_{2}\right)_{2} \mathrm{C}(\mathrm{O}) \mathrm{CMe}=\right.$ $\mathrm{CH}_{2}$ ). Exact Mass calcd. for $\mathrm{C}_{13} \mathrm{H}_{18} \mathrm{O}_{2}: 206.1307$; found: 206.1305 .

$(6 \alpha-\mathrm{H})-2,2,8 \alpha$-Trimethyl-11-oxatricyclo $\left[6.2 .1 .0^{1.6}\right]$ undec-9-en-5one $(60)$

The IMDAF reaction of compound $13(62.1 \mathrm{mg}, 0.301 \mathrm{mmol})$ occurred at $0^{\circ} \mathrm{C}$ as a neat oil to provide adduct $60(62.1 \mathrm{mg}$, $0.301 \mathrm{mmol}$ ) as a white, crystalline solid, $\mathrm{mp} 52-54^{\circ} \mathrm{C}$; IR $(\mathrm{KBr})$ $\mathrm{cm}^{-1}: 1707(\mathrm{C}=\mathrm{O}) ;{ }^{1} \mathrm{H}$ NMR $(300 \mathrm{MHz}): 1.08$ and $1.33(\mathrm{~s}, 3 \mathrm{H}$ each), $1.55\left(\mathrm{~s}, 3 \mathrm{H} \mathrm{C}-8-\mathrm{CH}_{3}\right), 1.60\left(\mathrm{ddd}, 1 \mathrm{H}, J_{3 \alpha, 4 \beta}=2.7 \mathrm{~Hz}, J_{3 \alpha, 4 \alpha}\right.$ to $\left.5.8 \mathrm{~Hz}, J_{3 \alpha, 3 \beta}=13.9 \mathrm{~Hz}, \mathrm{H}-3 \alpha\right), 1.68\left(\mathrm{dd}, 1 \mathrm{H}, J_{7 \alpha, 6 \alpha}=8.4 \mathrm{~Hz}\right.$, $\left.J_{7 \alpha, 7 \beta}=11.8 \mathrm{~Hz}, \mathrm{H}-7 \alpha\right), 2.01\left(\mathrm{dt}, 1 \mathrm{H}, J_{3 \beta, 4 \beta}=4.3 \mathrm{~Hz}, J_{3 \beta, 4 \alpha}=\right.$ $\left.13.9 \mathrm{~Hz}, J_{3 \beta, 3 \alpha}=13.9 \mathrm{~Hz}, \mathrm{H}-3 \beta\right), 2.12\left(\mathrm{dd}, 1 \mathrm{H}, J_{7 \beta, 6 \alpha}=3.3 \mathrm{~Hz}\right.$, $\left.J_{7 \beta, 7 \alpha}=11.8 \mathrm{~Hz}, \mathrm{H}-7 \beta\right), 2.38\left(\mathrm{dd}, 1 \mathrm{H}, J_{6 \alpha, 7 \beta}=3.3 \mathrm{~Hz}, J_{6 \alpha, 7 \alpha}=\right.$ $8.4 \mathrm{~Hz}, \mathrm{H}-6 \alpha$ ), 2.40 (ddd, $1 \mathrm{H}, J_{4 \beta, 3 \alpha}=2.8 \mathrm{~Hz}, J_{4 \beta, 3 \beta}=4.3 \mathrm{~Hz}$, $\left.J_{4 \beta, 4 \alpha}=13.2 \mathrm{~Hz}, \mathrm{H}-4 \beta\right), 2.56\left(\mathrm{dt}, 1 \mathrm{H}, J_{4 \alpha, 3 \alpha}=5.8 \mathrm{~Hz}, J_{4 \alpha, 4 \beta}=\right.$ $\left.13.2 \mathrm{~Hz}, J_{4 \alpha, 3 \beta}=13.9 \mathrm{~Hz}, \mathrm{H}-4 \alpha\right), 6.25(\mathrm{ABq}, 2 \mathrm{H}, \mathrm{H}-9$ and $\mathrm{H}-10)$; ${ }^{13} \mathrm{C}$ NMR (75 MHz): 18.77(q), 24.08(q), 26.05(q), 32.10(s, C-2), $34.88,37.40$, and $37.89(\mathrm{t}, \mathrm{C}-3, \mathrm{C}-4$, and C-7), 51.24(d, C-6), 85.63 and 96.09 (s, C-1 and C-8), 134.16 and 141.77 (d, C-9 and C-10), 210.83(s, C-5); mass spectrum: 206(43, $\left.\mathrm{M}^{\circ+}\right), 191(13$, $\mathrm{M}-\mathrm{Me}), 123\left(100, \mathrm{M}-\left(\mathrm{CH}_{2}\right)_{2} \mathrm{C}(\mathrm{O}) \mathrm{CH}=\mathrm{CH}_{2}\right)$. Exact Mass calcd. for $\mathrm{C}_{13} \mathrm{H}_{18} \mathrm{O}_{2}$ : 206.1307; found: 206.1297.

\section{$2,2,6 \alpha, 8 \alpha$-Tetramethyl-11-oxatricyclo $\left[6.2,1.0^{1.6}\right]$ undec-9-en-5- one $(61)$}

The IMDAF reaction of compound $14(58.4 \mathrm{mg}, 0.265 \mathrm{mmol})$ was performed according to general procedure 4 to provide a SM:A ratio of $88: 12$ in $82 \%$ recovery of material after $336 \mathrm{~h}$. Starting material $(40.9 \mathrm{mg})$ and adduct $(6.8 \mathrm{mg})$ were isolated by flash chromatography $(9: 1)$. General procedure 5 was used to perform the Lewis acid-mediated reactions of compound 14. Thus, enone 14 (48.8 mg, $0.222 \mathrm{mmol}$ ) was treated with quantitative $\mathrm{MeAlCl}_{2}$ $\left(244 \mu \mathrm{L}, 0.244 \mathrm{mmol}\right.$ ) at $-78^{\circ} \mathrm{C}$ for $8 \mathrm{~h}$ to provide a SM: A ratio of $78: 22$ with quantitative recovery of material. Enone 14 $(30.1 \mathrm{mg})$ and adduct $(9.0 \mathrm{mg})$ were isolated by flash chromatography $(9: 1)$. Enone $14(32.7 \mathrm{mg}, 0.148 \mathrm{mmol})$ was treated with catalytic $\mathrm{MeAlCl}_{2}(15 \mu \mathrm{L}, 0.015 \mathrm{mmol}$ ) (according to general procedure 5) at $-65^{\circ} \mathrm{C}$ for $2 \mathrm{~h}$ to provide a SM:A ratio of $23: 77$ with quantitative recovery. Adduct 61 was characterized as a solid; IR $(\mathrm{KBr}) \mathrm{cm}^{-1}:$ 2986-2873(C-H), 1708(C=O), 1384, 1371 ( $\mathrm{gem}-$ $\mathrm{Me}) ;{ }^{1} \mathrm{H}$ NMR $(300 \mathrm{MHz}): 1.01\left(\mathrm{~s}, 3 \mathrm{H}, \mathrm{C}-6-\mathrm{CH}_{3}\right), 1.09(\mathrm{~d}, 1 \mathrm{H}$, $\left.J_{7 \alpha, 7 \beta}=11.7 \mathrm{~Hz}, \mathrm{H}-7 \alpha\right), 1.12$ and $1.33(\mathrm{~s}, 3 \mathrm{H}$ each), $1.46(\mathrm{~s}, 3 \mathrm{H}$, C-8- $\mathrm{CH}_{3}$ ), 1.56 (ddd, $1 \mathrm{H}, J_{3 \alpha, 4 \beta}=2.7 \mathrm{~Hz}, J_{3 \alpha, 4 \alpha}=5.8 \mathrm{~Hz}$, $\left.J_{3 \alpha, 3 \beta}=13.6 \mathrm{~Hz}, \mathrm{H}-3 \alpha\right), 1.99\left(\mathrm{dt}, 1 \mathrm{H}, J_{3 \beta .4 \beta}=4.1 \mathrm{~Hz}, J_{3 \beta, 3 \alpha}=\right.$ $13.6 \mathrm{~Hz}, J_{3 \beta, 4 \alpha}=14.4 \mathrm{~Hz}, \mathrm{H}-3 \beta$ ), 2.32 (ddd, $1 \mathrm{H}, J_{4 \beta, 3 \alpha}=2.7 \mathrm{~Hz}$, $\left.J_{4 \beta, 3 \beta}=4.1 \mathrm{~Hz}, J_{4 \beta, 4 \alpha}=15.3 \mathrm{~Hz}, \mathrm{H}-4 \beta\right), 2.53(\mathrm{~d}, 1 \mathrm{H}, J=$ $11.7 \mathrm{~Hz}, \mathrm{H}-7 \beta$ ), 2.77 (ddd, $1 \mathrm{H}, J_{4 \alpha, 3 \alpha}=5.8 \mathrm{~Hz}, J_{4 \alpha, 3 \beta}=14.4 \mathrm{~Hz}$, $\left.J_{4 \alpha, 4 \beta}=15.3 \mathrm{~Hz}, \mathrm{H}-4 \alpha\right), 6.25\left(\mathrm{~d}, 1 \mathrm{H}, J_{10,9}=5.7 \mathrm{~Hz}, \mathrm{H}-10\right), 6.30(\mathrm{~d}$, $\left.1 \mathrm{H}, J_{9,10}=5.7 \mathrm{~Hz}, \mathrm{H}-9\right) ;{ }^{13} \mathrm{C}$ NMR (75 MHz): 18.93(q), 23.75(q), 24.27(q), 27.51(q), 32.78(s, C-2), 35.00 and $35.31(\mathrm{t}, \mathrm{C}-3$ and $\mathrm{C}-7)$, 46.21(t, C-4), 57.37(s, C-6), 84.76 and 97.29(s, C-1 and C-8), 132.76 and 141.69 (d, C-9 and C-10), 214.39(s, C-5); mass spectrum: $220\left(18, \mathrm{M}^{+}\right), 123\left(100, \mathrm{M}-\left(\mathrm{CH}_{2}\right)_{2} \mathrm{C}(\mathrm{O}) \mathrm{CMe}=\mathrm{CH}_{2}\right)$. Exact Mass calcd. for $\mathrm{C}_{14} \mathrm{H}_{20} \mathrm{O}_{2}: 220,1464$; found: 220.1462.

$(6 \alpha-\mathrm{H})-2,2,7 \alpha, 8 \alpha$-Tetramethyl-11-oxatricyclo $\left[6.2 .1 .0^{1,6}\right]$ undec-

9-en-5-one (62)

General procedure 5 was used to perform the Lewis acidmediated reactions of compound 15 . Thus, enone 15 (14.6 mg, $0.066 \mathrm{mmol}$ ) was treated with catalytic $\mathrm{MeAlCl}_{2}$ (6.6 $\mu \mathrm{L}$, $0.066 \mathrm{mmol}$ ) at $-65^{\circ} \mathrm{C}$ for $2 \mathrm{~h}$ to provide a $15: 62$ ratio of $69: 31$ with $72 \%$ recovery; IR $(\mathrm{KBr}) \mathrm{cm}^{-1}: 2960-2868(\mathrm{C}-\mathrm{H}), 1706(\mathrm{C}=\mathrm{O})$, $1384,1368(\mathrm{gem}-\mathrm{Me})$; 'H NMR (400 MHz): $0.97\left(\mathrm{~d}, 3 \mathrm{H}, J_{14,7 \beta}=\right.$ $7.0 \mathrm{~Hz}, \mathrm{H}-14), 1.08$ and $1.28(\mathrm{~s}, 3 \mathrm{H}$ each $), 1.50\left(\mathrm{~s}, 3 \mathrm{H}, \mathrm{C}-8-\mathrm{CH}_{3}\right)$, $1.56\left(\mathrm{ddd}, 1 \mathrm{H}, J_{3 \alpha, 4 \beta}=2.8 \mathrm{~Hz}, J_{3 \alpha, 4 \alpha}=6.0 \mathrm{~Hz}, J_{3 \alpha, 3 \beta}=13.5 \mathrm{~Hz}\right.$, $\mathrm{H}-3 \alpha), 1.88\left(\mathrm{~d}, 1 \mathrm{H}, J_{6 \alpha, 7 \beta}=4.1 \mathrm{~Hz}, \mathrm{H}-6 \alpha\right), 2.01\left(\mathrm{dt}, 1 \mathrm{H}, J_{3 \beta, 4 \beta}=\right.$ $\left.4.8 \mathrm{~Hz}, J_{3 \beta .4 \alpha}=13.6 \mathrm{~Hz}, J_{3 \beta, 3 \alpha}=13.6 \mathrm{~Hz}, \mathrm{H}-3 \beta\right), 2.35(\mathrm{dq}, 1 \mathrm{H}$, $\left.J_{7 \beta, 6 \alpha}=4.1 \mathrm{~Hz}, J_{7 \beta, 14}=7.0 \mathrm{~Hz}, \mathrm{H}-7 \beta\right), 2.40\left(\mathrm{ddd}, 1 \mathrm{H}, J_{4 \beta, 3 \alpha}=\right.$ $\left.2.8 \mathrm{~Hz}, J_{4 \beta, 3 \beta}=4.8 \mathrm{~Hz}, J_{4 \beta, 4 \alpha}=16.2 \mathrm{~Hz}, \mathrm{H}-4 \beta\right), 2.51$ (ddd, $1 \mathrm{H}$, $\left.J_{4 \alpha, 3 \alpha}=6.0 \mathrm{~Hz}, J_{4 \alpha, 3 \beta}=13.6 \mathrm{~Hz}, J_{4 \alpha, 4 \beta}=16.2 \mathrm{~Hz}, \mathrm{H}-4 \alpha\right), 6.20(\mathrm{~d}$, $\left.1 \mathrm{H}, J_{10,9}=5.7 \mathrm{~Hz}, \mathrm{H}-10\right), 6.37\left(\mathrm{~d}, 1 \mathrm{H}, J_{9,10}=5.7 \mathrm{~Hz}, \mathrm{H}-9\right) ;{ }^{13} \mathrm{C}$ NMR (50 MHz): 17.20(q), 17.40(q), 24.06(q), 25.86(q), 31.94(s, C-2), 34.40 and $37.55(\mathrm{t}, \mathrm{C}-3$ and $\mathrm{C} 4), 45.65(\mathrm{~d}, \mathrm{C}-7), 59.21(\mathrm{~d}$, C-6), 88.25 and 95.38(s, C-1 and C-8), 135.41 and 139.34(d, C-9 and $\mathrm{C}-10), 211.59(\mathrm{~s}, \mathrm{C}-5)$; mass spectrum: $220\left(8, \mathrm{M}^{*+}\right), 123(100$, $\left.\mathrm{M}-\left(\mathrm{CH}_{2}\right)_{2} \mathrm{C}(\mathrm{O}) \mathrm{CH}=\mathrm{CHMe}\right)$. Exact Mass calcd. for $\mathrm{C}_{14} \mathrm{H}_{20} \mathrm{O}_{2}$ : 220.1464; found: 220.1465

\section{$(7 \beta, 9 \alpha-\mathrm{H})$-12-Oxatricyclo[7.2.1.0 $\left.0^{\prime .7}\right]$ dodec-10-en-6-one (63)}

General procedure 5 was used to perform the Lewis acidmediated reaction of compound 8 . Thus, enone $8(63.5 \mathrm{mg}$, $0.356 \mathrm{mmol})$ was treated with $\mathrm{MeAlCl}_{2}(36 \mu \mathrm{L}, 0.036 \mathrm{mmol})$ at $-78^{\circ} \mathrm{C}$ for $2 \mathrm{~h}$ to provide a SM: A ratio of $8: 92$ with $98 \%$ recovery of material. Some retro-IMDAF reaction occurred upon attempted purification by flash chromatography $(9: 1)$. Adduct 63 was characterized as a white crystalline solid; 'H NMR $(200 \mathrm{MHz})$ : $1.38-1.67(\mathrm{~m}, 2 \mathrm{H}), 1.75-2.30$ (overlapping $\mathrm{m}, 6 \mathrm{H}), 2.38$ 2.70 (overlapping $\mathrm{m}, 2 \mathrm{H}), 3.20(\mathrm{dd}, 1 \mathrm{H}), 4.90(\mathrm{dd}, 1 \mathrm{H}, \mathrm{H}-9)$, $6.02\left(\mathrm{~d}, 1 \mathrm{H}, J_{11,10}=6.2 \mathrm{~Hz}, \mathrm{H}-11\right), 6.41\left(\mathrm{dd}, 1 \mathrm{H}, J_{10,9}=1.2 \mathrm{~Hz}\right.$, $\left.J_{10,11}=6.2 \mathrm{~Hz}, \mathrm{H}-10\right)$.

\section{2,2-Dimethyl-7-(trimethysilyl)-11-oxatricyclo[6.2.1.0 $\left.0^{1.6}\right]$ undec- 6,9-dien-5-one (65)}

General procedure 5 was used for the Lewis acid-mediated IMDAF reaction of compound 16. Thus, enone $16(100.9 \mathrm{mg}$, $0.384 \mathrm{mmol})$ was treated with $\mathrm{MeAlCl}_{2}(423 \mu \mathrm{L}, 0.423 \mathrm{mmol})$ at $-50^{\circ} \mathrm{C}$ for $2.5 \mathrm{~h}$ to provide a $\mathrm{SM}: \mathrm{A}$ ratio of 12.88 . Adduct 65 $(76.4 \mathrm{mg}, 0.482 \mathrm{mmol}$ ) was obtained in $89 \%$ yield (based on recovered starting material) as a golden crystalline solid, after purification by flash chromatography; mp $85-94^{\circ} \mathrm{C}$; IR $(\mathrm{Kbr}) \mathrm{cm}^{-1}$ : $1660(\mathrm{C}=\mathrm{O}) ;{ }^{1} \mathrm{H}$ NMR $(200 \mathrm{MHz}): 0.19(\mathrm{~s}, 9 \mathrm{H}), 1.09$ and $1.22(\mathrm{~s}$, $3 \mathrm{H}$ each, C-2- $\left.\mathrm{CH}_{3}{ }^{\prime} \mathrm{s}\right), 1.79\left(\mathrm{ddd}, 1 \mathrm{H}, J_{3 \alpha, 4 \beta}=2.6 \mathrm{~Hz}, J_{3 \alpha .4 \alpha}=\right.$ $\left.6.6 \mathrm{~Hz}, J_{\text {gem }}=14.2 \mathrm{~Hz}, \mathrm{H}-3 \alpha\right), 1.95\left(\mathrm{ddd}, 1 \mathrm{H}, J_{3 \beta, 4 \beta}=5.6 \mathrm{~Hz}\right.$, $J_{3 \beta, 4 \alpha}=12.5 \mathrm{~Hz}, J_{g e m}=14.2 \mathrm{~Hz}, \mathrm{H}-3 \beta$ ), 2.43 (ddd, $1 \mathrm{H}, J_{4 \beta, 3 \alpha}=$ $2.6 \mathrm{~Hz}, J_{4 \beta, 3 \beta}=5.6 \mathrm{~Hz}, J_{g e m}=19.1 \mathrm{~Hz}, \mathrm{H}-4 \beta$ ), 2.63 (ddd, $1 \mathrm{H}$, $\left.\mathrm{J}_{4 \alpha, 3 \alpha}=6.6 \mathrm{~Hz}, J_{4 \alpha, 3 \beta}=12.5 \mathrm{~Hz}, J_{g e m}=19.1 \mathrm{~Hz}, \mathrm{H}-4 \alpha\right), 5.60(\mathrm{~d}$, $\left.1 \mathrm{H}, J_{8,9}=1.9 \mathrm{~Hz}, \mathrm{H}-8\right), 6.98\left(\mathrm{dd}, 1 \mathrm{H}, J_{9,8}=1.9 \mathrm{~Hz}, J_{9,10}=\right.$ $5.4 \mathrm{~Hz}, \mathrm{H}-9), 7.05\left(\mathrm{~d}, 1 \mathrm{H}, J_{10.9}=5.4 \mathrm{~Hz}, \mathrm{H}-10\right) ;{ }^{13} \mathrm{C} \mathrm{NMR}$ $(50 \mathrm{MHz}):-2.15(\mathrm{q}), 25.76(\mathrm{q}), 22.29(\mathrm{q}), 31.63(\mathrm{~s}, \mathrm{C}-2), 34.20$ and 36.16(t, C-3 and C-4), 86.48(d, C-8), 99.87(s, C-1), 144.16 and 144.44(d, C-9 and C-10), 160.77 and 174.23 (s, C-6 and C-7), 195.64(s, C-5); mass spectrum: 262(15, $\left.\mathrm{M}^{*+}\right), 247(50, \mathrm{M}-\mathrm{Me})$, $109\left(100, \mathrm{M}-\mathrm{C}_{8} \mathrm{H}_{16} \mathrm{OSi}\right)$. Exact Mass calcd. for $\mathrm{C}_{15} \mathrm{H}_{22} \mathrm{O}_{2} \mathrm{Si}$ : 262.1389; found: 262.1376 .

\section{2,2,7-Trimethyl-11-oxatricyclo[6.2,1.0 $\left.0^{1,6}\right]$ undec-6,9-dien-5-one}

(66)

General procedure 5 was used for the Lewis acid-mediated IMDAF reaction of compound 17. Thus, enone $17(99.1 \mathrm{mg}$, $0.485 \mathrm{mmol})$ was treated with $\mathrm{MeAlCl}_{2}(553 \mu \mathrm{L}, 0.553 \mathrm{mmol})$ at $-60^{\circ} \mathrm{C}$ for $0.5 \mathrm{~h}$ to provide adduct $66(98.5 \mathrm{mg}, 0.482 \mathrm{mmol})$ in 
$99 \%$ recovery as a white, crystalline solid, which could not be purified by flash chromatography without isomerization of the C-6, C-7 double bond; IR (neat) $\mathrm{cm}^{-1}: 1666(\mathrm{C}=\mathrm{O}) ;{ }^{1} \mathrm{H}$ NMR $(200 \mathrm{MHz}): 1.10$ and 1.21 (s, $3 \mathrm{H}$ each), 1.75 (ddd, $1 \mathrm{H}, J_{3 \alpha, 4 \beta}=$ $\left.3.0 \mathrm{~Hz}, J_{3 \alpha, 4 \alpha}=6.7 \mathrm{~Hz}, J_{\mathrm{gem}}=12.9 \mathrm{~Hz}, \mathrm{H}-3 \alpha\right), 1.89(\mathrm{dt}, 1 \mathrm{H}$, $\left.J_{3 \beta, 4 \beta}=3.0 \mathrm{~Hz}, J_{3 \beta .4 \%}=J_{g e m}=12.9 \mathrm{~Hz}, \mathrm{H}-3 \beta\right), 2.31(\mathrm{~s}, 3 \mathrm{H}$, $\left.\mathrm{C}-8-\mathrm{CH}_{3}\right), 2.43\left(\mathrm{ddd}, 1 \mathrm{H}, J_{4 \beta .3 \alpha}=3.0 \mathrm{~Hz}, J_{4 \beta .3 \beta}=3.0 \mathrm{~Hz}, J_{g^{\mathrm{t}} \mathrm{m}}=\right.$ $16.3 \mathrm{~Hz}, \mathrm{H}-4 \beta), 2.50-2.75(\mathrm{~m}, 1 \mathrm{H}, \mathrm{H}-4 \alpha), 5.10\left(\mathrm{~d}, 1 \mathrm{H}, J_{8.9}=\right.$ $1.8 \mathrm{~Hz}, \mathrm{H}-8), 7.04\left(\mathrm{dd}, 1 \mathrm{H}, J_{9,8}=1.8 \mathrm{~Hz}, J_{9,10}=5.4 \mathrm{~Hz}, \mathrm{H}-9\right.$ ), $7.09\left(\mathrm{~d}, 1 \mathrm{H}, J_{10.9}=5.4 \mathrm{~Hz}, \mathrm{H}-10\right) ;{ }^{13} \mathrm{C}$ NMR $(50 \mathrm{MHz}): 15.63(\mathrm{q})$, 22.02(q), 25.44(q), 31.64(s, C-2), 34.20(t), 36.16(t), 86.12(d, C-8), 99.28(s, C-1), 142.04 and $145.57(\mathrm{~d}, \mathrm{C}-9$ and C-10), $171.98(\mathrm{~s}, \mathrm{C}-6$ and $\mathrm{C}-7), 196.32(\mathrm{~s}, \mathrm{C}-5)$; mass spectrum: 204(14, $\left.\mathrm{M}^{+}\right), \quad$ 163(24, $\left.\mathrm{M}-\mathrm{HC} \equiv \mathrm{CH}\right), \quad 109(100, \mathrm{M}-$ $\left.\left(\mathrm{CH}_{2}\right)_{2} \mathrm{C}(\mathrm{O}) \mathrm{C} \equiv \mathrm{CCH}_{3}\right)$. Exact Mass calcd. for $\mathrm{C}_{13} \mathrm{H}_{16} \mathrm{O}_{2}:$ 204.1 150; found: 204.1146.

\section{Acknowledgements}

We thank the Natural Sciences and Engineering Research Council of Canada (NSERC), the University of Calgary, and the University of Windsor for financial support, and NSERC for a post-graduate scholarship (to C.R.).

1. (a) G. Brieger and J. N. Bennett. Chem. Rev. 80, 63 (1980); (b) R. Sustman and J. Sauer. Angew. Chem. Int. Ed. Engl. 19, 779 (1980); (c) A. G. Fallis. Can. J. Chem. 62, 183 (1984); (d) E. Ciganek. Org. React. 32, 1 (1984); (e) D. F. Taber. Intramolecular Diels-Alder reactions and Alder ene reactions. Springer-Verlag, New York. 1984; $(f)$ K. Hayakawa and K. Kanematsu. J. Synth. Org. Chem. Jpn. 44, 109 (1986); (g) D. Craig. Chem. Soc. Rev. 16, 187 (1987).

2. S. Cauwberghs, P. J. De Clercq, B. Tinant, and J. P. Declercq. Tetrahedron Lett. 29, 2493 (1988); T. Ghosh and H. Hart. J. Org. Chem. 54, 5073 (1989); A. Padwa, Y. S. Kulkarni, and L. W. Terry. J. Org. Chem. 55, 2478 (1990); A. P. Kozikowski and W. Tuckmantel. J. Org. Chem. 56, 2826 (1991); K. Fischer and S. Hunig. J. Org. Chem. 52, 564 (1987); B. L. Feringa, O. J. Gelling, and L. Meesters. Tetrahedron Lett. 31, 7201 (1990); W. M. Grootaert and P. J. De Clercq. Tetrahedron Lett. 27, 1731 (1986); F. Nuyttens, G. Appendino, and P. J. De Clercq. Synlett, 526 (1991).

3. (a) J. Moursounidis and D. Wede. Tetrahedron Lett. 27, 3045 (1986); (b) W. M. Best and D. Wege. Aust. J. Chem. 39, 635 (1986); (c) Aust. J. Chem. 39, 647 (1986); (d) Y. Yamaguchi, J. Yamada, K. Hayakawa, and K. Kanematsu. J. Org. Chem. 52, 2040 (1987); (e) H. Finch, L. M. Harwood, G. M. Robertson, and R. C. Sewell. Tetrahedron Lett. 30, 2585 (1989); $(f)$ K. H. Dotz, R. Noack, K. Harms, and G. Muller. Tetrahedron, 46, 1235 (1990); $(g)$ M. E. Jung. Synlett, 1, 186 (1990); (h) M. E. Jung and J. Gervay. J. Am. Chem. Soc. 113, 224 (1991); (i) W. Blokzijl, M. J. Blandamer, and J. B. F. N. Engberts. J. Am. Chem. Soc. 113, 4241 (1991).

4. (a) P. J. De Clercq and L. A. Van Royen. Synth. Commun. 9, 771 (1979); (b) L. M. Harwood, S. A. Leeming, N. S. Isaacs, G. Jones, J. Pickard, R. M. Thomas, and D. A. Watkin. Tetrahedron Lett. 29, 5017 (1988); (c) L. M. Harwood, G. Jones, J. Pickard, R. M. Thomas, and D. Watkin. Tetrahedron Lett. 29, 5825 (1988); (d) L. A. Van Royen, R. Mijngheer, and P. J. De Clercq. Tetrahedron Lett. 23, 3283 (1982); (e) Tetrahedron Lett. 24, 3145 (1983); (f) Bull. Soc. Chim. Belg. 93, 1019 (1984); (g) Tetrahedron, 41, 4667 (1985); (h) B. A. Keay. J. Chem. Soc. Chem. Commun. 419 (1987); (i) P. Missiaen and P. J. De Clercq. Bull. Soc. Chim. Belg. 96, $105(1987) ;(j)$ P. W. Dibble and B. A. Keay. Tetrahedron Lett. 30, 1045 (1989); (k) C. Rogers and B. A. Keay. Tetrahedron Lett. 30, 1349 (1989); (l) P. Missiaen and P. J. De Clercq. Bull. Soc. Chim. Belg. 99, 271 (1990); (m) G. Appendino, J. Hoflack, P. J.
De Clercq, G. Chiari, and M. Calleri. Tetrahedron, 44, 4605 (1988); (n) S. G. Cawberghs and P. J. De Clercq. Tetrahedron Lett. 29, 6501 (1988).

5. (a) S. J. Burrell, A. E. Derome, M. S. Edenborough, L. M. Harwood, S. A. Leeming, and N. S. Isaacs. Tetrahedron Lett. 26, 2229 (1985); (b) L. M. Harwood, G. Jones, J. Pickard, R. M. Thomas, and D. Watkin. J. Chem. Soc. Chem. Commun. 605 and 608 (1990); (c) L. M. Harwood, T. Ishikawa, H. Phillips, and D. Watkin. J. Chem. Soc. Chem. Commun. 527 (1991).

6. D. D. Sternbach and D. M. Rossana. J. Am. Chem. Soc. 104, 5853 (1982).

7. D. D. Sternbach and D. M. Rossana. Tetrahedron Lett. 23, 303 (1982).

8. M. E. Jung and J. Gervay. J. Am. Chem. Soc. 111, 5469 (1989); Tetrahedron Lett. 29, 2429 (1988); D. D. Sternbach and D. M. Rossana. Tetrahedron Lett. 26, 591 (1985); J. Org. Chem. 49, 3428 (1984).

9. (a) B. H. Lipshutz. Chem. Rev. 86, 795 (1986); (b) W. A. Nugent, R. J. McKinney, and R. L. Harlow, Organometallics, 3, 1315 (1984); (c) F. Brion. Tetrahedron Lett. 23, 5299 (1982); (d) H. Kotsuki, K. Asao, and H. Ohnishi. Bull. Soc. Chem. Jpn. 3339 (1984); (e) E. Vieira and P. Vogel. Helv. Chim. Acta, 65, 1700 (1982); $(f)$ J. A. Moore and E. M. Partain. J. Org. Chem. 48, 1105 (1983).

10. (a) D. A. Smith and K. N. Houk. Tetrahedron Lett. 32, 1549 (1991); (b) K. Takeda, T. Kobayashi, K. Saito, and E. Yoshii. J. Org. Chem. 53, 1092 (1988); (c) W. R. Roush and R. Riva. J. Org. Chem. 53, 710 (1988); (d) W. R. Roush, A. P. Essenfeld, and J. S. Warmus. Tetrahedron Lett. 28, 2447 (1987); (e) J. A. Marshall. B. G. Shearer, and S. L. Crooks. J. Org. Chem. 52, 1236 (1987); $(f)$ J. A. Marshall, J. Grote, and J. E. Audia. J. Am. Chem. Soc. 109, 1186 (1987); $(g)$ D. A. Smith, K. Sukan, and K. N. Houk. Tetrahedron Lett. 27, 4877 (1986); (h) J. A. Marshall, J. E. Audia, J. Grote, and B. G. Shearer. Tetrahedron, 42, 2893 (1986); (i) J. A. Marshall, J. E. Audia, and J. Grote. J. Org. Chem. 49, 5279 (1984); $(j)$ K. Sakan and D. A. Smith. Tetrahedron Lett. 25, 2081 (1984); (k) K. J. Shea and J. W. Gilman. Tetrahedron Lett. 24, 657 (1983); (l) W. R. Roush and H. R. Gillis. J. Org. Chem. 47, 4825 (1982); (m) W. R. Roush, H. R. Gillis, and A. I. Ko. J. Am. Chem. Soc. 104, 2269 (1982); $(n)$ W. R. Roush and S. E. Hall. J. Am. Chem. Soc. 103, 5200 (1981).

11. K. A. Parker and M. R. Adamchuk. Tetrahedron Lett. 1689 (1978).

12. T. Takebayashi, N. Iwasawa, and T. Mukaiyama. Bull. Chem. Soc. Jpn. 56, 1107 (1983); T. Mukaiyama and T. Takebayashi. Chem. Lett. 1013 (1980); T. Mukaiyama, T. Tsuji, and N. Iwasawa. Chem. Lett. 697 (1979).

13. B. L. Feringa, O. J. Gelling, and L. Meesters. Tetrahedron Lett. 31, 7201 (1990).

14. P. Bosshard and C. H. Eugster. Adv. Heterocycl. Chem. 7, 378 (1966); F. M. Dean. Adv. Heterocycl. Chem. 30, 168 (1982); 31, 238 (1982).

15. B. A. Keay, D. Rajapaksa, and R. Rodrigo. Can. J. Chem. 62, 1093 (1984).

16. C. Rogers and B. A. Keay. Tetrahedron Lett. 32, 6477 (1991); Synlett, 353 (1991).

17. B. J. Wakefield. Organolithium methods. Academic Press, Toronto. 1988.

18. E.-I. Negishi, L. D. Boardman, H. Sawada, V. Bagheri, A. T. Stoll, J. M. Tour, and C. L. Rand. J. Am. Chem. Soc. 110, 5383 (1988).

19. W. F. Bailey, T. T. Nurmi, J. J. Patricia, and W. Wang. J. Am. Chem. Soc. 109, 2442 (1987).

20. A. J. Mancuso and D. Swern. Synthesis, 165 (1981).

21. Z. M. Ismail and H. M. R. Hoffmann. Angew. Chem. Suppl. 985 (1983). 
22. J. F. Normant and C. Piechuk. Bull. Soc. Chim. Fr. 2402 (1972).

23. (a) J. S. Ng, J. R. Behling, A. L. Campbell, D. Nguyen, and B. Lipshutz. Tetrahedron Lett. 29, 3045 (1988); (b) K. Mori and M. Matsui. Tetrahedron, 22, 2883 (1966).

24. T. K. Jones and S. E. Denmark. Org. Synth. 64, 182 (1985); G. Piancatteli, A. Sattri, and M. D'Auria. Synthesis, 245 (1982).

25. L. Brandsma. Preparative acetylene chemistry. Elsevier, Amsterdam. 1971.

26. B. B. Snider, D. J. Rodini, M. K. Karras, T. C. Kirk, E. A. Deutsch, R. Cordova, and R. T. Price. Tetrahedron, 37, 3927 (1981).

27. H. Kotsuki. Bull. Chem. Soc. Jpn. 57, 3339 (1984).

28. (a) M. Kakushima, J. Espinosa, and Z. Valenta. Can. J. Chem. 54, 3304 (1976); (b) W. Oppolzer, C. Chapuis, G. M. Dao, D. Reichlin, and T. Godel. Tetrahedron Lett. 23, 4781 (1982); (c) K. Sakan and B. A. Craven. J. Am. Chem. Soc. 105, 3732 (1983).

29. W. C. Still, M. Kahn, and A. Mitra. J. Org. Chem. 43, 2923 (1978).

30. R. M. Beesley, C. K. Ingold, and J. F. Thorpe. J. Chem. Soc. 107, 1080 (1915); C. K. Ingold. J. Chem. Soc. 119, 305 (1921); N. L. Allinger and V. Zalkow. J. Org. Chem. 25, 701
(1960); T. C. Bruice and U. K. Pandit. J. Am. Chem. Soc. 82, 5858 (1960).

31. W. R. Roush and H. R. Gillis. J. Org. Chem, 45, 4267 (1980).

32. B. P. Susz. Bull. Soc. Chem. Fr. 2671 (1965); T.-L. Ho. Chem. Rev. 75, 1 (1975); J. March. Advanced organic chemistry. 3rd ed. Wiley and Sons, New York. 1985. pp. 218223.

33. S. J. Hecker and C. H. Heathcock, J. Org. Chem. 50, 5159 (1985); S. E. Hall and W. R. Roush. J. Org. Chem. 47, 4611 (1982); W. R. Roush and S. M. Peseckis. J. Am. Chem. Soc. 103, 6696 (1981); P. A. Jacobi and D. G. Walker. J. Am. Chem. Soc. 103, 4611 (1981); P. A. Jacobi, D. G. Walker, and I. M. A. Odeh. J. Org. Chem. 46, 2065 (1981); G. A. Kraus. J. Am. Chem. Soc. 102, 1974 (1980); L. H. Klemm, T. M. McGuire, and K. W. Gopinath. J. Org. Chem. 41, 2571 (1976).

34. B. A. Keay, H. P. Plaumann, D. Rajapaksa, and R. Rodrigo. Can. J. Chem. 61, 1987 (1983); R. M. Silverstein, G. C. Bassler, and T. C. Morrill. Spectrometric identification of organic compounds. 5th ed. Wiley and Sons, Toronto. 1991.

35. T. Mukayama and T. Takebayashi, Chem. Lett. 1013 (1980).

36. I. Fleming. Frontier orbitals and organic chemical reactions. Wiley and Sons, New York. 1976. pp. 86-181.

37. D. D. Perrin and W. L. F. Armarego. Purification of laboratory chemicals. 3rd ed. Pergamon Press, Toronto. 1988. 